Miguel Fernando Montoya Vallejo

\title{
Algoritmo de Tomografia por Impedância Elétrica utilizando Programação Linear como Método de Busca da Imagem
}

Dissertação apresentada à Escola Politécnica da Universidade de São Paulo para obtenção do Título de Mestre em Engenharia. 
Miguel Fernando Montoya Vallejo

\section{Algoritmo de Tomografia por Impedância Elétrica utilizando Programação Linear como Método de Busca da Imagem}

Dissertação apresentada à Escola Politécnica da Universidade de São Paulo para obtenção do Título de Mestre em Engenharia.

Área de concentração:

Engenharia Mecânica

Orientador:

Prof. Dr. Raul Gonzalez Lima 


\section{Ficha Catalográfica}

Montoya Vallejo, Miguel Fernando

Algoritmo de Tomografia por Impedância Elétrica utilizando Programação Linear como Método de Busca da Imagem. São Paulo, 2007. $95 \mathrm{p}$.

Dissertação (Mestrado) - Escola Politécnica da Universidade de São Paulo. Departamento de Engenharia Mecânica.

1. Programação Linear. 2. Tomografia 3. Método dos Elementos Finitos 4. Otimização não-linear I. Universidade de São Paulo. Escola Politécnica. Departamento de Engenharia Mecânica Departamento de Engenharia Mecânica. II. t. 
A meus pais, Juan Carlos e Hilda Maria, obrigado por não poupar esforços em nossa educação 


\section{Agradecimentos}

A culminação de um trabalho é o resultado de um esforço conjunto de pessoas que contribuem com ideais e conhecimento. Quero agradecer

Ao amigo e Prof. Dr. Raul Gonzalez Lima, pelo apoio, paciência e confiança depositada.

À CNPq (Conselho Nacional de Desenvolvimento Científico e Tecnológico) pelo apoio financeiro deste trabalho.

Ao amigo Dr. Julio Cesar Ceballos Aya, pelo conhecimento transferido, paciência e sugestões que enriqueceram este trabalho.

A minha namorada, ponto de apoio e segurança nos momentos difíceis da vida.

A meu futuro sobrinho, olhar o futuro é enxergar a consequência do presente

A meu irmão e amigo Eng. Juan Felipe Montoya Vallejo, pelo carinho e apoio na iniciativa de continuar com meus estudos.

Ao amigo Eng. Fernando Silva de Moura com quem tenho a satisfação de trabalhar todos os dias.

A todos meus familiares, aqueles que ainda tenho e aqueles que já não estão, pelo apoio e carinho.

A todos os amigos que, de uma maneira ou de outra, contribuíram para a realização do trabalho. 


\section{Resumo}

A Tomografia por Impedância elétrica (TIE) tem como objetivo gerar imagens da distribuição de resistividade dentro de um domínio. A TIE injeta correntes em eletrodos alocados na fronteira do domínio e mede potenciais elétricos através dos mesmos eletrodos. A TIE é considerada um problema inverso, não-linear e mal posto. Atualmente, para gerar uma solução do problema inverso, existem duas classes de algoritmos para estimar a distribuição de resistividade no interior do domínio, os que estimam variações da distribuição de resistividade do domínio e os absolutos, que estimam a distribuição de resistividade. Variações da distribuição de resistividade são o resultado da solução de um sistema linear do tipo $A x=b$. O objetivo do presente trabalho é avaliar o desempenho da Programação Linear (PL) na solução do sistema linear, avaliar o algoritmo quanto a propagação de erros numéricos e avaliar os efeitos de restringir o espaço solução através de restrições de PL. Os efeitos do uso de Programação Linear é avaliado tanto em métodos que geram imagens de diferenças, como o Matriz de Sensibilidade, como em métodos absolutos, como o Gauss-Newton. Mostra-se neste trabalho que o uso da PL diminui o erro numérico propagado quando comparado ao uso do algoritmo LU Decomposition. Resulta também que reduzir o espaço solução, diretamente através de restrições de PL, melhora a resolução em resistividade e a resolução espacial da imagem quando comparado com o uso de LU Decomposition.

palavras-chave: Tomografia por Impedância Elétrica, Programação Linear, Matriz de Sensibilidade, Gauss-Newton. 


\section{Abstract}

Electrical impedance tomography (EIT) generates images of the resistivity distribution of a domain. The EIT method inject currents through electrodes placed on the boundary of the domain and measures electric potentials through the same electrodes. EIT is considered an inverse problem, non-linear and ill-conditioned. There are two classes of algorithms to estimate the resistivity distribution inside the domain, difference images algorithms, which estimate resistivity distribution variations, and absolute images algorithms, which estimate the resistivity distribution. Resistivity distribution variations are the solution of a linear system, say $A x=b$. In this work, the main objective is to evaluate the performance of Linear Programming (LP) solving an EIT linear system from the point of view of the numerical error propagation and the ability to constrain the solution space. The impact of using LP to solve an EIT linear system is evaluated on a difference image algorithm and on an absolute algorithm. This work shows that the use of LP diminishes the numerical error propagation compared to LU Decomposition. It is also shown that constraining the solution space through LP improves the resistivity resolution and the spatial resolution of the images when compared to LU Decomposition.

key-word: Electrical Impedance Tomography, Linear Programming, Sensitivity Matrix, Gauss-Newton. 


\title{
Sumário
}

\section{Lista de Figuras}

\author{
Lista de Tabelas
}

\section{Lista de Abreviaturas}

\section{Convenções e Lista de Símbolos}

1 Introdução 1

2 Objetivos 3

3 Revisão Bibliográfica $\quad 4$

3.1 Formulação Teórica . . . . . . . . . . . . . . . . 7

3.1.1 Formulação Teórica do Domínio . . . . . . . . . . . 7

3.1.2 Método de Elementos Finitos . . . . . . . . . . . . . . 9

3.1.3 Modelo de Eletrodo 2D . . . . . . . . . . . . . . . . . . . 11

3.1.4 Padrões de Injeção de Corrente e de Medida de Potencial . 12

3.1.5 Solução do Problema Direto . . . . . . . . . . . . . . . . 14

3.1.6 Solução do Problema Inverso . . . . . . . . . . . . . . . . . 14

3.1.7 Restrição de Inclinação Máxima na Distribuição de Resistividade $(\mathrm{RIM}) \ldots \ldots \ldots \ldots$

3.2 Programação Linear . . . . . . . . . . . . . . . . . . . . . . 17

3.2.1 Definição de Programação Linear . . . . . . . . . . . . . 17

3.2 .2 Aplicações Industriais . . . . . . . . . . . . . . . . . . 18

3.2 .3 Formulação Matemática . . . . . . . . . . . . . . . 18 
3.2.4 Como Utilizar PL na TIE . . . . . . . . . . . . . . . . . 19

3.3 Programação Linear Seqüencial (PLS) . . . . . . . . . . . . 20

3.3.1 Limites Móveis . . . . . . . . . . . . . . . . 21

3.4 O Algoritmo Simplex . . . . . . . . . . . . . . . . 23

3.4.1 Passo 1. Solução Básica Viável Inicial $x \ldots \ldots 25$

3.4.2 Passo 2. É Possível uma Solução Melhor ? Em Caso Afirmativo, Escolher Nova Variável Básica $x \ldots . . . . .25$

3.4.3 Passo 3. Atribuição de Valor à Nova Variável Básica; Determinação da Nova Variável não Básica . . . . . . . . 26

3.5 Matriz de Sensibilidade . . . . . . . . . . . . . . 28

3.5.1 Abordagem do Problema de Imagens de Variação de Resistividade Utilizando Matriz de Sensibilidade . . . . . . . . 29

3.6 Método Newton-Raphson . . . . . . . . . . . . . . . . . . . . 30

3.6.1 Método Gauss-Newton . . . . . . . . . . . . . . . . . 32

3.6.2 Abordagem do Problema de Imagens Absolutas de Resistividade Utilizando Método de Gauss-Newton . . . . . . . . 32

3.6.3 Implementação da RIM na Distribuição de Resistividade . 34

4 Metodologia $\quad 35$

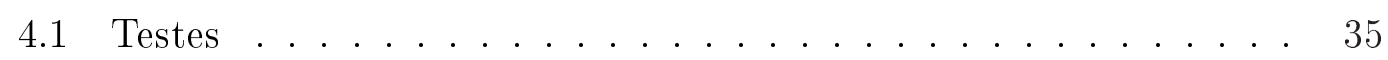

4.1.1 Diminuição do Erro Numérico via PL . . . . . . . . . . . . 38

4.1.2 PL Comparado com LU Decomposition - Matriz de Sensibilidade .................. . . 40

4.1.3 PL comparado com LU Decomposition - Gauss-Newton . . 40

4.1.4 Dados de Simulação Numérica . . . . . . . . . . . . . . . . 40

4.1.5 Dados da Bancada Experimental . . . . . . . . . . . . . 44

$\begin{array}{llr}5 & \text { Resultados } & 48\end{array}$

5.1 Resultados Obtidos com PL na Diminuição do Erro Numérico . . 48 
5.2 Resultados Implementando PL e LU Decomposition no Problema de Imagens de Variação de Resistividade . . . . . . . . . . . . . . 51

5.2 .1 Dados de Simulação Numérica . . . . . . . . . . . . . . 51

5.2.2 Dados da Bancada Experimental . . . . . . . . . . 57

5.3 Resultados Implementando PLS e LU Decomposition no Método Gauss-Newton . . . . . . . . . . . . . . . 63

5.3.1 Dados de Simulação Numérica . . . . . . . . . . . . 63

5.4 Comparação das Imagens Obtidas com LU Decomposition e PLS Utilizando RIM . . . . . . . . . . . . . . . . 70

5.4.1 Dados de Simulação Numérica . . . . . . . . . . . . 70

5.4.2 Dados de Bancada Experimental . . . . . . . . . 76

5.4.3 Dados do Tórax Humano . . . . . . . . . . . . . . . 84

6 Análise dos Resultados $\quad 86$

6.1 PL na Diminuição do Erro Numérico . . . . . . . . . . . 86

6.2 Comparação dos Resultados Obtidos com PL e LU Decomposition no Problema de Imagens de Variação de $\rho \ldots$. . . . . . . . . 86

6.2.1 Dados de Simulação Numérica . . . . . . . . . . . . 86

6.2.2 Dados da Bancada Experimental . . . . . . . . . . 87

6.3 Comparação dos Resultados Obtidos com LU Decomposition e PLS sem RIM . . . . . . . . . . . . . . . . . . . . . 87

6.3.1 Dados de Simulação Numérica . . . . . . . . . . . . . 87

6.4 Comparação dos Resultados Obtidos com LU Decomposition e PLS e RIM ........................... 88

6.4.1 Dados de Simulação Numérica . . . . . . . . . . . . 88

6.4.2 Dados da Bancada Experimental . . . . . . . . . . 88

6.4.3 Dados do Tórax Humano . . . . . . . . . . . . . . . . . . 89 


\section{Lista de Figuras}

3.1 Seção Transversal do tórax (imagem modificada para visualizar os órgãos do tórax $) \ldots \ldots \ldots$. . . . . . . . . . . . . . . . . . 9

3.2 Discretização do tórax em elementos triangulares (BORSIC et al., $2000) \ldots \ldots \ldots \ldots \ldots \ldots$

3.3 Discretização da interface eletrodo-pele . . . . . . . . . . . . . 11

3.4 Discretização do eletrodo em dois elementos e seis nós . . . . . . . 12

3.5 Padrão de corrente (a) pula um eletrodo (b) pula três eletrodos . 13

3.6 Determinação da circunferência de radio $\mathbf{R}$ dentro da qual buscamse os vizinhos do elemento escolhido . . . . . . . . . . . . . . . . 16

3.7 Procurando os vizinhos do elemento $i$ e determinando as distâncias aos elementos vizinhos . . . . . . . . . . . . . . . . 16

3.8 Conceituação de PL . . . . . . . . . . . . . . . . . . . . . . . . 18

3.9 Determinação dos limites móveis na iteração $k+1$ do método GaussNewton . . . . . . . . . . . . . . . . 23

3.10 Fluxograma do algoritmo Simplex (BREGALDA; OLIVEIRA; BORNS-

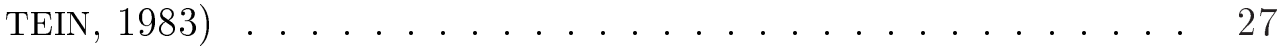

3.11 O ponto $x_{n+1}$ denota a aproximação do método Newton-Raphson ao zero da função $f(x) \ldots \ldots 31$

4.1 Objeto posicionado (a) na borda (b) no centro . . . . . . . 36

4.2 Objeto posicionado (a) na borda (b) no centro . . . . . . . . 37

4.3 Objetos de agar simulando a geometria do pulmão e coração . . 37

4.4 Posicionamento dos eletrodos para gerar imagens do tórax humano 38

4.5 Malha composta por 1938 nós, 606 elementos e 30 eletrodos . . . 40

4.6 Malhas de elementos finitos para resolver o problema (a) direto e (b) inverso . . . . . . . . . . . . . . . . . . . . . . . 4 41 
4.7 Malhas de elementos finitos para resolver o problema (a) direto e (b) inverso . . . . . . . . . . . . . . . . . . . . . . . . . 42

4.8 Malhas de elementos finitos para resolver o problema (a) direto e (b) inverso . . . . . . . . . . . . . . . . . . . . . . . . . 42

4.9 Malhas de elementos finitos em 2D para resolver o problema (a) direto e (b) inverso . . . . . . . . . . . . . . . . . . . . 43

4.10 Malhas de elementos finitos em 2D para resolver o problema (a) direto e (b) inverso . . . . . . . . . . . . . . . . 43

4.11 Malha de elementos finitos para trabalhar com dados da bancada experimental . . . . . . . . . . . . . . . . . . . . 44

4.12 Malha de elementos finitos para trabalhar com dados da bancada experimental . . . . . . . . . . . . . . . . .

4.13 Medidas para gerar a malha de elementos finitos (a) distância entre eletrodos e (b) largura do tórax . . . . . . . . . . . . . 46

4.14 Malha de elementos finitos para gerar imagens do tórax humano . 46

4.15 Tomógrafo por Impedância Elétrica . . . . . . . . . . . . . . . . 47

$5.1 \quad$ Autovalores para $\alpha=1,0 e^{-4} \ldots \ldots \ldots$. . . . . . . 48

5.2 Autovalores para $\alpha=1,0 e^{-5} \ldots \ldots \ldots$. . . . . . . . 49

5.3 Autovalores para $\alpha=1,0 e^{-6} \ldots \ldots \ldots \ldots$. . . . . . . . 49

5.4 Autovalores para $\alpha=1,0 e^{-7} \ldots \ldots \ldots . \ldots . \ldots 50$

5.5 Distribuição de resistividade, elemento perturbado na borda (a) com PL e (b) LU Decomposition . . . . . . . . . . . . . . . . . 51

5.6 Imagem interpolada (a) com PL e (b) LU Decomposition . . . . . 52

5.7 Distribuição de resistividade, elemento perturbado no centro (a) com PL e (b) LU Decomposition . . . . . . . . . . . . . . . . . 52

5.8 Imagem interpolada (a) com PL e (b) LU Decomposition . . . . . 52

5.9 Distribuição de resistividade, imagem interpolada, objeto na borda com dados experimentais utilizando PL . . . . . . . . . . . . . 53

5.10 Distribuição de resistividade, imagem interpolada, objeto na borda com dados experimentais utilizando LU Decomposition . . . . . . 53 
5.11 Distribuição de resistividade, objeto na borda com dados experimentais (a) com PL e (b) LU Decomposition . . . . . . . . . . . 54

5.12 Imagem interpolada (a) com PL e (b) LU Decomposition . . . . . 54

5.13 Imagem interpolada em perspectiva (a) com PL e (b) LU Decom-

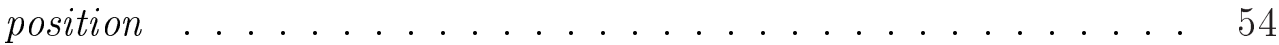

5.14 Distribuição de resistividade, objeto no centro com dados experimentais, solução via PL . . . . . . . . . . . . . . 55

5.15 Distribuição de resistividade, imagem interpolada, objeto no centro com dados experimentais, solução via LU Decomposition . . . . .

5.16 Distribuição de resistividade no centro com dados experimentais (a) com PL e (b) LU Decomposition . . . . . . . . . . . . . . . 56

5.17 Imagem interpolada (a) com PL e (b) LU Decomposition . . . . . 56

5.18 Imagem interpolada em perspectiva (a) com PL e (b) LU Decomposition ......................... 56

5.19 Obtenção de imagem de variação de resistividade na borda com dados experimentais utilizando PL . . . . . . . . . . . 57

5.20 Obtenção de imagem de variação de resistividade na borda com dados experimentais utilizando LU Decomposition . . . . . . . . 57

5.21 Obtenção de imagem de variação de resistividade na borda com dados experimentais utilizando PL . . . . . . . . . . . 58

5.22 Obtenção de imagem de variação de resistividade na borda com dados experimentais utilizando LU Decomposition . . . . . . . . 58

5.23 Obtenção da distribuição de resistividade na borda com dados experimentais (a) com PL e (b) LU Decomposition . . . . . . . . . . 59

5.24 Imagem interpolada (a) com PL e (b) LU Decomposition . . . . . 59

5.25 Imagem interpolada em perspectiva (a) com PL e (b) LU Decom-

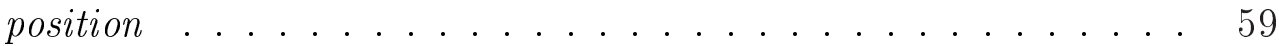

5.26 Obtenção de imagem de variação de resistividade na borda com dados experimentais utilizando PL . . . . . . . . . . . . . 60

5.27 Obtenção de imagem de variação de resistividade na borda com dados experimentais utilizando LU Decomposition . . . . . . . . . 
5.28 Obtenção de imagem de variação de resistividade na borda com dados experimentais utilizando PL . . . . . . . . . . . . 61

5.29 Obtenção de imagem de variação de resistividade na borda com dados experimentais utilizando LU Decomposition . . . . . . . . .

5.30 Distribuição de resistividade no centro com dados experimentais (a) com PL e (b) LU Decomposition . . . . . . . . . . . . . . . 62

5.31 Imagem interpolada (a) com PL e (b) LU Decomposition . . . . .

5.32 Imagem interpolada em perspectiva (a) com PL e (b) LU Decom-

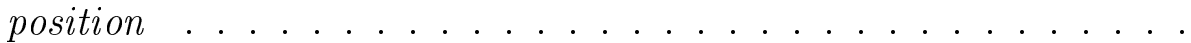

5.33 Obtenção de resistividade absoluta na borda com dados numéricos

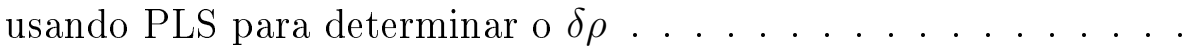

5.34 Obtenção de resistividade absoluta na borda com dados numéricos usando LU Decomposition para determinar o $\delta \rho \ldots \ldots$. . . . . . . 64

5.35 Índice de erro entre os potenciais elétricos medidos e os calculados 64

5.36 Obtenção de resistividade absoluta na borda com dados numéricos usando PLS para determinar o $\delta \rho \ldots \ldots . \ldots . \ldots . \ldots 65$

5.37 Obtenção de resistividade absoluta na borda com dados numéricos usando LU Decomposition para determinar o $\delta \rho \ldots \ldots . . . . .65$

5.38 Índice de erro entre os potenciais elétricos medidos e os calculados

5.39 Obtenção de resistividade absoluta na borda com dados numéricos usando PLS para determinar o $\delta \rho \ldots \ldots$. . . . . . . . 66

5.40 Obtenção de resistividade absoluta na borda com dados numéricos usando LU Decomposition para determinar o $\delta \rho \ldots$. . . . . . . . 66

5.41 Índice de erro entre os potenciais elétricos medidos e os calculados 66

5.42 Obtenção de resistividade absoluta no centro com dados numéricos usando PLS para determinar o $\delta \rho \ldots \ldots \ldots$. . . . . . . 67

5.43 Obtenção de resistividade absoluta no centro com dados numéricos usando LU Decomposition para determinar o $\delta \rho \ldots \ldots 7$

5.44 Índice de erro entre os potenciais elétricos medidos e os calculados 67

5.45 Obtenção de resistividade absoluta no centro com dados numéricos usando PLS para determinar o $\delta \rho \ldots \ldots . \ldots . \ldots 68$ 
5.46 Obtenção de resistividade absoluta no centro com dados numéricos usando LU Decomposition para determinar o $\delta \rho \ldots \ldots$. . . . . 68

5.47 Índice de erro entre os potenciais elétricos medidos e os calculados 68

5.48 Obtenção de resistividade absoluta no centro com dados numéricos usando PLS para determinar o $\delta \rho \ldots \ldots \ldots$. . . . . .

5.49 Obtenção de resistividade absoluta no centro com dados numéricos usando LU Decomposition para determinar o $\delta \rho$. . . . . . . . .

5.50 Índice de erro entre os potenciais elétricos medidos e os calculados

5.51 Obtenção de resistividade absoluta na borda com dados numéricos usando PLS e RIM para determinar o $\delta \rho \ldots$. . . . . . . . . 70

5.52 Obtenção de resistividade absoluta no centro com dados numéricos

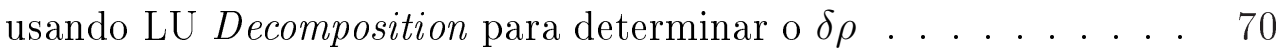

5.53 Índice de erro entre os potenciais elétricos medidos e os calculados 71

5.54 Obtenção de resistividade absoluta na borda com dados numéricos

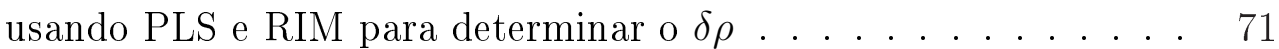

5.55 Obtenção de resistividade absoluta no centro com dados numéricos usando LU Decomposition para determinar o $\delta \rho \ldots . . . . . .77$

5.56 Índice de erro entre os potenciais elétricos medidos e os calculados 72

5.57 Obtenção de resistividade absoluta na borda com dados numéricos usando PLS e RIM para determinar o $\delta \rho \ldots$. . . . . . . . . 72

5.58 Obtenção de resistividade absoluta no centro com dados numéricos usando LU Decomposition para determinar o $\delta \rho \ldots . . . . . .72$

5.59 Índice de erro entre os potenciais elétricos medidos e os calculados 73

5.60 Obtenção de resistividade absoluta no centro com dados numéricos usando PLS e RIM para determinar o $\delta \rho \ldots . . . . . . . .773$

5.61 Obtenção de resistividade absoluta no centro com dados numéricos usando LU Decomposition para determinar o $\delta \rho \ldots . . . . . .73$

5.62 Índice de erro entre os potenciais elétricos medidos e os calculados 74

5.63 Obtenção de resistividade absoluta no centro com dados numéricos usando PLS e RIM para determinar o $\delta \rho \ldots$. . . . . . . . . . 74 
5.64 Obtenção de resistividade absoluta no centro com dados numéricos usando LU Decomposition para determinar o $\delta \rho \ldots \ldots . . . . .74$

5.65 Índice de erro entre os potenciais elétricos medidos e os calculados 75

5.66 Obtenção de resistividade absoluta no centro com dados numéricos usando PLS e RIM para determinar o $\delta \rho \ldots \ldots . . . . . .75$

5.67 Obtenção de resistividade absoluta no centro com dados numéricos usando LU Decomposition para determinar o $\delta \rho \ldots . . . . . .75$

5.68 Índice de erro entre os potenciais elétricos medidos e os calculados 76

5.69 Obtenção de resistividade absoluta na borda com dados experi-

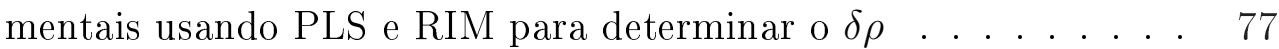

5.70 Obtenção de resistividade absoluta na borda com dados experimentais usando LU Decomposition para determinar o $\delta \rho \ldots$. . . 77

5.71 Índice de erro entre os potenciais elétricos medidos e os calculados 77

5.72 Obtenção de resistividade absoluta na borda com dados experimentais usando PLS e RIM para determinar o $\delta \rho \ldots \ldots . . .78$

5.73 Obtenção de resistividade absoluta na borda com dados experimentais usando LU Decomposition para determinar o $\delta \rho \ldots \ldots$

5.74 Índice de erro entre os potenciais elétricos medidos e os calculados 78

5.75 Obtenção de resistividade absoluta na borda com dados experimentais usando PLS e RIM para determinar o $\delta \rho \ldots$. . . . . . 79

5.76 Obtenção de resistividade absoluta na borda com dados experimentais usando LU Decomposition para determinar o $\delta \rho \ldots . . . .79$

5.77 Índice de erro entre os potenciais elétricos medidos e os calculados 79

5.78 Obtenção de resistividade absoluta no centro com dados experimentais usando PLS e RIM para determinar o $\delta \rho \ldots$. . . . . 80

5.79 Obtenção de resistividade absoluta no centro com dados experimentais usando LU Decomposition para determinar o $\delta \rho \ldots$. . . 80

5.80 Índice de erro entre os potenciais elétricos medidos e os calculados 80

5.81 Obtenção de resistividade absoluta no centro com dados experimentais usando PLS e RIM para determinar o $\delta \rho \ldots$. . . . . . 81 
5.82 Obtenção de resistividade absoluta no centro com dados experimentais usando LU Decomposition para determinar o $\delta \rho \ldots$. . . 81

5.83 Índice de erro entre os potenciais elétricos medidos e os calculados 81

5.84 Obtenção de resistividade absoluta no centro com dados experimentais usando PLS e RIM para determinar o $\delta \rho \ldots \ldots$. . . . . 82

5.85 Obtenção de resistividade absoluta no centro com dados experimentais usando LU Decomposition para determinar o $\delta \rho \ldots$. . . 82

5.86 Índice de erro entre os potenciais elétricos medidos e os calculados 82

5.87 Obtenção de resistividade absoluta com objetos de agar (a) com PLS e (b) LU Decomposition . . . . . . . . . . . . . . . . . . . . 83

5.88 Obtenção de resistividade absoluta com objetos de agar usando PLS e RIM para determinar o $\delta \rho \ldots \ldots$. . . . . . . . . . . 83

5.89 Obtenção de resistividade absoluta com objetos de agar usando

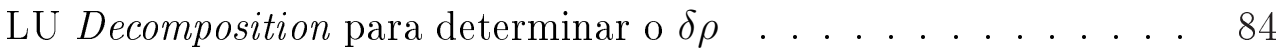

5.90 Obtenção de resistividade absoluta no tórax humano (a) com PLS e (b) LU Decomposition . . . . . . . . . . . . . . . . . . . . . . . 84

5.91 Obtenção de resistividade absoluta no tórax humano usando PLS e RIM para determinar o $\delta \rho \ldots \ldots \ldots \ldots$. . . . . . . . . 85

5.92 Obtenção de resistividade absoluta no tórax humano usando LU Decomposition para determinar o $\delta \rho \ldots \ldots . . . . . .$. 


\section{Lista de Tabelas}

3.1 Diminuição dos limites móveis . . . . . . . . . . . . . . . . . . 23

3.2 Representação das primeiras m equações e da função objetivo . . 25

3.3 Tabela final depois de realizar as operações de pivotamento . . . . 26

5.1 Os limites são determinados somando e subtraindo $20 \%$ respectivamente . . . . . . . . . . . . . . . 50

5.2 Os limites são determinados somando e subtraindo $30 \%$ respectivamente . . . . . . . . . . . . . . . . 51 


\section{Lista de Abreviaturas}

TIE Tomografia por Impedância Elétrica

PL Programação Linear

VB Variável Básica

VNB Variável não Básica

IP Índice de desempenho

MEF Método de Elementos Finitos

EDP Equações Diferenciais Parciais

RIM Restrição de inclinação máxima na distribuição de resistividade

PLS Programação Linear Seqüencial

Fo Função Objetivo

LS Limite Superior

LI Limite Inferior

Ra Número de Restrições Ativas 


\section{Convenções e Lista de Símbolos}

\begin{tabular}{|c|c|c|}
\hline símbolo & descrição & página \\
\hline$\Omega$ & Domínio fechado & 7 \\
\hline$\partial \Omega$ & Superficie de contorno & 7 \\
\hline$\sigma$ & Condutividade elétrica do material & 7 \\
\hline $\mathbf{E}$ & Campo elétrico & 8 \\
\hline B & Densidade de fluxo magnético & 8 \\
\hline$\Psi$ & Potencial escalar & 8 \\
\hline $\mathbf{J}$ & Densidade de corrente & 8 \\
\hline$e$ & Número de eletrodos & 8 \\
\hline$n$ & Versor normal à superficie & 8 \\
\hline$J_{i}$ & Densidade de corrente do $i$-ésimo eletrodo & 8 \\
\hline$\rho$ & Resistividade elétrica do material & 9 \\
\hline$m$ & Número de nós da malha de elementos finitos & 9 \\
\hline$n$ & $\begin{array}{l}\text { Número de elementos triangulares da malha de elemen- } \\
\text { tos finitos }\end{array}$ & 9 \\
\hline$v_{e}$ & Distribuição de potencial no $m$-ésimo elemento da malha & 10 \\
\hline$V(x, y)$ & Distribuição de potencial em um domínio discretizado & 10 \\
\hline$a, b, c$ & Coeficientes interpoladores & 10 \\
\hline $\mathbf{Y}_{i}^{l}$ & Matriz de rigidez local para o $i$-ésimo elemento & 10 \\
\hline $\mathbf{Y}$ & Matriz de rigidez global & 10 \\
\hline$\alpha_{j}$ & Funções de forma & 11 \\
\hline$t$ & Espessura da interface do eletrodo & 12 \\
\hline$a$ & Largura do eletrodo & 12 \\
\hline $\mathbf{Y}_{i}^{e}$ & Matriz de rigidez do eletrodo & 12 \\
\hline$v$ & Vetor de potenciais nos nós & 14 \\
\hline$I$ & Vetor da corrente injetada & 14 \\
\hline $\boldsymbol{R}$ & $\begin{array}{l}\text { Rádio da circunferência para determinar os elementos } \\
\text { vizinhos do elemento } i\end{array}$ & 15 \\
\hline$\mu$ & Valor de inclinação máxima & 15 \\
\hline $\overrightarrow{x_{j}}$ & Variáveis principais & 19 \\
\hline
\end{tabular}




\begin{tabular}{lll}
\hline símbolo & descrição & página \\
\hline$\tilde{a}_{i j}, \tilde{b}_{i}, \tilde{c}_{j}$ & Coeficientes técnicos & 19 \\
$\overrightarrow{x_{0}}$ & Vetor Arbitrário & 19 \\
$\epsilon$ & Escalar perto de zero & 20 \\
$\rho^{\text {sup }}$ & Limite superior absoluto & 21 \\
$\rho^{\text {inf }}$ & Limite superior absoluto & 21 \\
$\delta \rho_{k+1}^{\text {sup }}$ & Limite superior móvel & 21 \\
$\delta \rho_{k+1}^{\text {inf }}$ & Limite inferior móvel & 21 \\
$\tau$ & Valor inicial de partida & 22 \\
$\tilde{v}_{j}$ & Vetor de potenciais nos nós para cada padrão de corrente & 28 \\
$I_{j}$ & Padrão de corrente injetado & 28 \\
$\delta \rho_{k}$ & Passo do Método Gauss-Newton & 33 \\
\hline
\end{tabular}




\section{Introdução}

A Tomografia por Impedância Elétrica (TIE), pode monitorar de forma contínua e não invasiva os pulmões durante a ventilação artificial.

A TIE permite obter imagens aplicando corrente elétrica de baixa potência no objeto a caracterizar. Por meio desta técnica, pode-se estimar a distribuição de resistividade de uma região do corpo humano em cujo contorno são medidos potenciais elétricos.

A aplicação de corrente elétrica é realizada através de eletrodos distribuídos ao redor do tórax. A estimação de imagens em TIE é considerado um problema inverso, não-linear e mal condicionado. Os métodos numéricos podem ser classificados em iterativos ou não iterativos. Nos métodos não iterativos considera-se, na maior parte dos métodos, que a distribuição de potencial é uma função linear da distribuição de resistividade elétrica e, portanto, as imagens resultam da solução de um sistema linear ou da multiplicação por uma matriz. Alguns exemplos são o Backprojection e a Matriz de Sensibilidade (TRIGO, 2001).

No caso dos métodos iterativos, considera-se que realmente existe uma relação não-linear entre os potenciais elétricos e a resistividade elétrica e, freqüentemente, utilizam um índice de desempenho que deve ser minimizado com respeito à imagem. Alguns exemplos são o Método de Newton-Raphson (ARUCA, 2002), o Método da Otimização Topológica (LIMA; SILVA; LIMA, 2003) e variantes do Filtro de Kalman (TRIGO, 2001).

Acredita-se que algoritmos de TIE utilizando Programação Linear (PL) como método de busca da imagem teriam como principais vantagens comparados a outros algoritmos já desenvolvidos a capacidade de propagar pouco ruído numérico e a facilidade de restringir o espaço de solução através de restrições de Programação Linear. Há diversos trabalhos que sugerem que a PL se comporta bem com o problema de TIE (LIMA; SILVA; LIMA, 2003; LIMA, 2006; MELLO, 2005).

Este texto está organizado da seguinte maneira. No capítulo 1 é feita uma introdução da Tomografia por Impedância Elétrica. No capítulo 3 é feita uma 
revisão bibliográfica sobre os aspectos mais importantes da Tomografia por Impedância Elétrica como também do Método dos Elementos Finitos, modelo de eletrodos, padrões de injeção de corrente e aplicação de Programação Linear a Tomografia por Impedância Elétrica. Em seguida no capítulo 4 é mostrado os diferentes testes que serão realizados para avaliar o desempenho do algoritmo. Nos capítulos 5 e 6 são apresentados os resultados obtidos e uma discussão dos resultados, respetivamente. Finalmente nos capítulos 7 e 8 são mostradas as conclusões e comentarios finais do trabalho. 


\section{Objetivos}

O objetivo principal deste trabalho é investigar se há vantagens em utilizar Programação Linear (PL) como método de busca de imagens na Tomografia por Impedância Elétrica (TIE). As imagens de TIE são classificadas em imagens de variação de resistividade ou em imagens absolutas de resistividade. Correções nas imagens são, em geral, o resultado da solução de um sistema linear do tipo $A x=b$. Os algoritmos atuais, na sua grande maioria, resolvem este sistema algébrico utilizando algoritmos que resolvem o sistema em bloco, por exemplo, LU Decomposition, QR, Gradientes Conjugados, Cholesky Decomposition, para citar alguns. Estes algoritmos bloco requerem matrizes melhor condicionadas do que a Programação Linear, que não requer matrizes bem condicionadas por utilizar restrições que tornam o espaço de solução convexo. Por não requerer matrizes bem condicionadas, a intensidade de regularização necessária para resolver o problema inverso de TIE via PL poderia ser menor. Este trabalho investiga esta proposição, tanto na busca de imagens absolutas quanto na busca de imagens de variação de resistividade. 


\section{Revisão Bibliográfica}

A TIE é uma técnica não invasiva que procura determinar a distribuição de resistividade de um determinado domínio. Com a distribuição de resistividade de uma seção do corpo é possível gerar imagens médicas.

Do ponto de vista da engenharia, a TIE é definida pela maioria dos autores como um problema de estimação de parâmetros de um modelo não linear que representa um domínio do ponto de vista eletromagnético. Supõem-se conhecidos os padrões de corrente que servem de excitação do sistema, a estrutura do modelo, e são medidos os potenciais elétricos no contorno da região.

Para gerar imagens na TIE, têm sido estudados alguns métodos, classificados em métodos iterativos e métodos não-iterativos. Os métodos não-iterativos podem ser resolvidos através de métodos lineares, pois a distribuição de potencial se considera uma função linear da distribuição de condutividade elétrica. No caso dos métodos iterativos, considera-se que existe uma relação não-linear entre os potenciais e a condutividade elétrica e, basicamente, busca-se minimizar um funcional de erro quadrático dado pela diferença entre os potenciais elétricos medidos e os potenciais elétricos calculados. Exemplos de ambos tipos de algoritmos são Backprojection (BARBER; BROWN, 1984) e métodos relacionados (SAntosA; VOGElius, 1990); Newton Raphson (ARUCA, 2002), (DOBSON, 1992), (VANEGAS, 2002); (EGGLESTON et al., 1989); Filtro estendido de Kalman (Trigo, 2001), (VAuHKonen; KARJAlainen; KAIPIO, 1998a); Markov Chain Monte Carlo(MCMC) (KAIPIO et al., 2000).

Em TIE, é comum falar de dois tipos de problemas: Problema Direito e Problema Inverso. No problema direto são definidas as propriedades eletromagnéticas do domínio, correntes de excitação, a estrutura do modelo e o objetivo é calcular os potenciais nos eletrodos. No problema inverso são conhecidos os potenciais nos eletrodos, correntes de excitação, a estrutura do modelo e procura-se conhecer a distribuição das propriedades eletromagnéticas. Para a solução do problema inverso, existe na literatura os métodos absolutos e que geram imagens de vari- 
ações das propriedades eletromagnéticas (VAUHKONEN; KARJALAINEN; KAIPIO, 1998b).

Nos métodos ditos estáticos as propriedades dielétricas do meio em estudo são consideradas constantes ao longo de um intervalo de tempo $\Delta t$, durante o qual ocorre a injeção dos padrões de corrente e a leitura de potencial nos eletrodos. Os métodos ditos dinâmicos, contém na sua formulação modelos de evolução temporal do estado sistema (TRIGO, 2005).

A TIE está sendo aplicada em diagnóstico médico em diferentes áreas tais como funções gástricas e esofágicas, ventilação e edemas pulmonares, derrame cerebral, perfusão torácica, estudo das funções do coração entre outros. Margaret Cheney expõe em Electrical Impedance Tomography (CHENEY; ISAACSON; NEWELL, 1999), as aplicações da TIE em diagnóstico médico e as vantagens que tem quando comparada com outras técnicas entre as quais ressalta que é um método não invasivo e facilmente transportável, apresenta algumas aplicações industriais, mostra o modelo matemático do problema a ser resolvido e algoritmos para encontrar a distribuição de resistividade.

No artigo Three-Dimensional Electrical Impedance Tomography of Human Brain Activity (TIDSWELL et al., 2001) utilizam TIE para monitorar o fluxo e volume de sangue na região cerebral quando acontece atividade no cérebro. A impedância elétrica do cérebro é determinada pelas diferentes impedâncias dos neurônios, sangue e o fluxo extra-celular. O número de eletrodos utilizado para fazer as medições foram 31 e o posicionamento deles seguiu normas internacionais, mas quatro eletrodos foram alocados em posições escolhidas, dois foram posicionados nos ossos atrás das orelhas e os outros dois foram posicionados na parte superior da cabeça.

Em Impedance imaging in the newborn (MURPHY et al., 1987) implementam TIE para monitorar o cérebro dos recém nascidos querendo detectar o mais rápido possível hemorragias intraventriculares as quais são a maior causa de morte em recém nascidos de pouco peso, o esvaziamento gástrico depois de ingerir alimento procurando padrões normais e anormais e o monitoramento dos pulmões dos recém nascidos submetidos a ventilação artificial. O número total de eletrodos usados para injetar corrente e medir potenciais elétricos é 16, alocados equiespaçados ao redor do corpo em estudo.

O Eung Je Woo no artigo Measuring Lung Resistivity Using Electrical Impedance Tomography (WOO et al., 1992) descreve a utilização da TIE para monitorar e detectar a presença de apnéia e edema nos pulmões. O algoritmo de recons- 
trução de imagem é absoluto, aproveitam o conhecimento a priori da estrutura do tórax para agrupar elementos finitos que pertencem ao mesmo órgão e associam a um orgão um único valor de resistividade. O número total de eletrodos implementados é 32 e estão equi-espaçados ao redor do tórax. No artigo Breast Cancer diagnosis and Prognosis Via Linear Programming (MANGASARIAN; STREET; WOLBERG, 1995) a PL, é utilizada no diagnóstico do câncer de mama.

Imagens anatômicas de uma seção transversal dos pulmões podem ser obtidas através de tomografia computadorizada (CT-scan) ou ressonância magnética (MRI). Entretanto, não é possível monitorar um paciente submetido à ventilação artificial com essas técnicas, pois elas emitem radiação ou necessitam de contrastes. A vantagem da TIE reside no fato de ela ser não invasiva, portátil (podendo ser levada à beira do leito) e utilizar o potencial elétrico como estímulo para a obtenção das imagens.

Além disso, a TIE tem um extenso campo de aplicação no setor industrial, algumas das aplicações são: distribuição de sólidos dentro de um hidrociclone (WILLIAMS et al., 1997), imagens de fluxos industriais, alterações de fluxo volumétrico em óleo e gás (FEHMERS, 2003), medidas da distribuição de material em fluxos de duas fases (GEORGE et al., 2000) entre outros.

Em Electrical Impedance Tomography of a Seafloor Volcano (MYER; CONSTABLE; KEY, ) se procura obter imagens do magma vulcânico usando TIE, os eletrodos são enterrados ou colocados dentro de perfurações. O autor R. C. Waterfall em Combustion imaging from electrical impedance measurements (WATERFALL et al., 1996) explica que na queima de combustível nos motores de combustão interna a concentração de íons aumenta o que produz uma variação nas constantes dielétricas do volume de combustão, a aplicação da TIE permite gerar imagens no interior das câmaras de combustão já que o aumento da concentração de íons gera aumento na condutividade dentro da câmara de combustão.

No artigo Performance Assessment of an Electrical Impedance Tomography Detector for Mine-Like Objects (CHURCH et al., ) o objetivo da implementação da TIE é detetar minas terrestres e minas anti-tanques. Na implementação da TIE neste tipo de problema, é preciso prestar atenção ao contato entre o eletrodo e o solo e ao algoritmo de reconstrução de imagem. A tecnologia da TIE é sensível à distância o que limita a implementação da TIE só a objetos enterrados a pouca profundidade.

Na tese de doutorado Estudo da Obtenção de Imagens de tomografia de Impedância Elétrica do Pulmão pelo Método de Otimização Topológica (LIMA, 2006) 
o autor resolve o problema de otimização utilizando o algoritmo conhecido como Programação Linear Seqüencial (PLS). A PLS divide um problema de otimização não-linear em vários problemas de otimização lineares os quais são resolvidos pela PL. Este trabalho sugere que há vantagens numéricas e vantagens quanto a facilidade de regularização do problema quando se utiliza PL.

O método Programação Linear (PL) utilizado neste trabalho (MAKHORIN, 2004) é um método iterativo para busca de distribuições de resistividade que minimizem um índice de performance. Acredita-se possível diminuir os parâmetros de regularização utilizados para melhorar a condição numérica das matrizes através do uso de restrições no algoritmo de PL. As restrições teriam o papel de restringir o espaço solução e introduzir informação a priori no problema inverso de TIE.

\subsection{Formulação Teórica}

\subsubsection{Formulação Teórica do Domínio}

Dado um domínio com propriedades eletromagnéticas desconhecidas, a TIE permite estimar a distribuição de resistividade do domínio, dada a excitação do sistema, composta por correntes impostas no contorno do domínio e conhecidos os potenciais elétricos que também são medidos em regiões do contorno do domínio. Uma particular escolha de amplitude e fase de injeção de corrente em cada eletrodo será chamada de "padrão de corrente ${ }^{1 " .}$

É conhecido que diferentes tecidos do corpo humano tem diferentes propriedades elétricas. As relações que governam as iterações entre a eletricidade e o magnetismo são resumidas nas equações de Maxwell. A maioria dos tecidos, dentro de certo nível de precisão, pode ser considerado isotrópico, a exceção dos músculos e do cérebro que é anisotrópico (ASFAW, 2005). A figura $3.1^{2}$ ilustra diferentes tecidos. Na TIE é possível assumir simplificações que reduzem a complexidade do problema. O corpo humano é assumido isotrópico, então a condutividade independe da direção.

O tórax humano é modelado como um domínio fechado $\Omega$ e limitado por uma superfície de contorno $\partial \Omega$, contendo distribuição de condutividade $\sigma$. Com estas

\footnotetext{
${ }^{1}$ Padrão de corrente é explicado na seção 3.1 .4

${ }^{2}$ Figura extraída do website http://www.medstudents.com.br/caso/caso20/tctorax2.jpg
} 
simplificações as equações de Maxwell são escritas

$$
\nabla \times \mathbf{E}=-\frac{\partial \mathbf{B}}{\partial t} \quad \longrightarrow \quad \oint_{c} \mathbf{E} \cdot d l=-\frac{d}{d t} \int_{s} \mathbf{B} \cdot d \mathbf{A}
$$

onde $\mathbf{E}$ é o campo elétrico e $\mathbf{B}$ é a densidade de fluxo magnético.

No artigo Finite element modeling of electrode-skin contact impedance in Electrical Impedance Tomography (HUA; WEBSTER; TOMPKINS, 1993) mostra que quando a freqüência da corrente que passa pelo meio é inferior a $30 \mathrm{MHz}$ o termo derivada da densidade de fluxo magnético é nulo,

$$
\nabla \times \mathbf{E}=0
$$

Na forma integral a equação 3.2, resulta

$$
\oint_{c} \mathbf{E} \cdot d l=0
$$

Desta última equação, 3.3, pode-se deduzir que existe um potencial escalar $\Psi$ tal que

$$
\mathbf{E}=-\nabla \Psi(\sigma)
$$

Dado que o domínio $\Omega$ não tem fontes de cargas, em qualquer ponto dentro do domínio, o divergente da densidade de corrente $\mathbf{J}$ é nulo:

$$
\nabla \bullet \mathbf{J}=0
$$

A densidade de corrente dentro de um líquido ou sólido é uma função do campo elétrico (STRATton, 1940) $\mathbf{E}$, isto é, J $=\mathbf{J}(\mathbf{E})$. Sendo está função linear e ainda

$$
\mathbf{J}=\sigma \mathbf{E}
$$

Através dos eletrodos $e$ posicionados na fronteira do domínio $\partial \Omega$ são injetadas e medidas correntes e tensões. Então as condições de contorno associadas às correntes podem ser representadas por

$$
\begin{array}{ll}
\sigma \frac{\partial \Psi(\sigma)}{\partial n}=J_{i}, & i=1,2, \ldots, e \\
\sigma \frac{\partial \Psi(\sigma)}{\partial n}=0 & \text { Nos demais pontos da fronteira }
\end{array}
$$

onde $n$ é um versor normal à superfície e orientado para fora e $J_{i}$ é a densidade de corrente no i-ésimo eletrodo.

Neste trabalho, adota-se como variável a ser estimada a resistividade $\rho$ que é 
relacionada com a condutividade por $\rho=\sigma^{-1}$.

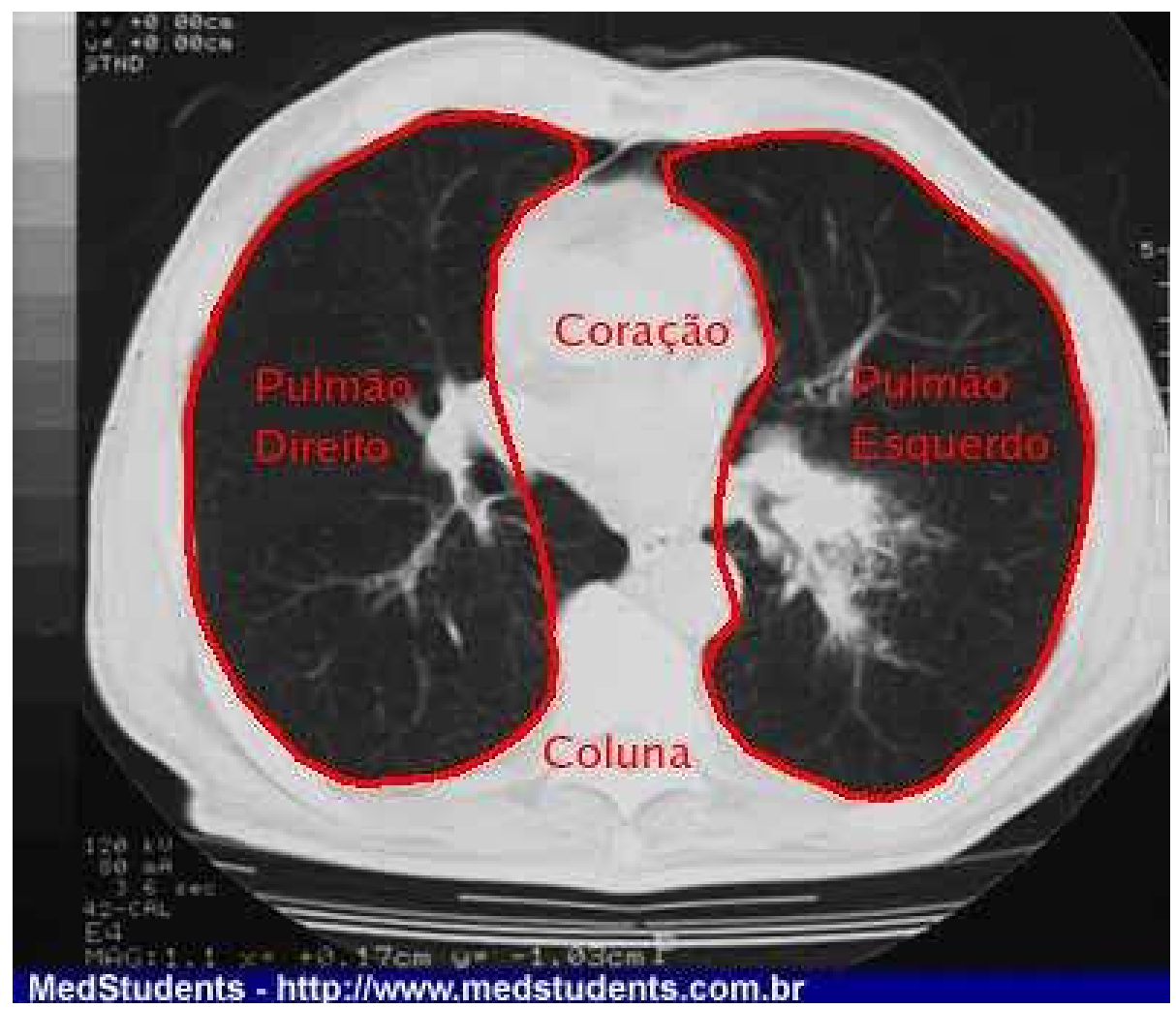

Figura 3.1: Seção Transversal do tórax (imagem modificada para visualizar os órgãos do tórax)

\subsubsection{Método de Elementos Finitos}

No MEF, o domínio em estudo é dividido num número finito de elementos geométricos, em cada um deles o campo contínuo no qual as variáveis da EDP são definidas e modelado por uma aproximação polinomial local controlada por uma pequena quantidade de coeficientes. A conexão desses elementos através dos valores desses coeficientes em posições nodais compartilhadas, resulta em um conjunto de equações algébricas que pode ser resolvido numericamente por meio de métodos de otimização e de algoritmos matriciais ${ }^{3}$.

O domínio de interesse $\Omega$ (Neste trabalho o Tórax) é discretizado em uma malha de $m$ nós e $n$ elementos triangulares com resistividade $\rho_{i}, i=1,2,3 \ldots, n$, como é mostrado na figura 3.2

\footnotetext{
${ }^{3}$ Texto extraído do website http://www.thorus-scisoft.com.br/?q=pt/node $/ 47$
} 


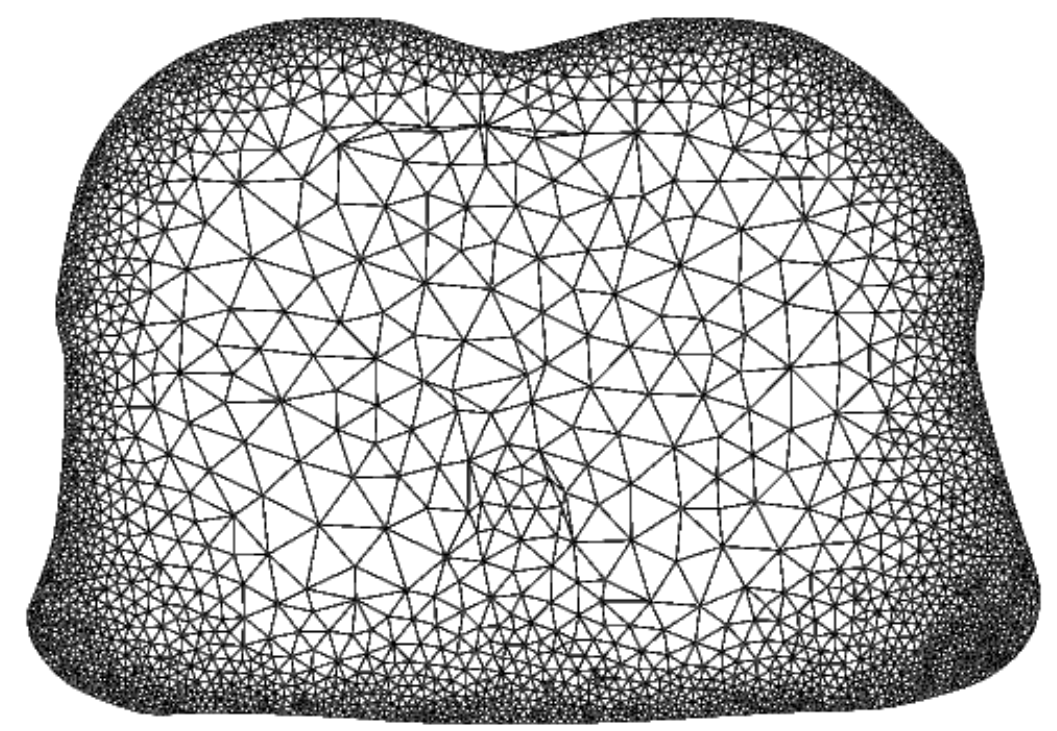

Figura 3.2: Discretização do tórax em elementos triangulares (BORSIC et al., 2000)

Com o domínio já discretizado, procura-se uma aproximação para a distribuição de potencial $v_{e}$ dentro de cada elemento genérico $n$ e então relacionando a distribuição de potencial $v_{e}$ de vários elementos tal que a distribuição de potencial é contínuo através das fronteiras dos elementos relacionados encontra-se uma solução aproximada para a distribuição de potencial no domínio discretizado (SADIKU, 1992)

$$
V(x, y) \simeq \sum_{e=1}^{n} v_{e}(x, y)
$$

Para aproximar $v_{e}$, utiliza-se, por exemplo, a seguinte função de interpolação linear, válida dentro do e-ésimo elemento e nula no resto do domínio $\Omega$,

$$
v_{e}(x, y)=a+b x+c y
$$

onde $a, b$, e $c$ são funções dos potenciais elétricos dos três vértices do elemento triangular.

Foge ao escopo deste trabalho desenvolver as matrizes locais dos elementos triangulares de três nós no método de elementos finitos. Apresentamos aqui apenas a notação que será empregada neste trabalho. Vamos denotar por $v_{e 1}, v_{e 2}$ e $v_{e 3}$ os potenciais elétricos nodais do e-ésimo elemento finito triangular. Vamos denotar por $\mathbf{Y}_{i}^{l} \in \Re^{3 \times 3}$ a matriz de rigidez local do i-ésimo elemento e por $\mathbf{Y} \in$ $\Re^{m \times m}$ a matriz de rigidez global.

O potencial elétrico dentro do e-ésimo elemento finito é função do potencial 
elétrico em cada nó

$$
v_{e}=\sum_{i=1}^{3} \alpha_{i}(x, y) v_{e i}=\left[\begin{array}{lll}
\alpha_{1} & \alpha_{2} & \alpha_{3}
\end{array}\right]\left[\begin{array}{l}
v_{e 1} \\
v_{e 2} \\
v_{e 3}
\end{array}\right]
$$

onde $\alpha_{i}$ são funções de interpolação, equação 3.9 .

Cada elemento triangular tem matriz de rigidez local $\mathbf{Y}_{i}^{l} \in \Re^{3 \times 3}$ que é função da distribuição de resistividade $\rho_{i} \in \Re^{n}$, com as matrizes de rigidez locais calculadas procede-se a montagem da matriz de rigidez global $\mathbf{Y} \in \Re^{m \times m}$. As regras de montagem da matriz global e as expressões da matriz de rigidez local podem ser encontradas em A First Course in the Finite Element Method (LOGAN, 2002), por exemplo.

\subsubsection{Modelo de Eletrodo 2D}

Na TIE, são injetadas correntes e medidos potenciais elétricos através de eletrodos alocados no tórax do paciente. As medidas de potencial elétrico são afetadas pela impedância de contato entre o eletrodo e a pele e pela distribuição de resistividade no domínio $\Omega$.

A queda de potencial elétrico na região de contato eletrodo-pele é significativa, quando o eletrodo é utilizado para injetar corrente, se comparada com a queda de potencial elétrico no interior do domínio. Em humanos, os valores de impedância de contato são da ordem de $800 \Omega \mathrm{cm}^{2}$ (ROSELL et al., 1998), variando com a posição.

O modelo de eletrodo implementado, foi proposto no artigo Finite element modelling of electrode-skin contact impedance in Electrical Impedance Tomography (HUA; WEBSTER; TOMPKINS, 1993). O eletrodo é modelado através do MEF como é mostrado na figura 3.4 .

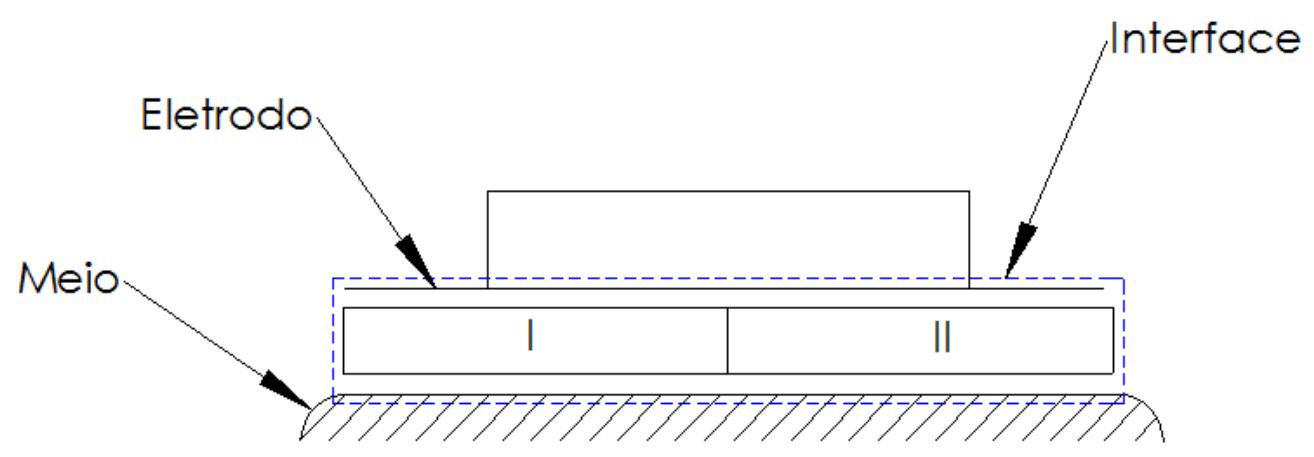

Figura 3.3: Discretização da interface eletrodo-pele 


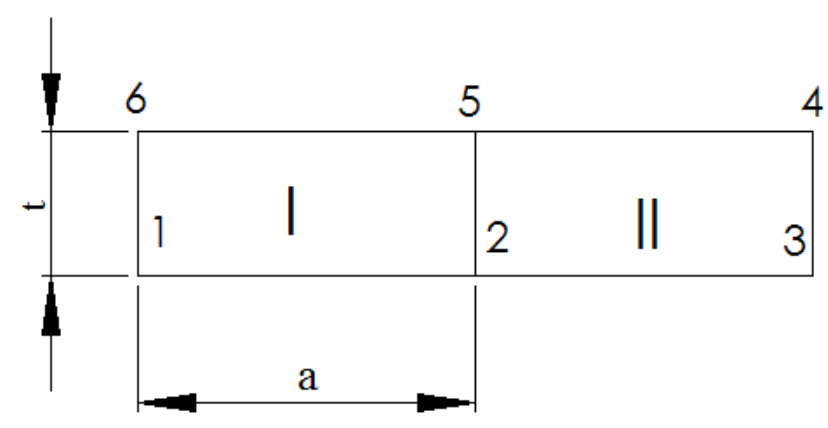

Figura 3.4: Discretização do eletrodo em dois elementos e seis nós

Admite-se que o potencial na parte metálica do eletrodo é uniforme, ou seja o potencial nos nós 4,5 e 6 são iguais, e a espessura da interface do eletrodo $t$ é muito menor que a largura do eletrodo $a(t \ll a)$. Admitidas estás hipóteses chega-se à seguinte expressão matricial para o modelo do eletrodo ${ }^{4}$.

$$
\mathbf{Y}_{i}^{e}=\frac{a b}{3 t \rho}\left[\begin{array}{cccc}
1 & \frac{1}{2} & 0 & -\frac{3}{2} \\
& 2 & \frac{1}{2} & -3 \\
\operatorname{sim} & & 1 & -\frac{3}{2} \\
& & & 6
\end{array}\right]
$$

onde $b$ é a dimensão da interface perpendicular a $a$ e $t, \mathbf{Y}_{i}^{e}$ é a matriz de rigidez do eletrodo. O modelo resultante (3.11) é acrescentado à matriz global do modelo de elementos finitos do tórax para a solução do problema inverso.

\subsubsection{Padrões de Injeção de Corrente e de Medida de Po- tencial}

Na TIE freqüentemente, a corrente é injetada por meio de eletrodos alocados na fronteira do domínio $\Omega$ e os potenciais são medidos nos mesmos eletrodos. Define-se o termo padrão de corrente como a forma em que a corrente é injetada, igualmente define-se o termo padrão de voltagem como a forma em que os potenciais elétricos são medidos.

Quando o circuito eletrônico de medida dos potenciais elétricos tem ganho fixo e nenhum sinal de medida de potencial elétrico satura, os sinais de menor amplitude apresentam baixa relação sinal/ruído devido ao ruído de quantização introduzido na operação de conversão de analógico para digital. Através de uma criteriosa escolha de padrões de injeção de corrente e de padrões de medida de

\footnotetext{
${ }^{4}$ Para ver o desenvolvimento matemático do modelo do eletrodo consultar (HUA; WEBSTER; TOMPKINS, 1993)
} 
potenciais elétricos, é possível minimizar a faixa dinâmica das medidas e aproveitar ao máximo o número de bits do conversor de sinal analógico para sinal digital. Dentre as combinações de padrões de injeção e padrões de medida de potencial elétrico que minimizam a faixa dinâmica das medidas encontram-se os padrões pula um eletrodo e pula três eletrodos descritos nesta seção.

No presente trabalho o método dos padrões pula um eletrodo e pula três eletrodos foram adotados. No método pula um eletrodo, a injeção de corrente é feita em dois eletrodos separados por um eletrodo, por exemplo o par $(1,3)$ conforme mostrado na figura 3.5(a), mantendo a corrente de injeção nos eletrodos $(1,3)$ os potenciais elétricos são medidos entre pares de eletrodos separados também por um eletrodo, desde o par $(1,3)$ até o par $(\mathrm{e}, 2)$, conforme mostrado na figura 3.5(a), sendo e o número total de eletrodos. Feitas estas medições, altera-se o par de injeção em uma unidade e potenciais elétricos são novamente medidos.

O outro método utilizado no presente trabalho para injetar corrente e medir potenciais elétricos, chamado pula três eletrodos difere do método anteriormente descrito somente no número de eletrodos que são pulados, tanto para injetar corrente quanto para medir potencial, que é três, como mostrado na figura $3.5(\mathrm{~b})$.

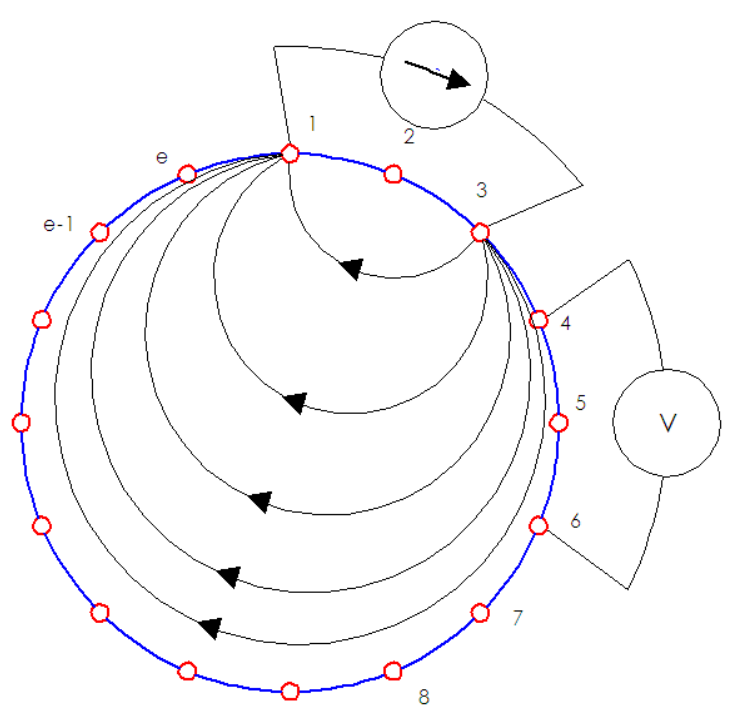

(a)

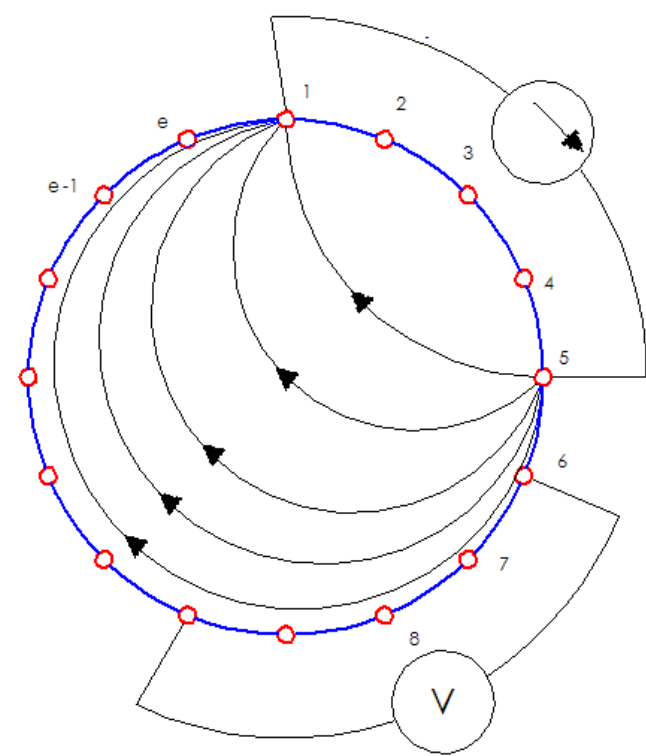

(b)

Figura 3.5: Padrão de corrente (a) pula um eletrodo (b) pula três eletrodos

Comparando os dois métodos, pode-se dizer que no padrão pula um eletrodo a distância entre os eletrodos de injeção é menor, quando comparado com o padrão pula três eletrodos, o que permite obter uma maior densidade de corrente perto da borda, que se reflete numa maior resolução da imagem perto da borda. A 
resolução diminui no centro do domínio, distante do par de injeção. Do ponto de vista da relação sinal/ruído, sendo o ruído nas medições constantes, o padrão pula três eletrodos tem uma melhor relação sinal/ruído já que os sinais de potenciais elétricos são maiores quando comparado com os sinais medidos do padrão pula um eletrodo.

Alguns sistemas não utilizam os três potenciais que envolvem os eletrodos que injetam corrente por estarem influenciados pela impedância de contato meioeletrodos que muitas vezes não é conhecida com precisão. Como os potenciais são medidos entre 2 eletrodos e não entre um eletrodo e uma referência fixa, diz-se que medem-se tensões diferenciais. Este tipo de medida traz a vantagem de reduzir o ruído eletromagnético quando este ocorre simultaneamente nos dois condutores ligados aos eletrodos.

\subsubsection{Solução do Problema Direto}

No Problema Direto são conhecidas as propriedades eletromagnéticas do domínio e as condições de contorno (padrões de corrente injetados nos nós correspondentes aos eletrodos e potencial elétrico de referência) e procura-se determinar os potenciais nos eletrodos. Para determinar os potenciais nos eletrodos é preciso resolver a seguinte equação em função de $\boldsymbol{v}$

$$
\mathrm{Y}(\rho) v=I \Longrightarrow v=\mathrm{Y}(\rho)^{-1} \mathrm{I}
$$

onde $\boldsymbol{v} \in \Re^{m}$ é o vetor dos potenciais nos nós, $\rho \in \Re^{n+e}$ é o vetor de resistividade dos elementos e dos eletrodos e $\boldsymbol{I} \in \Re^{m}$ é o vetor da corrente injetada. Dado que a matriz $\mathbf{Y}$ é singular, escolhe-se um nó arbitrário $q$ como terra, para torná-la não singular e inversível. A linha $j$-ésima e a coluna $j$-ésima da matriz $\mathbf{Y}$ são igualadas a zero e a correspondente posição $j$-ésima do vetor $\boldsymbol{I}$, a intersecção entre a linha $j$-ésima e a coluna $j$-ésima da matriz $\mathbf{Y}$ é igualada a 1 (YORKEY; WEBSTER; TOMPKINS, 1987). Feito este procedimento a singularidade da matriz $\mathbf{Y}$ é eliminada e o vetor $\boldsymbol{v}$ é determinado. A singularidade da matriz $\mathbf{Y}$ reflete a arbitrariedade da referência de potencial elétrico.

\subsubsection{Solução do Problema Inverso}

O problema inverso busca o vetor de distribuição de resistividade ${ }^{5}, \rho \in \Re^{n+e}$ partindo dos dados de potencial medidos, das correntes elétricas injetadas e da

\footnotetext{
${ }^{5} \mathrm{~A}$ resistividade encontrada é só para os $n$ elementos, já que a resistividade dos $e$ eletrodos assume-se conhecida.
} 
estrutura do modelo do domínio. Como a distribuição de resistividade é desconhecida, então, a matriz de rigidez local $\mathbf{Y}_{i}^{l}$ de cada elemento triangular assim como matriz de rigidez global $\mathbf{Y}$ são escritas em função da resistividade.

Inicialmente adotam-se valores de resistividade de cada elemento. Calculamse potenciais através do problema direto. Conhecidos potenciais calculados e potenciais medidos, calcula-se uma primeira correção do vetor de resistividade seguindo equações que decorrem analíticamente da busca de um extremo de I.E.

$$
\frac{\partial I \cdot E .}{\partial \rho}=0
$$

onde I.E é uma medida da distância entre os potenciais medidos e os potenciais calculados. Corrige-se o vetor de resistividade e repete-se o procedimento anterior para gerar uma nova correção no vetor de resistividade.

\subsubsection{Restrição de Inclinação Máxima na Distribuição de Resistividade (RIM)}

A restrição de inclinação máxima na distribuição de resistividade (RIM), visa introduzir informação a priori diminuindo as alterações de resistividade em regiões do domínio onde a distribuição de resistividade é suave. O objetivo da RIM é penalizar a diferença de resistividades de elementos que estão dentro de um rádio de cobertura $\boldsymbol{R}$ a um valor de inclinação máxima $\mu$ mantendo a diferença de resistividade entre elementos vizinhos restringida ao valor da inclinação $\mu$. Para tentar diminuir o efeito de coroa presente nas imagens causado pela modelagem pouco refinada da região do eletrodo, os elementos que estão perto da borda vão ser penalizados com um valor de inclinação menor.

Partindo da figura 3.6, para cada elemento da malha, determinam-se quais elementos estão dentro de uma circunferência de radio $\mathbf{R}$. Para determinar se os elementos vizinhos ao elemento escolhido estão dentro da circunferência, calculase a distância entre centroides e compara-se com $\mathbf{R}$. 


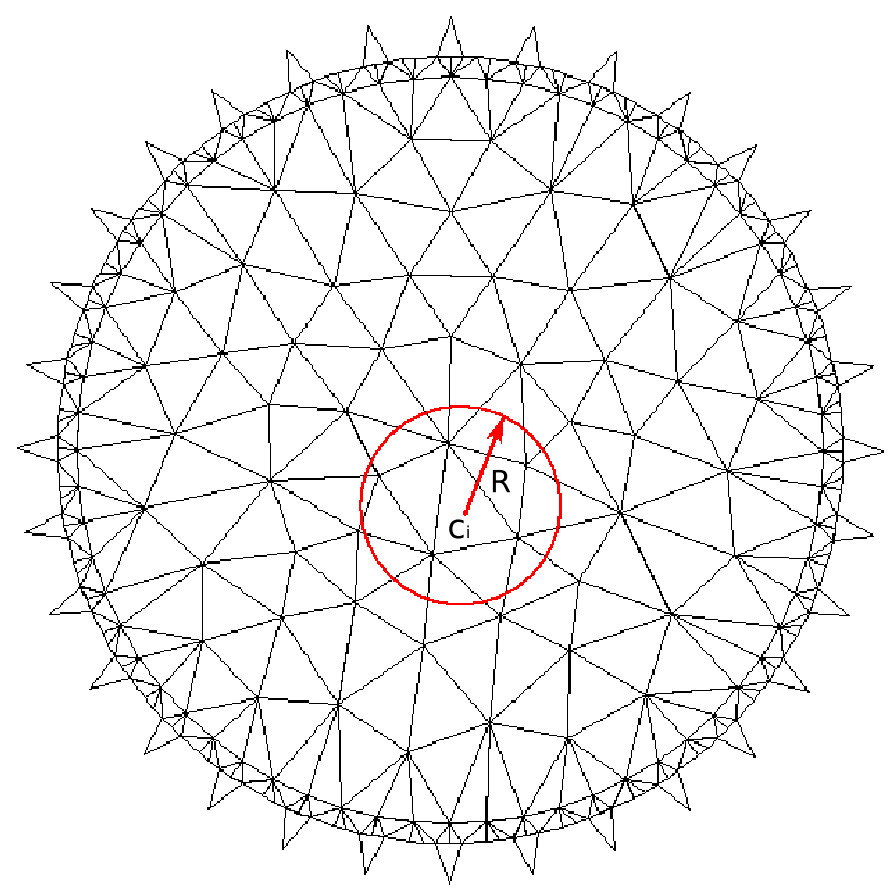

Figura 3.6: Determinação da circunferência de radio $\mathbf{R}$ dentro da qual buscam-se os vizinhos do elemento escolhido

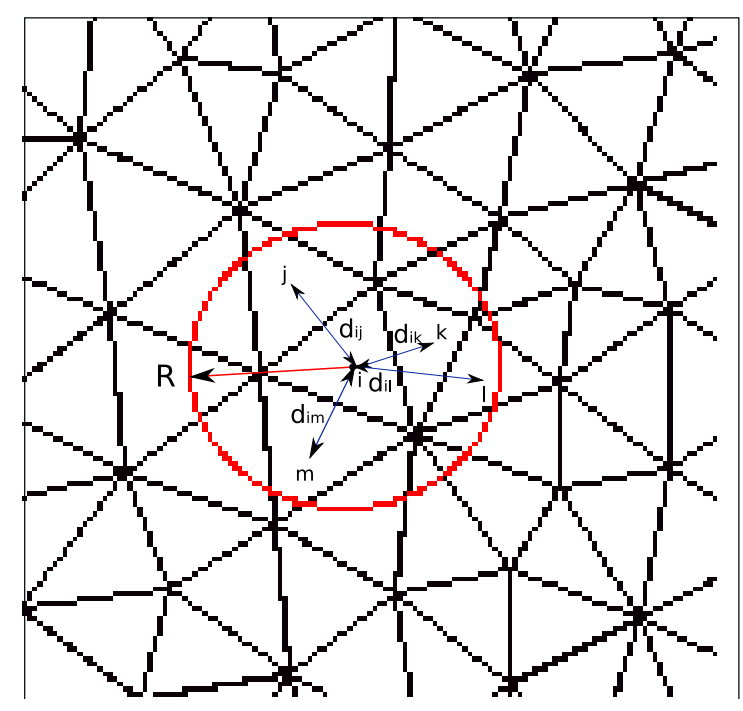

Figura 3.7: Procurando os vizinhos do elemento $i$ e determinando as distâncias aos elementos vizinhos

Escolhido o elemento $i$, como mostrado na figura 3.7, seus elementos vizinhos estão dentro da circunferência de radio $\mathbf{R}$, são os elementos $j, k, l$ e $m$. Determinam-se as distâncias entre os centroides do elemento $i$ com seus vizinhos $\left(d_{i j}, d_{i k}, d_{i l}, d_{i m}\right)$. A diferença de resistividade entre o elemento $i$ e cada um de seus vizinhos podem ser restritas a um valor menor o igual a $\mu$ vezes a distância entre centróides 


$$
\begin{aligned}
& \left|\rho_{i}-\rho_{j}\right| \leq \mu * d_{i j} \\
& \left|\rho_{i}-\rho_{k}\right| \leq \mu * d_{i k} \\
& \left|\rho_{i}-\rho_{l}\right| \leq \mu * d_{i l} \\
& \left|\rho_{i}-\rho_{m}\right| \leq \mu * d_{i m}
\end{aligned}
$$

Representando as equações de 3.14 em forma matricial

$$
\underbrace{\left[\begin{array}{ccccc}
1 & -1 & 0 & 0 & 0 \\
1 & 0 & -1 & 0 & 0 \\
1 & 0 & 0 & -1 & 0 \\
1 & 0 & 0 & 0 & -1
\end{array}\right]}_{\mathbf{S}} \underbrace{\left[\begin{array}{c}
\rho_{i} \\
\rho_{j} \\
\rho_{k} \\
\rho_{l} \\
\rho_{m}
\end{array}\right]}_{\rho} \leq \underbrace{\left[\begin{array}{l}
\mu * d_{i j} \\
\mu * d_{i j} \\
\mu * d_{i k} \\
\mu * d_{i l} \\
\mu * d_{i m}
\end{array}\right]}_{\mathbf{c}}
$$

$$
[\mathbf{S}]\{\rho\} \leq\{\mathbf{c}\}
$$

\subsection{Programação Linear}

\subsubsection{Definição de Programação Linear}

A Programação Linear (PL) surgiu como uma técnica de planejamento de atividades, costuma-se dizer também, que a PL é um tópico da ciência pesquisa operacional, a qual contém outros tópicos tais como teoria das filas, simulação, teoria dos Jogos, programação dinâmica, etc (PRADO, 1999).

Pode-se conceituar PL como é mostrado na seguinte figura 


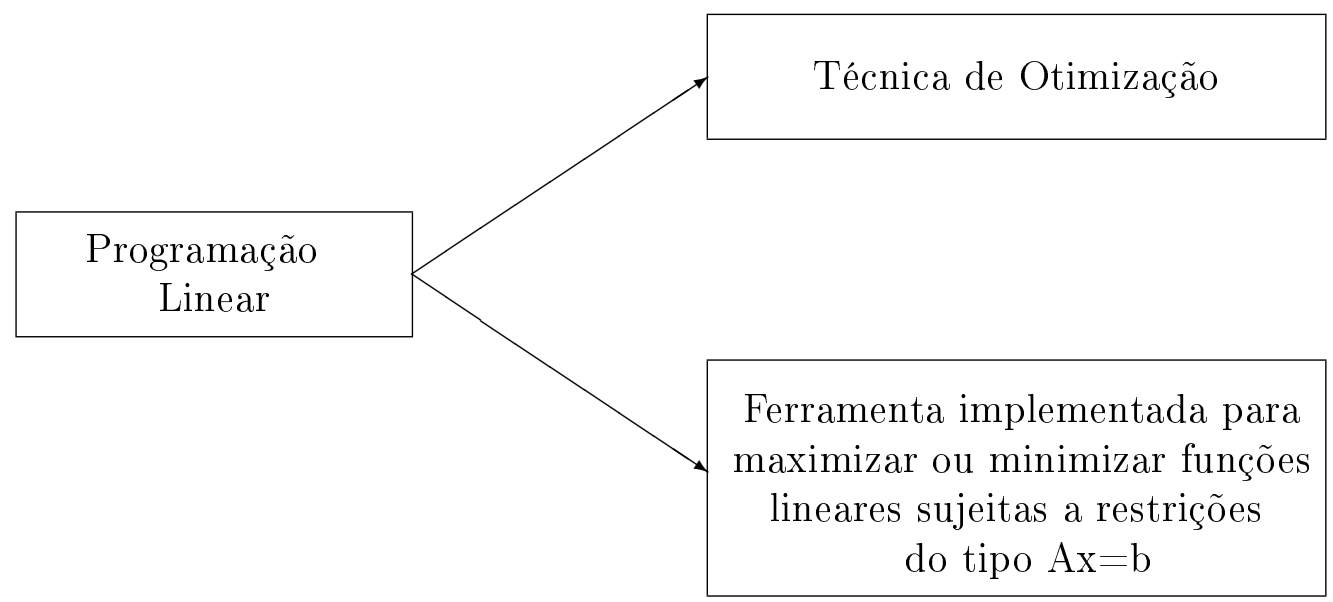

Figura 3.8: Conceituação de PL

\subsubsection{Aplicações Industriais}

As primeiras aplicações de PL foi no campo militar, entre os anos 1955 - 1960 as aplicações de PL cresceram consideravelmente nos campos industrial e comercial.

Na indústria, a aplicação de PL permite o seguimento das operações, análise numérico da estrutura do sistema industrial e o aumento da eficiência nas operações. Além disso, com a aplicação de PL tem sido possível pesquisar e desenvolver modelos quantitativos de sistemas industriais com o objetivo de fornecer cronogramas ótimos para a produção.

A maior implementação de PL é na indústria do petróleo (CHARNES; COOPER; MELLON, 1952), onde é utilizada para gerar cronogramas de produção, também é implementada em exploração, distribuição e refinamento do petróleo. A rotina de carregar os navios cisterna é feita com ajuda de PL.

O segundo campo de aplicação é na indústria de processamento de alimentos. No ano 1953, um produtor industrial implementou PL para determinar a distribuição de ketchup por barco, desde 6 plantas de produção até 70 pontos de venda (HENDERSON; SCHLAIFER, 1954). Na indústria do ferro e aço, PL foi usada para avaliar os diferentes minerais de ferro e a peletização de minerais de baixo grau (FABIAN; TIBOR, 1954).

\subsubsection{Formulação Matemática}

A PL consiste na determinação de valores para as variáveis $x_{1}, x_{2}, \ldots, x_{n}$ a satisfazer um sistema de $m$ equações ou inequações lineares e que maximizem 
(minimizem) uma função (real) linear dessas variáveis.

Algebricamente tem-se :

$$
\begin{aligned}
& \operatorname{Max} \text { ou Min } Z(x)=c_{1} x_{1}+c_{2} x_{2}+\cdots \cdots+c_{n} x_{n} \\
& x
\end{aligned}
$$

Sujeito a

$$
\begin{aligned}
& a_{11} x_{1}+a_{12} x_{2}+\cdots+a_{1 j} x_{j}+\cdots+a_{1 n} x_{n}(\leq,=, \geq) b_{1} \\
& a_{21} x_{1}+a_{22} x_{2}+\cdots+a_{2 j} x_{j}+\cdots+a_{2 n} x_{n}(\leq,=, \geq) b_{2} \\
& \begin{array}{ccccc}
\vdots & \vdots & \vdots & \vdots & \vdots
\end{array} \\
& a_{i 1} x_{1}+a_{i 2} x_{2}+\cdots+a_{i j} x_{j}+\cdots+a_{i n} x_{n}(\leq,=, \geq) \quad b_{i} \\
& a_{m 1} x_{1}+a_{m 2} x_{2}+\cdots+a_{m j} x_{j}+\cdots+a_{m n} x_{n}(\leq,=, \geq) b_{m}
\end{aligned}
$$

onde $a_{i j}, b_{i}$ e $c_{j}$ com $i=1,2, \ldots \ldots, m$ e $j=1,2, \ldots \ldots, n$ são constantes e em cada restrição apenas se verifica uma e uma só das relações,$\leq,=$ ou $\geq$.

A função a maximizar ou minimizar, $Z(x)=c_{1} x_{1}+c_{2} x_{2}+\cdots \cdots+c_{n} x_{n}$, designa-se função objetivo. As equações e inequações designam-se por restrições. As variáveis $x_{j}$ são chamadas variáveis principais, de decisão ou controláveis e constituem as variáveis do modelo; as constantes $a_{i j}, b_{i}$ e $c_{j}$ são chamados coeficientes técnicos.

\subsubsection{Como Utilizar PL na TIE}

Uma maneira de utilizar PL em TIE é na solução de sistemas lineares do tipo $\mathbf{A x}=\mathbf{b}$ aproveitando a grande vantagem de poder colocar restrições nas variáveis $\mathbf{x}$.

O problema de identificar as distribuições de resistividade dentro do tórax tem restrições naturais, a resistividade não pode ser negativa nem pode ultrapassar um valor máximo característico de tecido biológicos. Estas restrições tornam o espaço solução convexo, o que simplifica a solução do problema inverso.

Para conseguir resolver o sistema linear é necessário re-escrever o problema apresentado na equação (3.17). Escolhe-se um vetor arbitrário $\mathbf{x}_{0} \in \Re^{n}$ e procedese a determinar o vetor $\mathbf{e} \in \Re^{n} \operatorname{com} \mathbf{e}=\mathbf{A} \mathbf{x}_{0}-\mathbf{b}$. 
O sistema de restrições original na forma $\mathbf{A x}=\mathbf{b}$ é agora mudado como apresentado na seguinte equação

$$
\mathbf{A x}-\mathbf{b}+\xi \mathbf{e}=0
$$

O problema da equação 3.18 é reformulado para PL

$$
\begin{gathered}
\text { Minimizar } \\
(\mathbf{x}, \xi)
\end{gathered}
$$

Sujeito a

$$
\begin{gathered}
\mathbf{A} \mathbf{x}-\mathbf{b}+\xi \mathbf{e}=0 \\
x_{\min } \leq \mathbf{x} \leq x_{\max } \\
0 \leq \xi \leq \epsilon
\end{gathered}
$$

Quando $\xi \approx 0$, obtém-se que o vetor solução $\mathbf{x}$ satisfaz $\mathbf{A x}=\mathbf{b}$.

\subsection{Programação Linear Seqüencial (PLS)}

Na Programação Linear Seqüencial (PLS), a solução de um problema não-linear é feita pela aproximação de sucessivos problemas lineares os quais são resolvidos usando PL . Cada subproblema de PL é gerado aproximando a função objetivo e as restrições não-lineares usando uma série de Taylor truncada e limitando a variação do valor de cada variável de projeto $\mathbf{X}_{\mathbf{i}}$ em cada subproblema linear através de limites móveis. O problema de PL é resolvido utilizando o método Simplex o qual vai gerar o novo vetor de variáveis de projeto $\mathbf{X}_{\mathbf{i}+\mathbf{1}}$. Se o novo vetor $\mathbf{X}_{\mathbf{i}+\mathbf{1}}$ não satisfaz os critérios de convergência, o problema é relinearizado novamente no ponto $\mathbf{X}_{\mathbf{i}+\mathbf{1}}$ e o procedimento continua até encontrar a solução ótima $\mathbf{X}^{*}$.

Para entendermos melhor o funcionamento do método, são apresentados a seguir as diferentes etapas do método:

1. Escolha de um ponto inicial $\mathbf{X}_{\mathbf{i}}$.

2. Linearizar a função objetivo e as restrições no ponto $\mathbf{X}_{\mathbf{i}}$ como :

$$
\begin{aligned}
& f(\mathbf{X}) \approx f\left(\mathbf{X}_{\mathbf{i}}\right)+\nabla f\left(\mathbf{X}_{\mathbf{i}}\right)^{T}\left(\mathbf{X}-\mathbf{X}_{\mathbf{i}}\right) \\
& g_{j}(\mathbf{X}) \approx g_{j}\left(\mathbf{X}_{\mathbf{i}}\right)+\nabla g_{j}\left(\mathbf{X}_{\mathbf{i}}\right)^{T}\left(\mathbf{X}-\mathbf{X}_{\mathbf{i}}\right) \\
& h_{k}(\mathbf{X}) \approx h_{k}\left(\mathbf{X}_{\mathbf{i}}\right)+\nabla h_{k}\left(\mathbf{X}_{\mathbf{i}}\right)^{T}\left(\mathbf{X}-\mathbf{X}_{\mathbf{i}}\right)
\end{aligned}
$$


3. Formular o novo problema de PL como

$$
\begin{aligned}
& \text { Minimizar } f\left(\mathbf{X}_{\mathbf{i}}\right)+\nabla f\left(\mathbf{X}_{\mathbf{i}}\right)^{T}\left(\mathbf{X}-\mathbf{X}_{\mathbf{i}}\right) \\
& \text { Sujeito a } \\
& g_{j}\left(\mathbf{X}_{\mathbf{i}}\right)+\nabla g_{j}\left(\mathbf{X}_{\mathbf{i}}\right)^{T}\left(\mathbf{X}-\mathbf{X}_{\mathbf{i}}\right) \leq 0, \quad j=1,2, \ldots, m \\
& h_{k}\left(\mathbf{X}_{\mathbf{i}}\right)+\nabla h_{k}\left(\mathbf{X}_{\mathbf{i}}\right)^{T}\left(\mathbf{X}-\mathbf{X}_{\mathbf{i}}\right)=0, \quad i=1,2, \ldots, p
\end{aligned}
$$

4. Resolver o problema de PL para obter o novo vetor $\mathbf{X}_{\mathbf{i}+\mathbf{1}}$

5. Avaliar as restrições do problema não-linear no novo ponto $\mathbf{X}_{\mathbf{i}+\mathbf{1}}$. Se todas as restrições são satisfeitas o ponto $\mathbf{X}_{\mathbf{i}+\mathbf{1}}$ é considerado ótimo $\mathbf{X}_{\mathbf{o p t}}$. Caso contrário, a restrição que não é satisfeita é linearizada no ponto $\mathbf{X}_{\mathbf{i}+\mathbf{1}}$

6. Voltar para o item 2.

Na literatura encontram-se diferentes trabalhos onde é aplicado PLS, entre os quais, destaca-se a implementação feita por (LIMA, 2006) e (MELLO, 2005) onde a PLS resolve um problema não-linear cuja função objetivo é o quadrado da diferença entre os potenciais elétricos calculados e os potenciais elétricos medidos nos eletrodos sujeito a restrições lineares. A solução encontrada por PLS está relacionada com o problema da determinação dos limites móveis o qual será tratado na próxima seção.

\subsubsection{Limites Móveis}

Antes de descrever o procedimento de determinação dos limites móveis definem-se os limites inferior e superior absolutos. O limite superior absoluto $\rho^{\text {sup }}$ e o limite inferior absoluto $\rho^{\text {inf }}$ são definidos tendo em conta o particular problema biológico ou físico. Fazem parte de um conjunto de informações que são conhecidas a priori.

Nos testes planejados na metodologia e detalhados na seção 4.1, o limite inferior absoluto de resistividade $\rho^{\text {inf }}$ adotado é nulo. Esta restrição é chamada na literatura por não-negatividade da resistividade. O limite de resistividade superior absoluto normalmente depende da natureza do domínio, por exemplo, no tórax, durante a ocorrência de um pneumotorax, o limite superior absoluto $\rho^{\text {sup }}$ de resistividade poderia ser de $10^{6} \Omega m$. ' Os limites móveis são os valores máximos e mínimos em que a aproximação por $f(\mathbf{x})$ é razoável. O grande problema de utilizar PLS é a determinação dos limites móveis, uma escolha equivocada dos limites móveis pode acarretar na obtenção de uma imagem errônea ou num custo computacional extremamente elevado. 
Diferentes trabalhos apresentam diferentes métodos de calcular os limites móveis corretamente, (LIMA, 2006) cálcula os limites móveis usando um método heurístico proposto por (BELEGUNDU; ARORA, 1985) que tem em conta a variação do sinal da variável do problema. O autor (CARDOSO, 2000) utiliza uma variação do método utilizado por (LELLEP; MAJAK, 1999) onde a variação de cada variável de projeto é analisada. O trabalho apresentado por (POURAZADY; FU, 1996) calcula os limites móveis tendo em conta o número de iterações do algoritmo, escolhe um valor $\tau$ inicial de partida e dependendo se o valor inicial é maior ou menor que um a redução dos limites móveis é proporcional $\tau^{\frac{1}{n}}$ ou $\tau^{n}$ respectivamente, sendo $n$ o número da iteração.

Os limites móveis devem sempre respeitar o limite superior absoluto e o limite

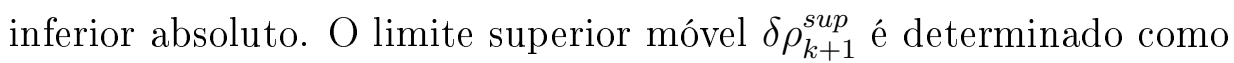

$$
\delta \rho_{k+1}^{\text {sup }}=\beta * \rho_{k+1}
$$

onde $\beta$ é um número real que controla o tamanho do passo durante uma iteração de PLS e $\rho_{k+1}$ é o vetor distribuição de resistividade ainda não calculado. O vetor $\rho_{k}$ foi calculado na iteração anterior.

O limite inferior móvel $\delta \rho_{k+1}^{i n f}$ é determinado por

$$
\delta \rho_{k+1}^{i n f}=-\beta * \rho_{k+1}
$$

onde $\beta$ é um número real que controla o tamanho do passo durante uma iteração de PLS e $\rho_{k+1}$ é o vetor distribuição de resistividade ainda não calculado. O vetor $\rho_{k}$ foi calculado na iteração anterior. A figura 3.9 justifica geometricamente a equação 3.23 e a equação 3.24 .

Lembrando que na iteração $k+1$ do método Gauss-Newton o novo vetor de resistividade $\rho_{k+1}$ será $\rho_{k+1}=\rho_{k}+\delta \rho_{k}$ pode-se escrever os limites móveis como

$$
\begin{array}{r}
\delta \rho_{k+1}^{\text {sup }}=\beta *\left(\rho_{k}+\delta \rho_{k}\right) \\
\delta \rho_{k+1}^{\text {inf }}=-\beta *\left(\rho_{k}+\delta \rho_{k}\right)
\end{array}
$$

sendo $\rho_{k}$ o vetor de resistividade encontrado na iteração $k$ e $\delta \rho_{k}$ é a correção na resistividade calculada usando restrições em função de $\delta \rho_{k+1}^{\text {sup }}$ e $\delta \rho_{k+1}^{\text {inf }}$. O valor de $\beta$ escolhe-se grande (entre $20 \%$ até $40 \%$ ) para a primeira iteração do Gauss- 


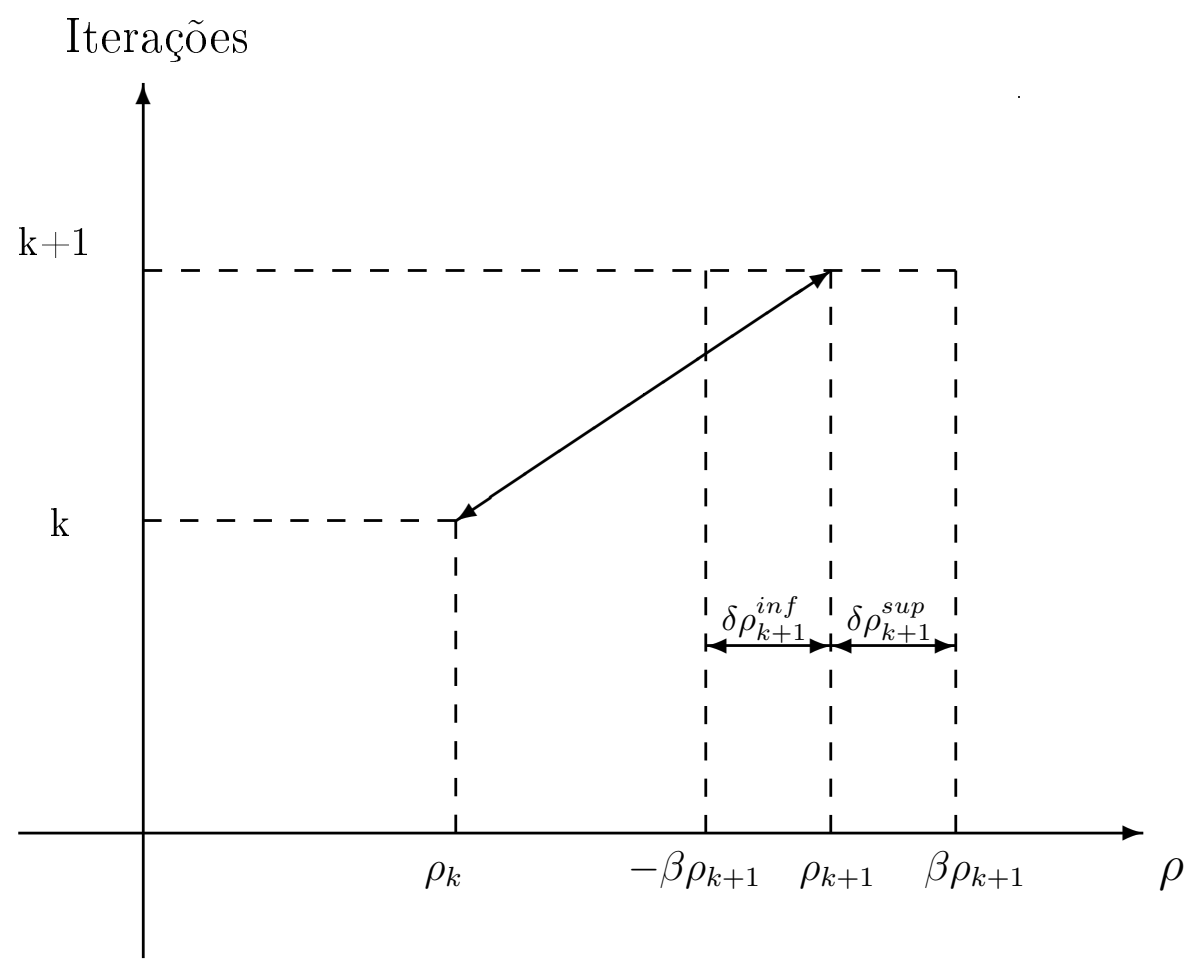

Figura 3.9: Determinação dos limites móveis na iteração $k+1$ do método Gauss-Newton

Newton. A partir da segunda iteração até a p-ésima iteração, que é a última iteração, $\beta$ vai sendo diminuido tendo em conta o comportamento da diferença entre o potencial medido e o potencial calculado, conforme a tabela 3.1, onde o vetor $V_{c}$ é o vetor de potenciais elétricos calculados e $V_{m}$ é o vetor de potenciais elétricos medidos.

\begin{tabular}{|c|c|}
\hline condição & decisão \\
\hline$\left\|V_{m}^{k+1}-V_{c}^{k+1}\right\| \leq\left\|V_{m}^{k}-V_{c}^{k}\right\|$ & $\beta$ não é diminuído \\
$\left\|V_{m}^{k+1}-V_{c}^{k+1}\right\| \geq\left\|V_{m}^{k}-V_{c}^{k}\right\|$ & $\beta$ é diminuído um $10 \%$ \\
\hline
\end{tabular}

Tabela 3.1: Diminuição dos limites móveis

\subsection{O Algoritmo Simplex}

O Simplex pode ser entendido como um mecanismo cuja finalidade é gerar soluções básicas viáveis ${ }^{6}$. O conjunto de soluções viáveis do problema de PL é um conjunto convexo, admitindo que o problema tenha uma solução ótima esta será atingida para, ao menos, um vértice do conjunto de soluções viáveis.

\footnotetext{
${ }^{6}$ Entende-se por solução viável uma solução na qual todas as restrições são satisfeitas (as restrições propriamente ditas e as de não negatividade)
} 


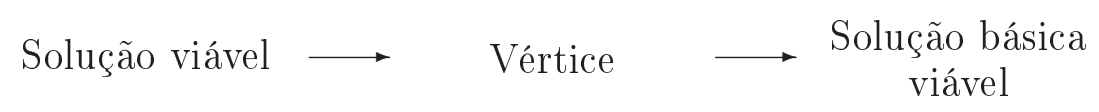

Partindo de uma solução básica viável inicial, o Simplex nada mais é do que um algoritmo capaz de gerar novas soluções básicas viáveis cada vez melhores, até chegarmos a uma que não pode mais ser melhorada. Trata-se neste caso da solução ótima do problema de PL.

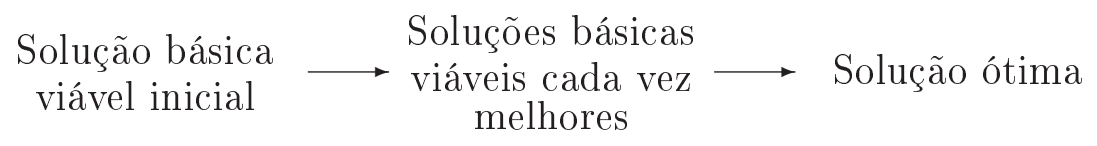

A seguir serão apresentados os diferentes passos do algoritmo simplex, partindo do problema apresentado na equação (3.17) mostrado na forma matricial

$$
\text { Max ou Min } Z(x)=\sum_{j=1}^{n} c_{j} x_{j}
$$

Sujeito a

$$
\begin{gathered}
\sum_{j=1}^{n} a_{i j} x_{j} \leq b_{i} \quad i=1,2, \ldots, m \\
x_{j} \geq 0
\end{gathered}
$$

Adicionando as variáveis artificiais, $x_{n+1}$ até $x_{n+m}$, para reduzir o problema à forma padrão

$$
\begin{aligned}
& a_{11} x_{1}+\cdots+a_{1 j} x_{j}+\cdots+a_{1 n} x_{n}+x_{n+1}=b_{1} \\
& a_{r 1} x_{1}+\cdots+a_{r j} x_{j}+\cdots+a_{r n} x_{n}+\cdots+x_{n+r}=b_{r} \\
& a_{m 1} x_{1}+\cdots+a_{m j} x_{j}+\cdots+a_{m n} x_{n}+\cdots \cdots+x_{n+m} \quad=b_{m} \\
& c_{1} x_{1}+\cdots+c_{j} x_{j}+\cdots+c_{n} x_{n} \quad=Z(x) \\
& x_{j} \geq 0 \quad(j=1,2, \cdots, n+m)
\end{aligned}
$$

Define-se o termo solução básica como qualquer conjunto de $\mathrm{n}$ valores nulos, para $\mathrm{n}$ das variáveis, e m valores não nulos, para as restantes variáveis, que resultem da resolução do sistema de m equações lineares apresentado acima. Podemos representar as $m$ primeiras equações e a função objetivo através do seguinte quadro ou tableau 


\begin{tabular}{|c|c|c|c|c|c|c|c|c|c|}
\hline$x_{1}$ & . & $x_{s}$ & $x_{n}$ & $x_{n+1}$ & $\ldots$ & $x_{n+r}$ & $\ldots$ & $x_{n+m}$ & $b$ \\
\hline$a_{11}$ & $\ldots$ & $a_{1 s}$ & $\ldots a_{1 n}$ & 1 & . & 0 & & 0 & $b_{1}$ \\
\hline$\vdots$ & & $\vdots$ & & $\vdots$ & & $\vdots$ & & $\vdots$ & $\vdots$ \\
\hline$a_{r 1}$ & $\ldots$ & $a_{r s}$ & $\ldots a_{r n}$ & 0 & $\ldots$ & 1 & $\ldots$ & 0 & $b_{r}$ \\
\hline$\vdots$ & & $\vdots$ & & $\vdots$ & & $\vdots$ & & $\vdots$ & $\vdots$ \\
\hline$a_{m 1}$ & $\ldots$ & $a_{m s}$ & $\ldots a_{m n}$ & 0 & $\ldots$ & 0 & $\ldots$ & 1 & $b_{m}$ \\
\hline$c_{1}$ & & $c_{s}$ & . . $\quad c_{n}$ & ( & & 0 & & 0 & $Z(x)$ \\
\hline
\end{tabular}

Tabela 3.2: Representação das primeiras m equações e da função objetivo

Os passos do algoritmo simplex são descritos a seguir.

\subsubsection{Passo 1. Solução Básica Viável Inicial $x$}

Seja o problema (3.27) reduzido à forma padrão e representado pela tabela 3.4. Uma solução básica viável inicial seriam as primeiras $n$ variáveis, iguais a zero e cada variável auxiliar igual a um dos elementos do vetor $\boldsymbol{b}$.

$$
\begin{aligned}
& \mathrm{VNB} \longrightarrow x_{1}=x_{2}=\ldots=x_{s}=\ldots=x_{n}=0 \\
& \mathrm{VB} \longrightarrow x_{n+1}=b_{1}, \ldots, x_{n+m}=b_{m}
\end{aligned}
$$

\subsubsection{Passo 2. É Possível uma Solução Melhor ? Em Caso Afirmativo, Escolher Nova Variável Básica $\boldsymbol{x}$}

O seguinte passo será gerar uma nova solução básica viável melhor que $x$ o que implica necessariamente em ao menos uma nova variável básica, bem como na transformação de uma variável básica em não básica. Atribuindo um valor positivo a uma das variáveis $x_{1}, \ldots, x_{n}$, de forma a anular uma das variáveis $x_{n+1}$, $\ldots, x_{n+m}$. Para a escolha da nova variável básica devemos escolher $x_{s}$ tal que $c_{s}<0$. Neste caso, a função objetivo diminuirá da quantidade $c_{s} x_{s}$. Caso exista mais de um coeficiente negativo da função objetivo, escolhe-se $x_{s}$ tal que

$$
\begin{array}{r}
c_{s}=\min \left(c_{i}\right) \\
i \mid c_{i}<0
\end{array}
$$

Não existindo coeficiente negativo, $c_{i} \geq 0$ torna-se impossível diminuir o valor da função objetivo. Logo, $Z(x)$ representa o mínimo, ou seja $x$ é solução ótima do problema de PL. Caso $c_{i} \leq 0 \forall i$, existe ao menos uma variável não básica tal que 
$c_{s}=0$, fazendo $x_{s}$ variável básica, geramos uma nova solução básica viável $x$.

\subsubsection{Passo 3. Atribuição de Valor à Nova Variável Básica; Determinação da Nova Variável não Básica}

Uma vez escolhida, no passo 2, a nova variável básica, atribui-se o valor $x_{s}=$ $b_{r} / a_{r s}=\min \left(b_{i} / a_{i s}\right)$. O escalar $a_{r s}$ recebe a denominação de pivô, a r-ésima linha é a linha pivô, enquanto a s-ésima coluna é denominada coluna pivô.

Para passar de uma solução básica viável inicial $x$ a uma nova solução básica viável $x$, é importante que o problema de PL estivesse na forma canônica, o que, na verdade, significa a aplicação do método de Gauss-Jordan. Para gerar uma nova solução melhor partindo de $x$ é preciso manter a forma canônica, isto se dá através das operações de pivotamento, discriminadas a seguir com referência ao quadro (3.4.3)

- Dividir a linha pivô pelo pivô $a_{r s}$.

- Anular todos os demais elementos da coluna pivô. Isto poderá ser feito subtraindo da i-ésima linha $(i=1,2, \ldots, r-1, r+1, \ldots, m, m+1)$ a nova linha pivô, multiplicada, respectivamente, por $a_{1 s}, a_{2 s}, \ldots, a_{r-1, s}, a_{r+1, s}, \ldots, a_{m s}, c_{s}$.

Em síntese, o que fazemos nas operações de pivotamento é reduzir o vetor $a_{s}$, que entra na base, a um vetor unitário, substituindo assim o vetor $a_{n+r}$ que sai da base. Realizadas as operações de pivotamento, o quadro (3.4.3) fica assim

\begin{tabular}{|c|c|c|c|c|c|c|c|c|c|}
\hline$x_{1}$ & & $x_{s}$ & $x_{n}$ & $x_{n+1}$ & $\ldots$ & $x_{n+r}$ & $x_{n+m}$ & & $b$ \\
\hline$a_{11}-\frac{a_{r 1} a_{1 s}}{a_{r s}}$ & $\ldots$ & 0 & $a_{1 n}-\frac{a_{r n} a_{1 s}}{a_{r s}}$ & & 1 & $\ldots$ & $-\frac{a_{1 s}}{a_{r s}}$ & 0 & $b_{1}-\frac{b_{r} a_{1 s}}{a_{r s}}$ \\
\hline$\vdots$ & & $\vdots$ & & & $\vdots$ & & $\vdots$ & $\vdots$ & $\vdots$ \\
\hline$\frac{a_{r 1}}{a_{r s}}$ & $\ldots$ & 1 & $\frac{a_{r n}}{a_{r s}}$ & & 0 & $\ldots$ & $\frac{1}{a_{r s}}$ & 0 & $\frac{b_{r}}{a_{r s}}$ \\
\hline$\vdots$ & & $\vdots$ & & & $\vdots$ & & $\vdots$ & $\vdots$ & $\vdots$ \\
\hline$a_{m 1}-\frac{a_{r 1} a_{m s}}{a_{r s}}$ & $\ldots$ & 0 & $\ldots a_{m n}-\frac{a_{r n} a_{m s}}{a_{r s}}$ & & 0 & $\ldots$ & $-\frac{a_{m s}}{a_{r s}}$ & 1 & $b_{m}-\frac{b_{r} a_{m s}}{a_{r s}}$ \\
\hline$c_{1}-\frac{a_{r 1} c_{s}}{a_{r s}}$ & & 0 & $. \quad c_{n}-\frac{a_{r n} c_{s}}{a_{r s}}$ & & 0 & $\cdots$ & $-\frac{c_{s}}{a_{r s}} \quad \ldots$ & 0 & $Z(x)-\frac{b_{r} c_{s}}{a_{r s}}$ \\
\hline
\end{tabular}

Tabela 3.3: Tabela final depois de realizar as operações de pivotamento 
O seguinte fluxograma mostra as iterações do algoritmo simplex

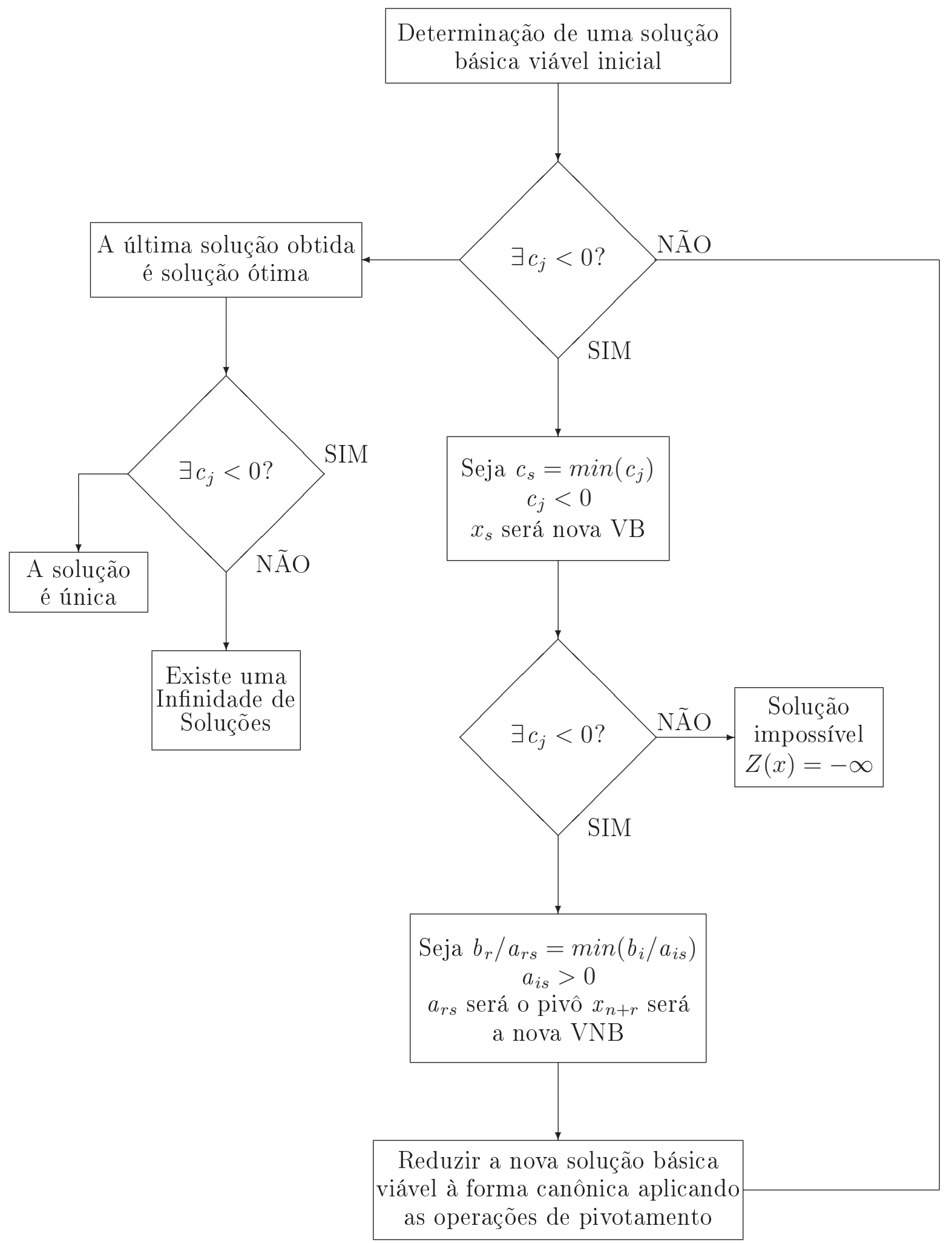

Figura 3.10: Fluxograma do algoritmo Simplex (BREGALDA; OLIVEIRA; BORNSTEIN, 1983) 


\subsection{Matriz de Sensibilidade}

Um domínio $\Omega$ que apresenta uma distribuição não-uniforme de resistividade $\rho$ é discretizado utilizando MEF como foi apresentado na seção 3.1.2. Sob estas hipóteses, pode-se para $\boldsymbol{v}_{j}$ (vetor de potenciais nos nós para cada padrão de corrente), $j=1, \ldots, p$ ( $p$ é o número de padrões de corrente) definir a transformação nãolinear $\boldsymbol{h}_{j}:(\boldsymbol{\rho}, \boldsymbol{I}) \longrightarrow \boldsymbol{v}_{j}$. Partindo da equação (3.12) pode-se escrever a seguinte relação

$$
\boldsymbol{v}_{j}(\boldsymbol{\rho})=\boldsymbol{h}_{j}(\boldsymbol{\rho}) \quad \operatorname{com} \quad \boldsymbol{h}_{j}(\boldsymbol{\rho})=\mathbf{Y}(\boldsymbol{\rho})^{-1} \boldsymbol{I}_{j}
$$

onde $\boldsymbol{I}_{j} \in \Re^{m}$ é o j-ésimo padrão de corrente injetado.

Da equação (3.28), conclui-se que os vetores de potenciais elétricos nodais são funções não-lineares da distribuição de resistividade no domínio. Tomando-se uma aproximação por série de Taylor e truncando a série a partir do termo linear em torno de uma distribuição $\boldsymbol{\rho}_{0}$ arbitrária, segundo as condições do particular problema em estudo ${ }^{7}$, obtém-se

$$
\boldsymbol{v}_{j}(\boldsymbol{\rho}) \approx \boldsymbol{v}_{j}\left(\boldsymbol{\rho}_{0}\right)+\frac{\left.\partial \boldsymbol{h}_{j} \mid \boldsymbol{\rho}_{0}\right)}{\partial \boldsymbol{\rho}}\left[\boldsymbol{\rho}-\boldsymbol{\rho}_{0}\right]
$$

Utilizando a equação (3.28) na equação (3.29) fica

$$
\boldsymbol{v}_{j}(\boldsymbol{\rho})=\boldsymbol{v}_{j}\left(\boldsymbol{\rho}_{0}\right)+\frac{\left.\partial\left([\mathbf{Y}(\boldsymbol{\rho})]^{-1} \boldsymbol{I}_{j}\right)\right|_{\left(\boldsymbol{\rho}_{0}\right)}}{\partial \boldsymbol{\rho}}\left[\boldsymbol{\rho}-\boldsymbol{\rho}_{0}\right]
$$

Da álgebra linear, pode-se escrever que

$$
\frac{\partial A^{-1}}{\partial \theta}=-A^{-1} \frac{\partial A}{\partial \theta} A^{-1}
$$

Com a expressão (3.31) podemos calcular a derivada parcial da transformação $\boldsymbol{h}_{j}$ com respeito a resistividade de cada elemento por

$$
\boldsymbol{H}_{j}\left(\rho_{0}\right)=\frac{\partial \boldsymbol{h}_{j} \mid{ }_{\left(\rho_{0}\right)}}{\partial \rho}=-\mathbf{Y}^{-1} \frac{\partial \mathbf{Y}}{\partial \rho} \mathbf{Y}^{-1} \boldsymbol{I}_{j}
$$

Com isso, a equação (3.30) pode ser escrita como

$$
\underbrace{\left[\boldsymbol{v}_{j}(\boldsymbol{\rho})-\boldsymbol{v}_{j}\left(\boldsymbol{\rho}_{0}\right)\right]}_{\Delta \mathbf{V}}=\boldsymbol{H}_{j} \underbrace{\left(\boldsymbol{\rho}-\boldsymbol{\rho}_{0}\right)}_{\Delta \boldsymbol{\rho}}
$$

onde $\boldsymbol{H}_{j} \in \Re^{p \times n}$ é chamada matriz de sensibilidade.

\footnotetext{
${ }^{7}$ Para o caso da cuba e o objeto, $\rho_{0}$ é o valor de resistividade da solução salina. Para o caso do tórax humano, $\boldsymbol{\rho}_{0}$ é assumido segundo valores encontrados na literatura (ZHANG; PATTERSON, 2006)
} 


\subsubsection{Abordagem do Problema de Imagens de Variação de Resistividade Utilizando Matriz de Sensibilidade}

Para resolver o problema inverso e mal condicionado, são necessárias algumas regularizações para melhorar a condição da matriz a ser invertida.

Define-se o seguinte indice de desempenho ${ }^{8}$

$$
I P=\frac{1}{2}\left(\left.\boldsymbol{H}_{j}\right|_{\rho_{0}} \Delta \boldsymbol{\rho}-\Delta \mathbf{V}_{m}\right)^{T} \mathbf{R}^{T} \mathbf{R}\left(\left.\boldsymbol{H}_{j}\right|_{\rho_{0}} \Delta \boldsymbol{\rho}-\Delta \mathbf{V}_{m}\right)+\frac{\alpha}{2} \Delta \boldsymbol{\rho}^{T} \mathbf{F}^{T} \mathbf{F} \Delta \boldsymbol{\rho}
$$

$\boldsymbol{H}_{j} \longrightarrow$ Matriz de Sensibilidade

$\mathbf{R} \longrightarrow$ Matriz que define quais medidas serão efetivamente utilizadas

$\alpha \longrightarrow$ Parâmetro de regularização

$\Delta \mathbf{V}_{m} \longrightarrow$ Diferença de potencial entre o potencial com objeto $V_{1}$

e o potencial do basal $V_{0}$

$\Delta \boldsymbol{\rho} \longrightarrow$ Diferença de resistividades entre a resistividade $\rho_{1}$ associada

ao potencial $V_{1}$ e a resistividade genérica $\rho_{0}$

$\mathbf{F} \longrightarrow$ Matriz filtro passa altas freqüências espaciais

espaciais no gráfico de resistividade.

A construção do filtro passa Altas freqüências é detalhado em (GONZALEZ; WOODS, 2002). Derivando a equação (3.34) é igualando a zero

$$
\frac{\partial I P}{\partial \Delta \boldsymbol{\rho}}=\left.0 \Longrightarrow \mathbf{H}_{j}\right|_{\boldsymbol{\rho}_{0}} ^{T} \mathbf{R}^{T} \mathbf{R}\left(\Delta \boldsymbol{\rho}-\Delta \mathbf{V}_{m}\right)+\alpha \mathbf{F}^{T} \mathbf{F} \Delta \boldsymbol{\rho}=0
$$

Agrupando termos com $\Delta \rho$

$$
\left(\left.\left.\mathbf{H}_{j}\right|_{\boldsymbol{\rho}_{0}} ^{T} \mathbf{R}^{T} \mathbf{R} \mathbf{H}_{j}\right|_{\boldsymbol{\rho}_{0}}+\alpha \mathbf{F}^{T} \mathbf{F}\right) \Delta \boldsymbol{\rho}=\left.\mathbf{H}_{j}\right|_{\boldsymbol{\rho}_{0}} ^{T} \mathbf{R}^{T} \mathbf{R} \Delta \mathbf{V}_{m}
$$

A equação (3.36) é conhecida como método da matriz de sensibilidade para a obtenção de imagens de variação de resistividade

Examinando a equação (3.36), representa um sistema linear na forma $\mathbf{A x}=\mathbf{b}$,

\footnotetext{
${ }^{8} \mathrm{O}$ índice de desempenho foi gerado utilizando o critério de Bolza da teoria de controle ótimo, o leitor pode consultar (BRYSON; HO, 1966) e (CITRON, 1969) para encontrar informação mais detalhada
} 
fazendo uma comparação é possível identificar a matriz $\mathbf{A}$ e os vetores $\mathbf{x}$ e $\mathbf{b}$

$$
\underbrace{\left(\left.\left.\mathbf{H}_{j}\right|_{\boldsymbol{\rho}_{0}} ^{T} \mathbf{R}^{T} \mathbf{R} \mathbf{H}_{j}\right|_{\boldsymbol{\rho}_{0}}+\alpha \mathbf{F}^{T} \mathbf{F}\right)}_{\mathbf{A}} \underbrace{\Delta \boldsymbol{\rho}}_{\mathbf{x}}=\underbrace{\left.\mathbf{H}_{j}\right|_{\boldsymbol{\rho}_{0}} ^{T} \mathbf{R}^{T} \mathbf{R} \Delta \mathbf{V}_{m}}_{\mathbf{b}}
$$

Aplicando o procedimento mencionando na sub-seção 3.2.4, obtém-se

$$
\begin{gathered}
\text { Minimizar } \\
(\Delta \rho, \xi)
\end{gathered}
$$

Sujeito a

$$
\begin{aligned}
& \left(\left(\left.\left.\mathbf{H}_{j}\right|_{\boldsymbol{\rho}_{0}} ^{T} \mathbf{R}^{T} \mathbf{R} \mathbf{H}_{j}\right|_{\boldsymbol{\rho}_{0}}+\alpha \mathbf{F}^{T} \mathbf{F}\right) \Delta \boldsymbol{\rho}-\left.\mathbf{H}_{j}\right|_{\boldsymbol{\rho}_{0}} ^{T} \mathbf{R}^{T} \mathbf{R} \Delta \mathbf{V}_{m}\right)+\xi \mathbf{e}=0 \\
& \Delta \boldsymbol{\rho}_{\min } \leq \Delta \boldsymbol{\rho} \leq \Delta \boldsymbol{\rho}_{\max } \\
& \quad 0 \leq \xi \leq \epsilon
\end{aligned}
$$

Onde

$$
\mathbf{e}=\left(\left(\left.\left.\mathbf{H}_{j}\right|_{\boldsymbol{\rho}_{0}} ^{T} \mathbf{R}^{T} \mathbf{R} \mathbf{H}_{j}\right|_{\boldsymbol{\rho}_{0}}+\alpha \mathbf{F}^{T} \mathbf{F}\right) \Delta \boldsymbol{\rho}_{a}\right)-\left(\left.\mathbf{H}_{j}\right|_{\boldsymbol{\rho}_{0}} ^{T} \mathbf{R}^{T} \mathbf{R} \Delta \mathbf{V}_{m}\right)
$$

A imposição de limites em $\Delta \rho$ na equação (3.38) introduz informação a priori. Por exemplo o valor de resistividade deve ser positivo e para o limite superior é imposto o valor máximo de resistividade que pode ser encontrado. Para aplicações médicas, as resistividades dentro do tórax, são valores positivos que variam desde $4 \Omega m$ para o coração (músculo) até $1 \times 10^{5} \Omega m$ para o ar presente nos pulmões (ZHANG; PATTERSON, 2006). Partindo desta informação, é possível impor os limites nas variáveis para obter imagens médicas.

\subsection{Método Newton-Raphson}

Newton-Raphson é um método numérico utilizado na solução de sistemas nãolineares que apresentam a forma $f_{i}=0$ para $i=1, \ldots, n$ (ARUCA, 2002). Para o caso no qual $f(x)$ é uma função de uma única variável $x$ e existe sua primeira derivada, geometricamente o método consiste em estender a reta tangente que passa pelo ponto $f\left(x_{n}\right)$ até cruzar o eixo das abcissas, onde um novo ponto $x_{n+1}$ é determinado e continua-se iterativamente até encontrar o ponto de mínimo. 


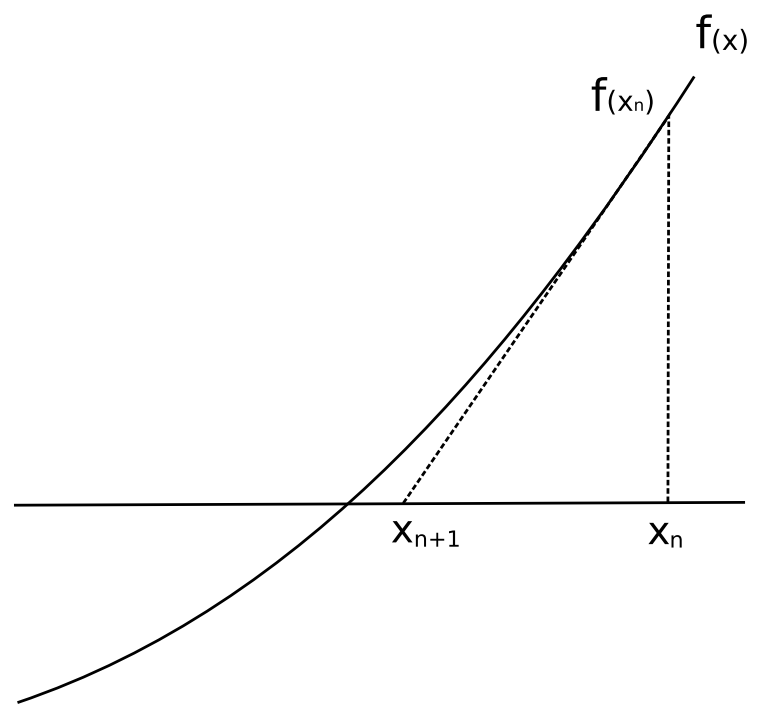

Figura 3.11: O ponto $x_{n+1}$ denota a aproximação do método Newton-Raphson ao zero da função $f(x)$.

Para o problema típico de nosso interesse em que $\mathrm{N}$ funções são igualadas a zero, nas proximidades da solução cada função pode ser aproximada por uma série de Taylor truncada

$$
F\left(x_{k}+p\right) \approx F(k)+g_{k}^{T} p+\frac{1}{2} p^{T} G_{k} p
$$

onde $p$ é o passo para chegar ao mínimo da função, $g_{k}$ é um vetor de constantes e $G_{k}$ é a matriz Hessiana de F. O mínimo do lado direto da equação 3.39 será atingido se $p_{k}$ for o mínimo da função quadrática

$$
\Phi_{p}=g_{k}^{T} p+\frac{1}{2} p^{T} G_{k} p
$$

A função $\Phi$ tem um ponto estacionário somente se existe um ponto $x^{*}$ onde o vetor gradiente se anule, isto é, deve-se cumprir que $\nabla \Phi\left(x^{*}\right)=G x^{*}+g=0$ (GILL; MURRAY; WRIGHT, 1986). Conseqüentemente, um ponto estacionário $p_{k}$ deve satisfazer o seguinte sistema de equações lineares:

$$
G_{k} p_{k}=-g_{k}
$$

O algoritmo de minimização no qual $p_{k}$ é encontrado como mostrado na equação 3.41 é chamado Método Newton-Raphson, e a solução da equação 3.41 é chamado de direção de Newton-Raphson. 


\subsubsection{Método Gauss-Newton}

Em um grande número de problemas práticos, a função $F(x)$ é uma soma dos quadrados de funções não-lineares

$$
F(x)=\frac{1}{2} \sum_{i=1}^{m} f_{i}(x)^{2}=\frac{1}{2}\|\mathbf{f}(x)\|_{2}^{2}
$$

Onde a i-componente do vetor $\boldsymbol{f}(\boldsymbol{x})$ é a função $f_{i}(x)$ e $\|\mathbf{f}(x)\|$ é o resíduo de $x$. O Gauss-Newton é um método utilizado na minimização de sistemas não-lineares e utiliza a informação da primeira e segunda derivada das funções $f_{i}(x)$. Seja $\mathbf{J}(x)$ a matriz Jacobiana da função $\boldsymbol{f}(\boldsymbol{x})$ e $\mathbf{G}_{i}(x)$ a matriz Hessiana de $f_{i}(x)$. Então

$$
\begin{aligned}
& g(x)=\mathbf{J}(x)^{T} f(x) \\
& G(x)=\mathbf{J}(x)^{T} \mathbf{J}(x)+\mathbf{Q}(x),
\end{aligned}
$$

Onde $\mathbf{Q}(x)=\sum_{i=1}^{m} f_{i}(x) \mathbf{G}_{i}(x)$. Da equação 3.44 observa-se que a matriz Hessiana da função objetivo é uma combinação de informação de primeira e segunda ordem. Os métodos de mínimos quadrados são tipicamente baseados na premissa que eventualmente o termo de primeira ordem $\mathbf{J}^{T}(x) \mathbf{J}(x)$ da equação 3.44 dominará o termo de segunda ordem Q $(x)$ (GILL; MURRAY; WRIGHT, 1986). Utilizando as equações 3.43 e 3.44 na equação 3.41 do Método Newton-Raphson

$$
\left(\mathbf{J}_{k}^{T} \mathbf{J}_{k}+\mathbf{Q}_{k}\right) \mathbf{p}_{k}=-\mathbf{J}_{k}^{T} \mathbf{f}_{k}
$$

Sendo $\mathbf{p}_{k}$ a solução da equação 3.45 e $\mathbf{x}_{k}$ representa a estimativa atual da solução. Se $\left\|\mathbf{f}_{k}\right\|$ tende a zero quando $\mathbf{x}_{k}$ chega no ponto ótimo então a matriz $\mathbf{Q}_{k}$ também tende a zero. Para $\mathbf{x}_{k}$ próximo da solução, a equação 3.45 pode ser escrita

$$
\mathbf{J}_{k}^{T} \mathbf{J}_{k} \mathbf{p}_{k}=-\mathbf{J}_{k}^{T} \mathbf{f}_{k}
$$

O vetor que resolve a equação 3.46 é chamado direção de Gauss-Newton e o método no qual o vetor é utilizado como direção de busca é conhecido como Método de Gauss-Newton.

\subsubsection{Abordagem do Problema de Imagens Absolutas de Resistividade Utilizando Método de Gauss-Newton}

Para determinar o vetor de distribuição de resistividade $\boldsymbol{\rho}_{i} \in \Re^{n}$ partindo do potencial medido, o problema inverso é escrito

$$
\boldsymbol{\rho}^{*}=\min _{\boldsymbol{\rho}}\left\{\left\|\mathbf{V}_{m}-\mathbf{V}_{c}\right\|_{2}^{2}+\alpha\left\|\boldsymbol{\rho}^{T} \mathbf{F}^{T} \mathbf{F} \boldsymbol{\rho}\right\|\right\}
$$


onde $\mathbf{V}_{m} \in \Re^{e^{2}}$ é o vetor dos potenciais medidos, $\mathbf{V}_{c} \in \Re e^{2}$ é o vetor dos potenciais obtidos do modelo, $\alpha$ é um parâmetro de regularização e $\mathbf{F}$ é uma matriz filtro passa altas freqüências espaciais. Para uma iteração $k$ do Método Gauss-Newton, o método atualiza o vetor $\boldsymbol{\rho}_{k}$ para $\boldsymbol{\rho}_{k+1}$ de acordo com

$$
\boldsymbol{\rho}_{k+1}=\boldsymbol{\rho}_{k}+\boldsymbol{\delta} \boldsymbol{\rho}_{k}
$$

onde

$$
\boldsymbol{\delta} \boldsymbol{\rho}_{k}=\left(\mathbf{J}_{\rho}^{T} \mathbf{J}_{\rho}+\alpha \mathbf{F}^{T} \mathbf{F}\right)^{-1}\left(\mathbf{J}_{\rho}^{T}\left(\mathbf{V}_{m}-\mathbf{V}_{c}\right)-\alpha \mathbf{F}^{T} \mathbf{F} \boldsymbol{\rho}_{k}\right)
$$

Comparando as equações $3.43,3.44$ e 3.46 com a equação 3.49 pode-se estabelecer as relações

$$
\begin{aligned}
& \mathbf{G}(\mathbf{x})=\mathbf{J}_{\rho}^{T} \mathbf{J}_{\rho}+\alpha \mathbf{F}^{T} \mathbf{F} \\
& \mathbf{g}(\mathbf{x})=\mathbf{J}_{\rho}^{T}\left(\mathbf{V}_{m}-\mathbf{V}_{c}\right)-\alpha \mathbf{F}^{T} \mathbf{F} \boldsymbol{\rho}_{k} \\
& \mathbf{p}_{\mathbf{k}}=\boldsymbol{\delta} \boldsymbol{\rho}_{k}
\end{aligned}
$$

A matriz Jacobiana em cada iteração $k$ é

$$
\mathbf{J}_{k}=-\mathbf{Y}_{k}^{-1} \frac{\partial \mathbf{Y}_{k}}{\partial \boldsymbol{\rho}_{k}} \mathbf{Y}_{k}^{-1} \boldsymbol{I}
$$

Olhando para a equação 3.49 encontra-se um sistema linear na forma $\mathbf{x}=\mathbf{A}^{-\mathbf{1}} \mathbf{b}$ o qual pode-ser resolvido com a formulação apresentada na seção 3.2.4

Minimizar $\quad \xi$

$(\boldsymbol{\delta} \rho, \xi)$

Sujeito a

$$
\begin{gathered}
\left(\left(\mathbf{J}_{\rho}^{T} \mathbf{J}_{\rho}+\alpha \mathbf{F}^{T} \mathbf{F}\right) \boldsymbol{\delta} \boldsymbol{\rho}_{k}-\left(\mathbf{J}_{\rho}^{T}\left(\mathbf{V}_{m}-\mathbf{V}_{c}\right)-\alpha \mathbf{F}^{T} \mathbf{F} \boldsymbol{\rho}_{k}\right)\right)+\xi \mathbf{e}=0 \\
\boldsymbol{\delta} \boldsymbol{\rho}_{k}^{\text {inf }} \leq \boldsymbol{\delta} \boldsymbol{\rho}_{k} \leq \boldsymbol{\delta} \boldsymbol{\rho}_{k}^{\text {sup }} \\
0 \leq \xi \leq \epsilon
\end{gathered}
$$

Onde

$\mathbf{e}=\left(\left(\mathbf{J}_{\rho}^{T} \mathbf{J}_{\rho}+\alpha \mathbf{F}^{T} \mathbf{F}\right) \boldsymbol{\delta} \boldsymbol{\rho}_{0}\right)-\left(\left(\mathbf{J}_{\rho}^{T}\left(\mathbf{V}_{m}-\mathbf{V}_{c}\right)-\alpha \mathbf{F}^{T} \mathbf{F} \boldsymbol{\rho}_{k}\right)\right)$

$\boldsymbol{\delta} \boldsymbol{\rho}_{k}^{\text {inf }} \longrightarrow$ Limite inferior calculado como mostrado na seção 3.3.1

$\boldsymbol{\delta} \boldsymbol{\rho}_{k}^{\text {sup }} \longrightarrow$ Limite superior calculado como mostrado na seção 3.3.1 


\subsubsection{Implementação da RIM na Distribuição de Resistivi- dade}

Para poder implementar a RIM na determinação do $\boldsymbol{\delta} \boldsymbol{\rho}$ é preciso acrescentar a matriz de restrições $\mathbf{S}$ mostrada na equação 3.16 às restrições de elementos finitos mostradas na equação 3.51. O sistema linear apresentando na seção 3.1.7 é função do vetor de resistividade $\boldsymbol{\rho}$, portanto, é necessário lembrar que para uma iteração $k+1$ do método Gauss-Newton o vetor de resistividade na iteração $k+1$ é igual a

$$
\boldsymbol{\rho}_{k+1}=\boldsymbol{\rho}_{k}+\boldsymbol{\delta} \boldsymbol{\rho}_{k}
$$

Substituindo a equação 3.53 na equação 3.16 obtém-se

$$
[\mathbf{S}]\left\{\boldsymbol{\rho}_{k}+\boldsymbol{\delta} \boldsymbol{\rho}_{k}\right\} \leq\{\mathbf{c}\}
$$

Na equação anterior, o vetor $\boldsymbol{\rho}_{k}$ é conhecido, por tanto

$$
[\mathbf{S}]\left\{\boldsymbol{\delta} \boldsymbol{\rho}_{k}\right\} \leq\{\mathbf{c}\}-[\mathbf{S}]\left\{\boldsymbol{\rho}_{k}\right\}
$$

Com a equação 3.55 em função do $\boldsymbol{\rho}_{k}$, o sistema da equação 3.55 pode ser acrescentado ao sistema linear de restrições de elementos finitos apresentado na equação 3.51. Finalmente o problema a ser resolvido quando é implementada a RIM torna-se

$$
\begin{gathered}
\text { Minimizar } \\
(\boldsymbol{\delta} \rho, \xi)
\end{gathered}
$$

Sujeito a

$$
\begin{gathered}
\left(\left(\mathbf{J}_{\rho}^{T} \mathbf{J}_{\rho}+\alpha \mathbf{F}^{T} \mathbf{F}\right) \boldsymbol{\delta} \boldsymbol{\rho}_{k}-\left(\mathbf{J}_{\rho}^{T}\left(V_{m}-V_{c}\right)-\alpha \mathbf{F}^{T} \mathbf{F} \boldsymbol{\rho}_{k}\right)\right)+\xi \mathbf{e}=0 \\
{[\mathbf{S}]\left\{\boldsymbol{\delta} \boldsymbol{\rho}_{k}\right\} \leq\{\mathbf{c}\}-[\mathbf{S}]\left\{\boldsymbol{\rho}_{k}\right\}} \\
\boldsymbol{\delta} \boldsymbol{\rho}_{k}^{\text {inf }} \leq \boldsymbol{\delta} \boldsymbol{\rho}_{k} \leq \boldsymbol{\delta} \boldsymbol{\rho}_{k}^{\text {sup }} \\
0 \leq \xi \leq \epsilon
\end{gathered}
$$

Onde

$\mathbf{e}=\left(\left(\mathbf{J}_{\rho}^{T} \mathbf{J}_{\rho}+\alpha \mathbf{F}^{T} \mathbf{F}\right) \boldsymbol{\delta} \boldsymbol{\rho}_{0}\right)-\left(\left(\mathbf{J}_{\rho}^{T}\left(V_{m}-V_{c}\right)-\alpha \mathbf{F}^{T} \mathbf{F} \boldsymbol{\rho}_{k}\right)\right)$

$\boldsymbol{\delta} \boldsymbol{\rho}_{k}^{\text {inf }} \longrightarrow$ Limite inferior móvel, ver seção 3.3.1

$\boldsymbol{\delta} \boldsymbol{\rho}_{k}^{\text {sup }} \longrightarrow$ Limite superior móvel, ver seção 3.3.1 


\section{Metodologia}

O problema de estimação pode ser formulado como um problema de otimização e pode ser resolvido de diversas formas. Uma comparação entre dois métodos pode revelar vantagens ${ }^{1}$, o efeito da propagação de erros numéricos, a estabilidade em condições limites e modificações para torná-los mais efetivos.

As características de PL que podem ser de valor na estimação de imagens da TIE são basicamente duas, PL propaga menos ruído numérico e é fácil impor restrições nas variáveis restringindo o espaço solução ao espaço clinicamente significativo.

O algoritmo de PL apresentado neste projeto vai ser testado através de dois tipos de testes. Foram utilizadas subrotinas para o cálculo numérico escritas em C, que integram a biblioteca de funções "GNU Linear Programming (GLPK)"e que são distribuídas sobre licença GPL pela "Free Software Foundation". O programa foi compilado usando o compilador gec, no sistema operacional GNULinux. O procedimento para PL na TIE, ou o procedimento para utilizar PL para resolver sistemas lineares, está detalhado na subseção 3.2.4.

\section{$4.1 \quad$ Testes}

Para avaliar o desempenho de PL, dois testes vão ser implementados, o primeiro tipo de teste tem como objetivo demonstrar que PL gera menos propagação de erro numérico no momento de inverter matrizes comparado com o método LU Decomposition e o segundo tipo de teste procura aproveitar a vantagem de poder impor restrições nas variáveis implementando PL no problema de imagens de variação de resistividade utilizando Matriz de Sensibilidade, e em imagens absolutas, utilizando o Método Gauss-Newton.

As malhas de elementos finitos utilizadas nos dois diferentes testes foram

\footnotetext{
${ }^{1}$ No caso de estudo foi comparado PL com o método que atualmente é implementado para resolver sistemas lineares
} 
geradas com o Programa Gmsh, da Free Software Foundation, em plataforma GNU-Linux.

No segundo tipo de teste, quando as imagens obtidas são de variação de resistividade é necessário ter dados de potenciais elétricos provenientes de um fantoma com solução salina (basal) e com solução salina com um objeto ou elemento da malha (perturbado). Quando as imagens são absolutas só é necessário ter dados com objeto. As medidas de potenciais elétricos, quanto basal como perturbado, são geradas com ajuda de uma simulação numérica ou de uma bancada experimental.

Dados de simulação numérica. O problema direto que gera os dados simulados consiste num domínio circular, onde a distribuição de resistividade é conhecida a priori. Para gerar os dados de potenciais elétricos simulados pode-se considerar um domínio circular homogêneo, isto é, com distribuição de resistividade constante. Para obter dados simulados do fantoma perturbado, escolhe-se um elemento qualquer da malha de elementos finitos e atribui-se um valor de resistividade diferente do basal. Nas figuras 4.1(a) e 4.1(b) é mostrado a forma como é posicionado o objeto para gerar dados numéricos em duas diferentes posições, assim como as dimensões da cuba e do objeto

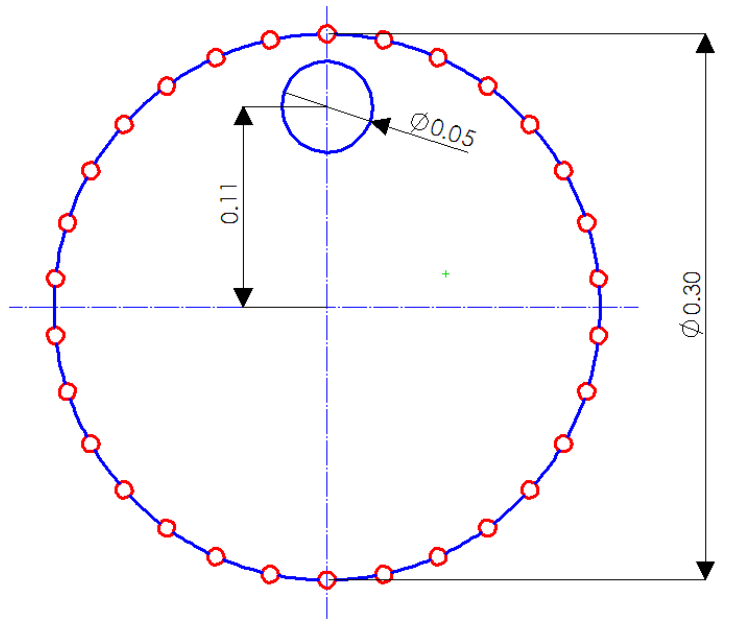

(a)

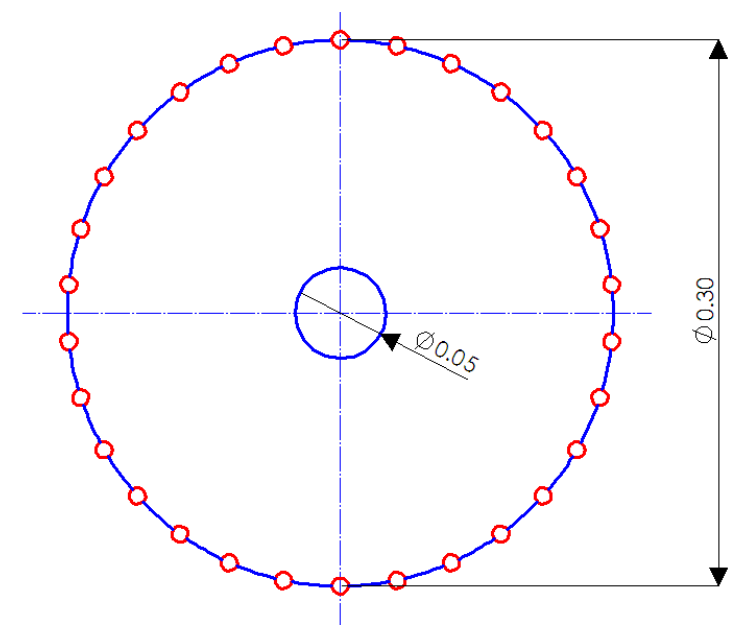

(b)

Figura 4.1: Objeto posicionado (a) na borda (b) no centro

Dados da bancada experimental. Os dados da bancada experimental são gerados injetando corrente nos eletrodos, os quais estão alocados num domínio circular chamado cuba, dentro da cuba encontra-se uma solução salina e um objeto de acrílico ou objetos de $\operatorname{agar}^{2}$ simulando o coração e o pulmão. Para o caso do 
objeto de acrílico, o potencial elétrico vai ser medido em duas posições diferentes como é mostrado nas figuras $4.2(\mathrm{a})$ e $4.2(\mathrm{~b})$.

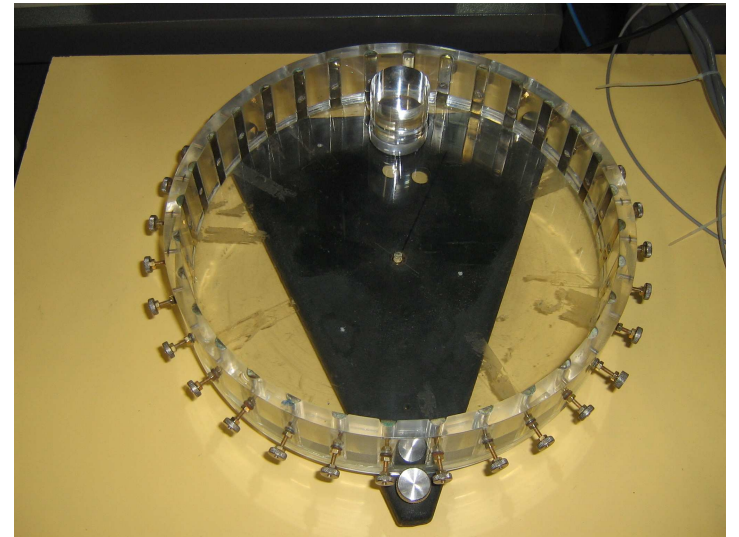

(a)

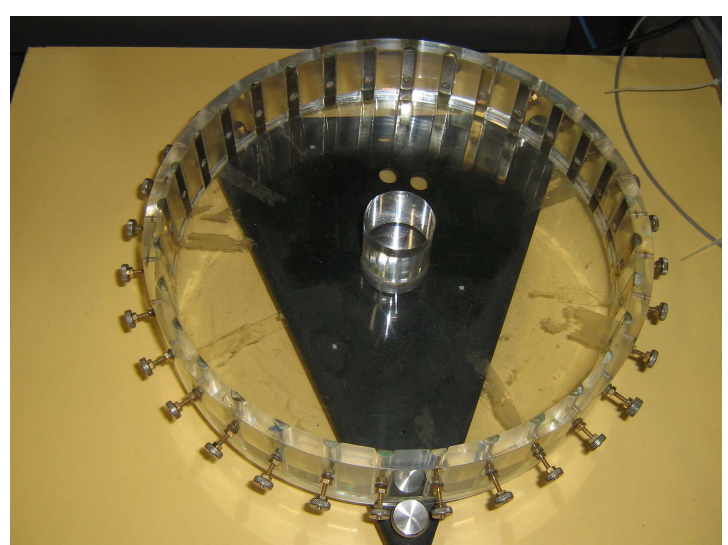

(b)

Figura 4.2: Objeto posicionado (a) na borda (b) no centro

As figuras 4.3(a) e 4.3(b) mostram os objetos feitos de agar, as resistividades dos objetos que estão simulando os pulmões e o coração foram determinadas com o condutivímetro NT-CVP da Nova Técnica.

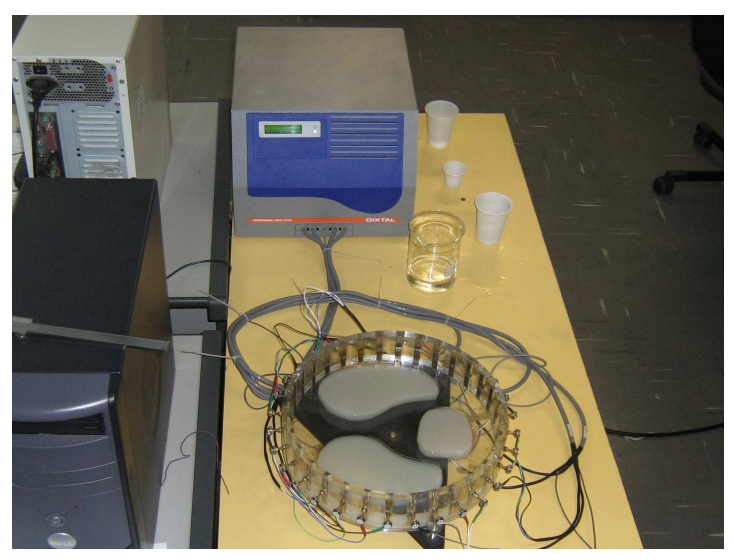

(a)

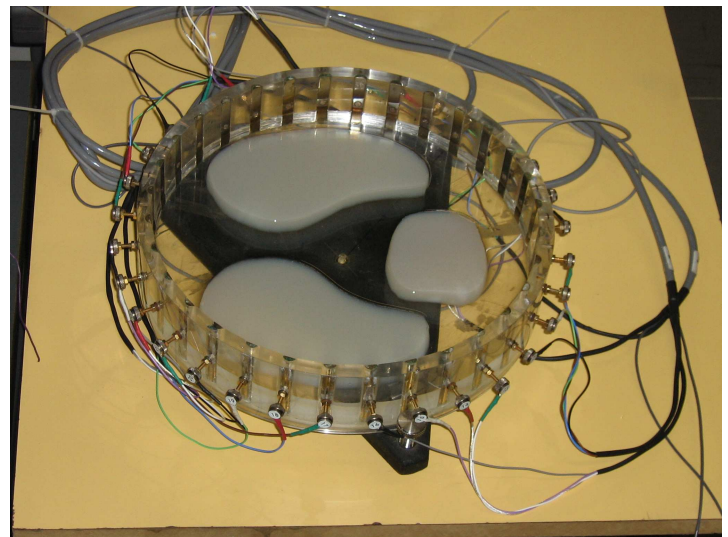

(b)

Figura 4.3: Objetos de agar simulando a geometria do pulmão e coração

Dados do tórax humano. Os dados foram coletados dentro do projeto temático FAPESP número 01/05303 - 4 (01/07/2002 até 31/08/2008). Foi injetada uma corrente senoidal alternada de $4,33 \mathrm{~mA}$ pico a uma freqüência de $125 \mathrm{kHz}$ com o tomógrafo de impedância elétrica mostrado na figura 4.15. O padrão de injeção de corrente foi pula três eletrodos mostrado na figura 3.5(b) com um total de 32 eletrodos alocados ao redor do tórax como mostrado nas figuras 4.4(a) e 4.4(b), as medidas de potencial elétrico foram coletadas para 1000 frames e com o indivíduo retendo ar nos pulmões (inspiração). 


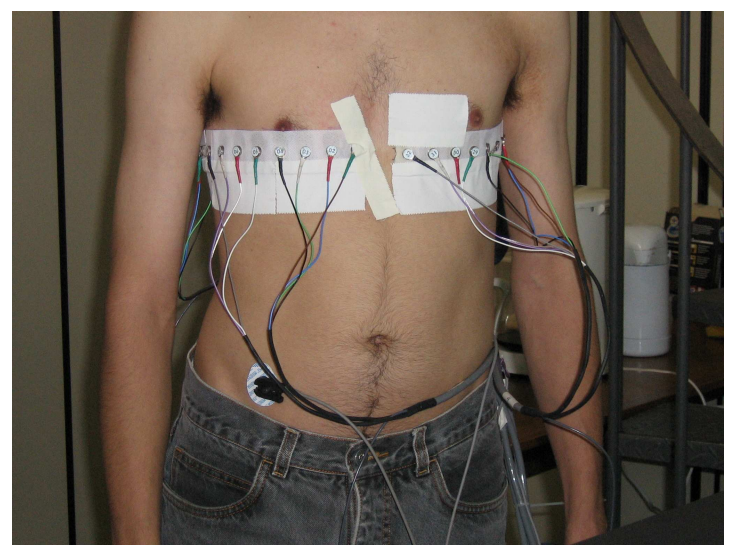

(a)

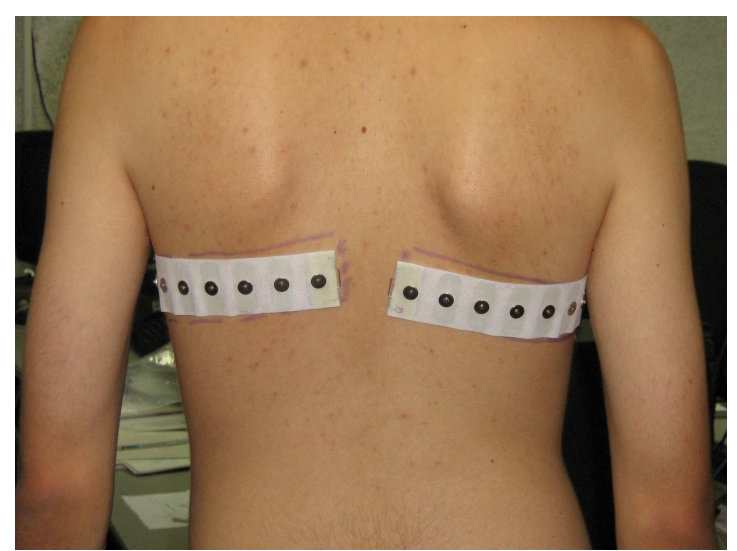

(b)

Figura 4.4: Posicionamento dos eletrodos para gerar imagens do tórax humano

\subsubsection{Diminuição do Erro Numérico via PL}

Nesta seção testa-se a hipóteses de que ao resolver um sistema linear usando PL a propagação de erro numérico é menor do que quando se resolve o sistema linear via LU Decomposition. Da álgebra de matrizes

$$
\mathbf{A A}^{-1}=\mathbf{I}
$$

ou reescrevendo

$$
\mathbf{A A}^{-1}-\mathbf{I}=0
$$

O erro numérico na inversão da matriz $\mathbf{A}$ provoca

$$
\mathbf{A A}^{-1}-\mathbf{I}=\mathbf{E}
$$

onde $\mathbf{E}$ é uma matriz com valores próximos de zero. Uma norma da matriz $\mathbf{E}$ é uma medida da qualidade da inversão da matriz A. Seja $\epsilon=\|\mathbf{E}\|$ um escalar que quanto menor, menor será a propagação de erro na inversão de $\mathbf{A}$.

O sistema linear que desejamos resolver neste trabalho está definido na equação 3.37. Desta forma, a matriz do sistema que iremos utilizar no lugar da matriz A é

$$
\left.\left.\mathbf{H}_{j}\right|_{\rho_{0}} ^{T} \mathbf{R}^{T} \mathbf{R} \mathbf{H}_{j}\right|_{\rho_{0}}+\alpha \mathbf{F}^{T} \mathbf{F}
$$


Iremos comparar o valor de $\epsilon$ obtido com PL com o valor de $\epsilon$ obtido utilizando funções para inversão de matrizes da biblioteca MKL ${ }^{3}$ (Intel Kernel Library). O parâmetro de regularização $\alpha$ será diminuido para poder observar o comportamento tanto de PL como da biblioteca da MKL quando a matriz A vai se tornando mal condicionada. ${ }^{4}$

Na figura 4.5 mostra-se a malha utilizada para gerar a matriz $\boldsymbol{H}_{j}$, ou seja matriz $A$ no contexto desta subseção.

\footnotetext{
${ }^{3}$ http : //www.intel.com/cd/software/products/asmo - na/eng/perflib/mkl/index.htm Método de inversão de matrizes LU Decomposition

${ }^{4}$ Para obter a inversa da matriz A utilizando PL, é necessário resolver um número de sistemas lineares igual ao número de elementos finitos da malha, isto é, para cada coluna a determinar da matriz inversa resolve-se um sistema linear. No caso de uma malha de três elementos finitos, o tamanho da matriz $\mathbf{A}$ é de $3 \times 3$ e o número de sistemas lineares que tem-se que resolver para encontrar a matriz inversa $\mathbf{A}^{-1}$ com PL é igual a três.
}

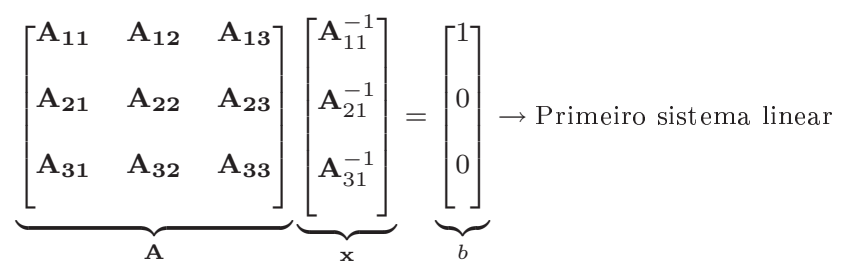

$\left[\begin{array}{lll}\mathbf{A}_{11} & \mathbf{A}_{12} & \mathbf{A}_{13} \\ \mathbf{A}_{21} & \mathbf{A}_{22} & \mathbf{A}_{23} \\ \mathbf{A}_{31} & \mathbf{A}_{32} & \mathbf{A}_{33}\end{array}\right]\left[\begin{array}{l}\mathbf{A}_{12}^{-1} \\ \mathbf{A}_{22}^{-1} \\ \mathbf{A}_{32}^{-1}\end{array}\right]=\left[\begin{array}{l}0 \\ 1 \\ 0\end{array}\right] \rightarrow$ Segundo sistema linear

$$
\left[\begin{array}{lll}
\mathbf{A}_{11} & \mathbf{A}_{12} & \mathbf{A}_{13} \\
\mathbf{A}_{21} & \mathbf{A}_{22} & \mathbf{A}_{23} \\
\mathbf{A}_{31} & \mathbf{A}_{32} & \mathbf{A}_{33}
\end{array}\right]\left[\begin{array}{l}
\mathbf{A}_{13}^{-1} \\
\mathbf{A}_{23}^{-1} \\
\mathbf{A}_{33}^{-1}
\end{array}\right]=\left[\begin{array}{l}
0 \\
0 \\
1
\end{array}\right] \rightarrow \text { Terceiro sistema linear }
$$

O primeiro sistema linear vai determinar a primeira coluna da matriz $\mathbf{A}^{-1}$, o segundo sistema linear vai terminar a segunda coluna da matriz $\mathbf{A}^{-1}$ e o último sistema linear determina a última coluna da matriz $\mathbf{A}^{-1}$. A solução de cada sistema linear é feita com o procedimento apresentado na seção 3.2.4 e mostrado a seguir apenas para o primeiro sistema linear onde o vetor coluna $\mathbf{A}_{i 1}^{-1} \operatorname{com} i=1,2,3$ é chamado de $\mathbf{x}$ e o vetor coluna de componentes $\left[\begin{array}{lll}1 & 0 & 0\end{array}\right]^{T}$ é chamado de $\mathbf{b}$

$$
\begin{gathered}
\text { Minimizar } \quad \xi \\
\text { Sujeito a } \\
\begin{array}{c}
\mathbf{A x}-\mathbf{b}+\xi \mathbf{e}=0 \\
x_{\min } \leq \mathbf{x} \leq x_{\max } \\
0 \leq \xi \leq \epsilon
\end{array}
\end{gathered}
$$

Quando $\xi \approx 0$, o vetor $\mathbf{x}$ satisfaz $\mathbf{A x}=\mathbf{b}$.

Da formulação apresentada, observa-se que PL precisa de limites nas variáveis e o problema a resolver não proporciona informação física para impor ditos limites. Adotam-se limites construídos em torno de cada coluna da matriz inversa de $A$ obtida por LU Decomposition. Portanto, na solução do primeiro sistema linear, resulta o problema de otimização

$$
\begin{aligned}
& \text { Minimizar } \quad \xi \\
& \text { Sujeito a } \\
& \qquad \begin{array}{l}
\mathbf{A x}-\mathbf{b}+\xi \mathbf{e}=0 \\
L I * \mathbf{A}_{i 1}^{L U} \leq \mathbf{x}_{i} \leq L S * \mathbf{A}_{i 1}^{L U} \\
0 \leq \xi \leq \epsilon
\end{array}
\end{aligned}
$$




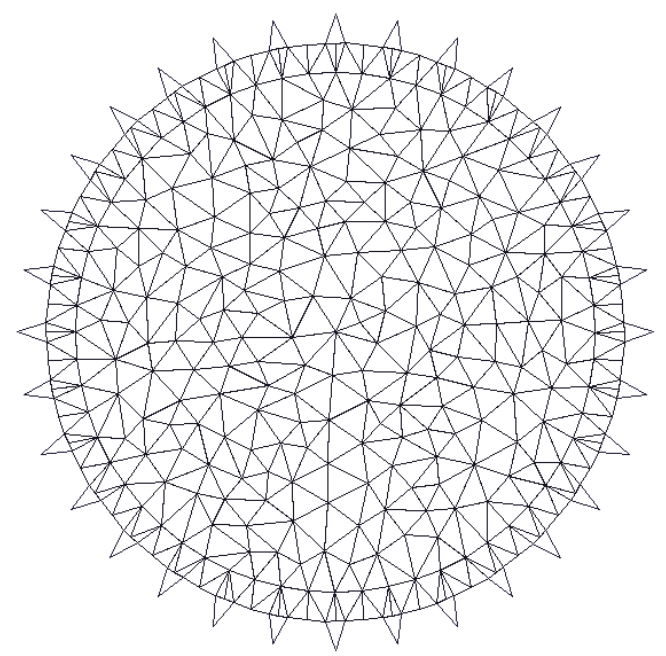

Figura 4.5: Malha composta por 1938 nós, 606 elementos e 30 eletrodos

\subsubsection{PL Comparado com LU Decomposition - Matriz de Sensibilidade}

O objetivo de implementar PL no problema de obtenção de imagens de variação de resistividade é aproveitar uma suposta da diminuição do erro numérico e aproveitar a facilidade de imposição de limites nas variáveis em PL para obter melhorias nas imagens. As imagens obtidas com PL vão ser comparadas com as imagens obtidas com LU Decomposition.

\subsubsection{PL comparado com LU Decomposition - Gauss-Newton}

A implementação de PL no método Gauss-Newton vai ser na solução do sistema linear da equação (3.49). É necessário utilizar programação linear seqüencial (PLS). Em cada iteração do algoritmo Gauss-Newton, novos limites inferiores e superiores serão calculados. O desempenho da PLS será avaliado ao comparar a diferença entre o potencial elétrico medido e o potencial elétrico calculado.

\subsubsection{Dados de Simulação Numérica}

\subsubsection{Dados de Simulação Numérica para Matriz de Sensibilidade}

1. Elemento na borda: foram usadas duas malhas de elementos finitos diferentes para resolver o problema direto e inverso. A malha para o problema direto mostrada na figura 4.6(a) tem 840 nós, 240 elementos e 30 eletrodos; enquanto que a malha para o problema inverso, tem 768 nós, 216 elementos 
e 30 eletrodos como se observa na figura 4.6(b). Assumiu-se uma resistividade de $10 \Omega m$ para todos os elementos, exceto no elemento 175 (ressaltado em vermelho) cuja resistividade adotou-se igual a $50 \Omega \mathrm{m}$.

2. Elemento no centro: as mesmas malhas de elementos finitos das figuras 4.6(a) e 4.6(b) foram usadas novamente para resolver o problema direto e o problema inverso. No problema direto admitiu-se uma resistividade homogênea de $10 \Omega m$ para todos os elementos, exceto para o elemento 217 (ressaltado em laranja) cuja resistividade adotou-se igual $50 \Omega m$.

3. Objeto na borda: foram usadas duas malhas de elementos finitos diferentes para resolver o problema direto e inverso. A malha para o problema direto mostrada na figura 4.7(a) tem 3348 nós, 1076 elementos e 30 eletrodos; enquanto que a malha para o problema inverso, tem 2478 nós, 786 elementos e 30 eletrodos como se observa na figura 4.7(b). Assumiu-se uma resistividade de $10 \Omega m$ para todos os elementos, exceto no objeto cuja resistividade adotou-se igual a $100 \Omega m$.

4. Objeto no centro: A malha do problema direto mostrada na figura 4.8(a) tem 3522 nós, 1134 elementos e 30 eletrodos; enquanto que a malha do problema inverso mostrada na figura 4.8(b) tem 2508 nós, 796 elementos e 30 eletrodos. No problema direto admitiu-se uma resistividade homogênea de $10 \Omega m$ para todos os elementos, exceto para o objeto cuja resistividade adotou-se igual a $100 \Omega m$.

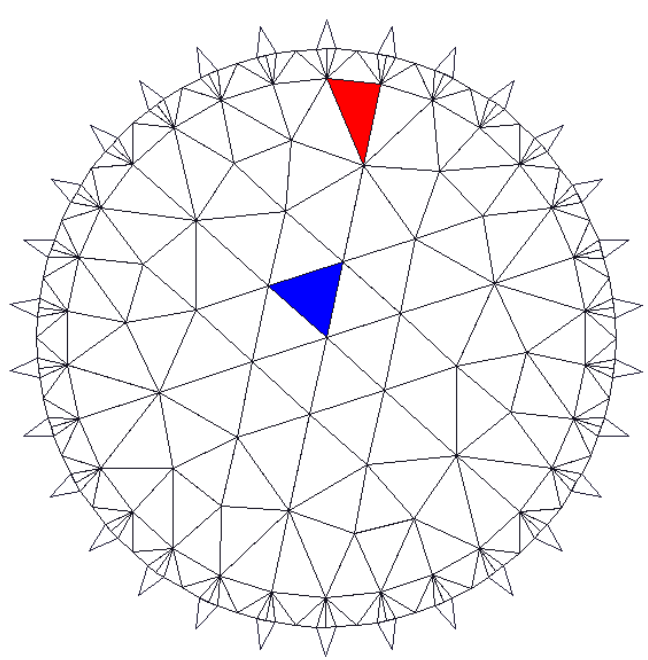

(a)

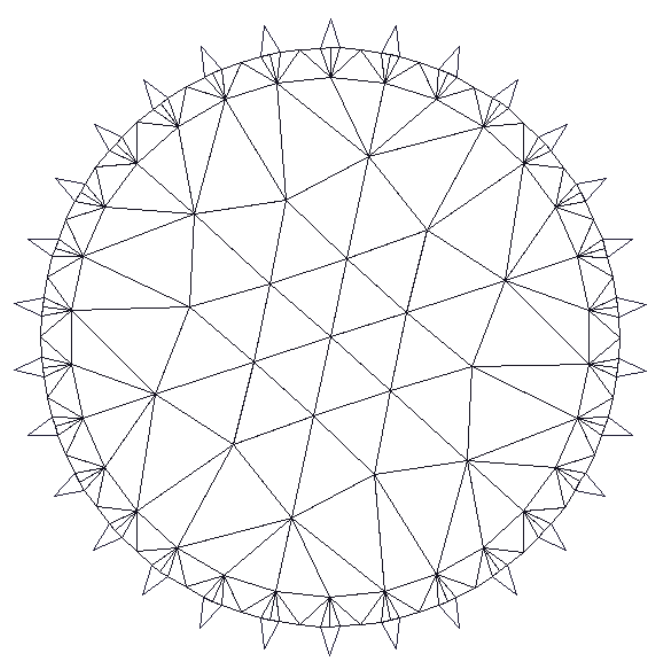

(b)

Figura 4.6: Malhas de elementos finitos para resolver o problema (a) direto e (b) inverso 


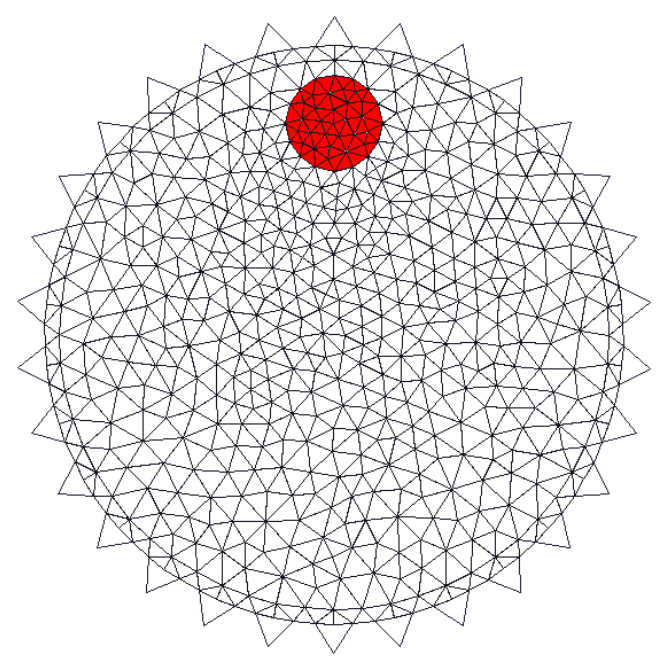

(a)

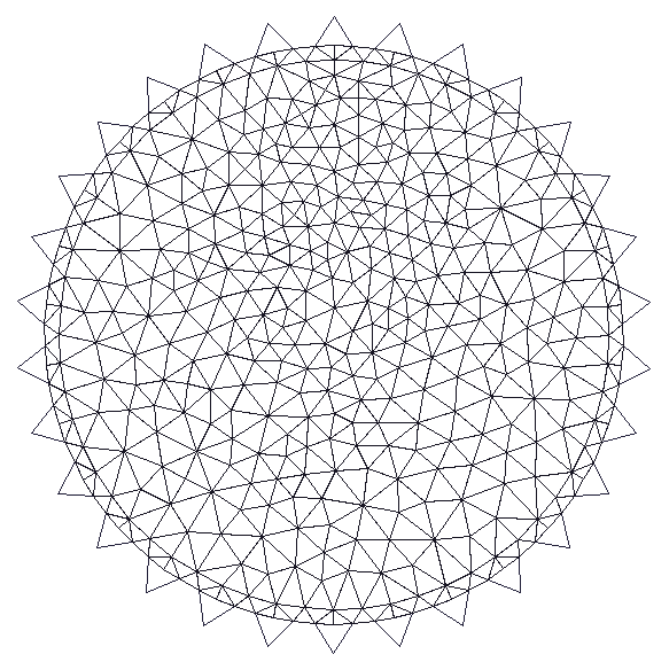

(b)

Figura 4.7: Malhas de elementos finitos para resolver o problema (a) direto e (b) inverso

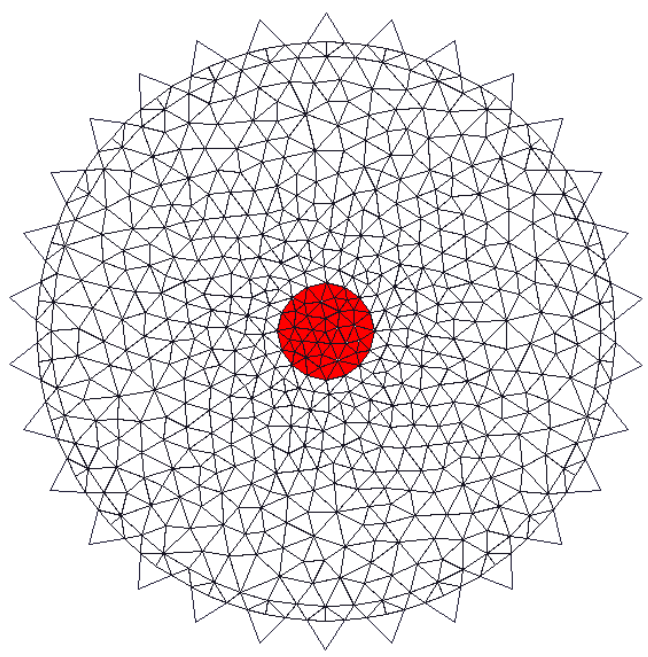

(a)

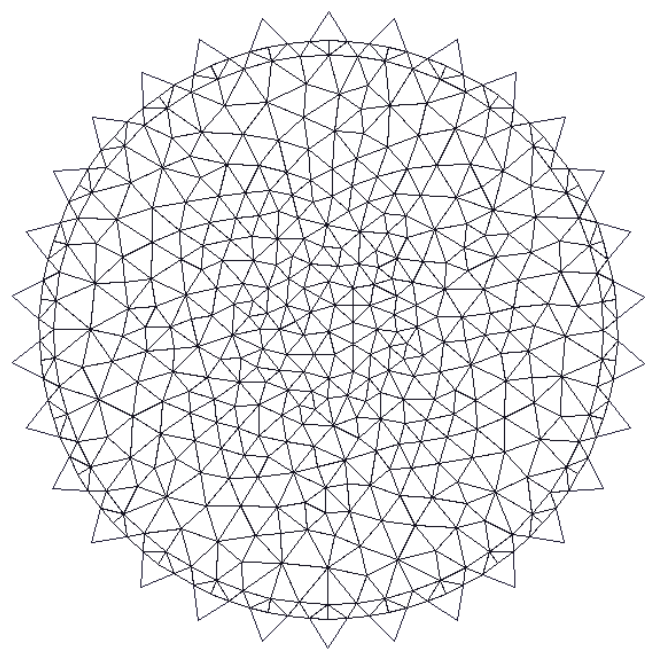

(b)

Figura 4.8: Malhas de elementos finitos para resolver o problema (a) direto e (b) inverso

\subsubsection{Dados de Simulação Numérica para o Método Gauss-Newton}

1. Objeto na borda: As simulações numéricas com objeto perto da borda vão ser feitas com malhas de elementos finitos em 2D. Foram usadas duas malhas diferentes para resolver o problema direto e inverso. A malha para o problema direto mostrada na figura 4.9(a) tem 2252 nós, 708 elementos e 32 eletrodos; enquanto que a malha para o problema inverso, tem 1604 nós, 492 elementos e 32 eletrodos como se observa na figura 4.9(b). Assumiu-se 
uma resistividade de $20 \Omega m$ para todos os elementos, exceto no objeto cuja resistividade adotou-se igual a $100 \Omega m$, o padrão de corrente e voltagem utilizado na medição do potencial elétrico foi pula três eletrodos.

2. Objeto no centro: A malha do problema direto mostrada na figura 4.8(a) tem 3522 nós, 1134 elementos e 32 eletrodos; enquanto que a malha do problema inverso mostrada na figura 4.8(b) tem 2508 nós, 796 elementos e 32 eletrodos. No problema direto admitiu-se uma resistividade homogênea de $20 \Omega m$ para todos os elementos, exceto para o objeto cuja resistividade adotou-se igual a $100 \Omega m$, o padrão de corrente e voltagem utilizado na medição do potencial elétrico foi pula três eletrodos.

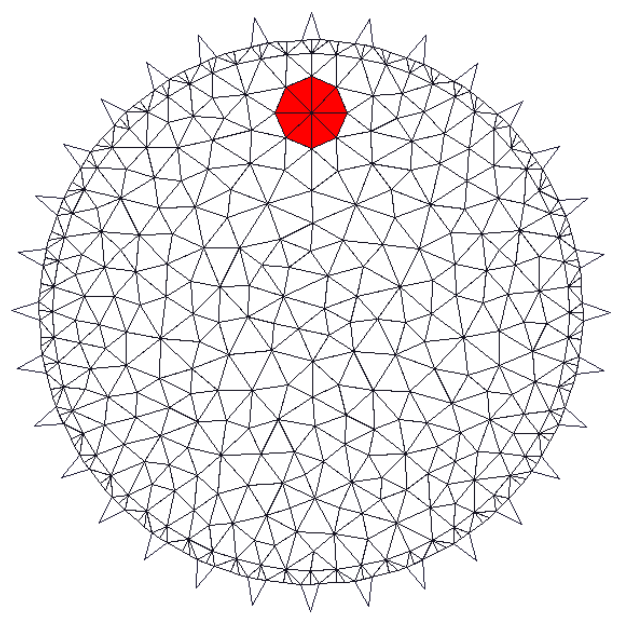

(a)

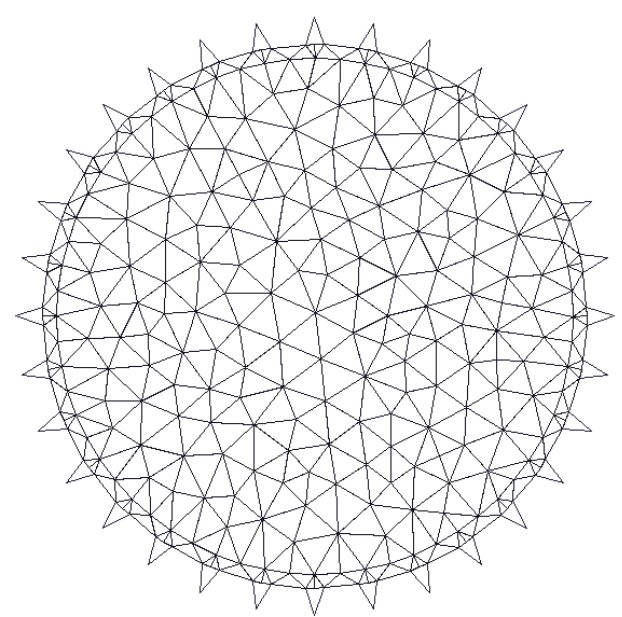

(b)

Figura 4.9: Malhas de elementos finitos em 2D para resolver o problema

(a) direto e (b) inverso

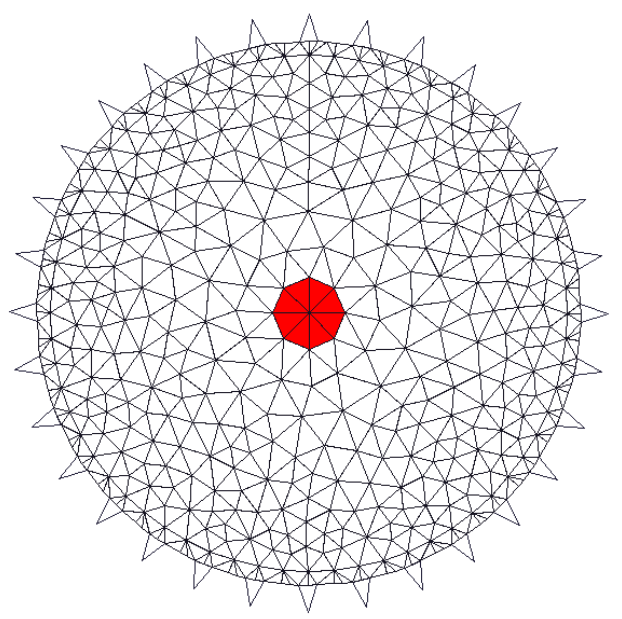

(a)

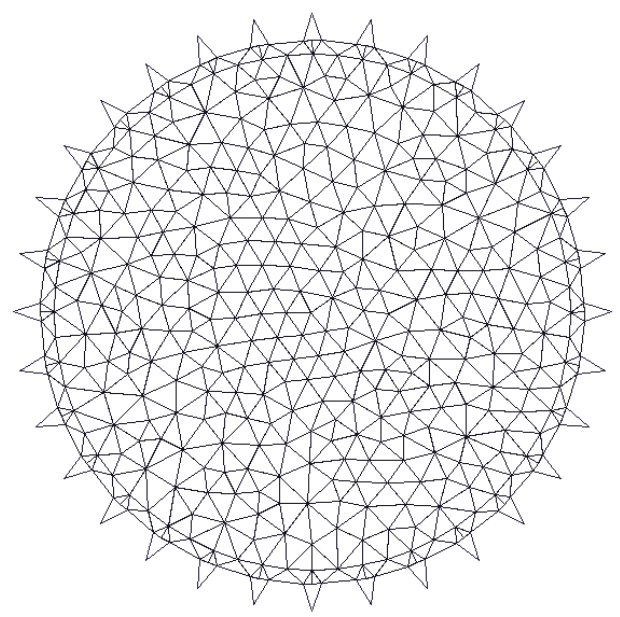

(b)

Figura 4.10: Malhas de elementos finitos em 2D para resolver o problema (a) direto e (b) inverso 


\subsubsection{Dados da Bancada Experimental}

\subsubsection{Dados de Bancada Experimental para Matriz de Sensibilidade}

Para trabalhar com dados da bancada experimental, foi gerada uma malha de 3306 nós, 1062 elementos e 30 eletrodos mostrada na figura 4.11. Assumiu-se parâmetros de eletrodo de $0,02 \Omega m^{2}$ e $20,0 \Omega m$ para a solução salina. O padrão de corrente e voltagem utilizado na medição do potencial elétrico foi pula um eletrodo.

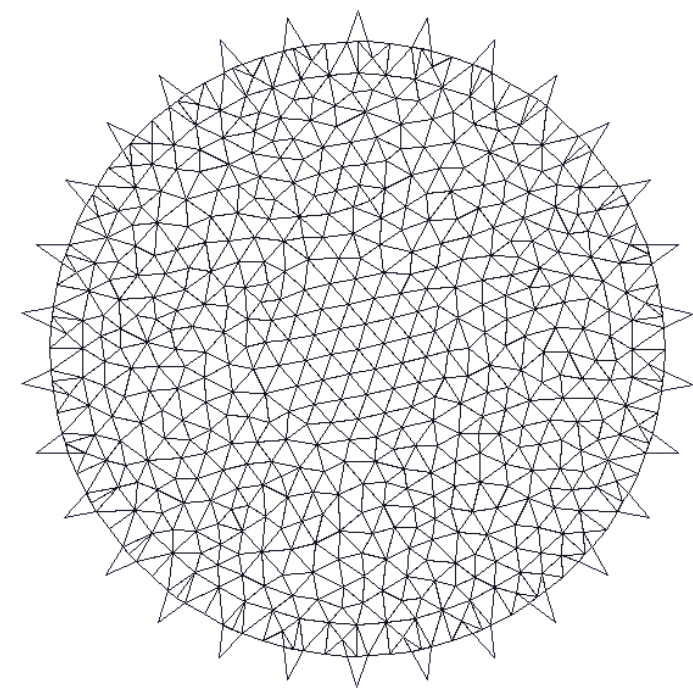

Figura 4.11: Malha de elementos finitos para trabalhar com dados da bancada experimental

\subsubsection{Dados de Bancada Experimental para Gauss-Newton}

Para trabalhar com dados da bancada experimental, foi gerada uma malha de 2516 nós, 796 elementos e 32 eletrodos mostrada na figura 4.12, está malha é utilizada para tratar dados com objeto e para tratar dados com agar. Com dados de fantoma com objeto, assumiu-se parâmetros do eletrodo de $0,02 \Omega \mathrm{m}^{2}$. A resistividade da solução salina foi medida, com o condutivímetro NT-CVP da Nova Técnica, com valor de $2,77 \Omega m$. A altura da solução salina é de $25 \mathrm{~mm}$ e o valor da corrente injetada foi de $4,0750 \mathrm{~mA}$ medida com um osciloscópio. O padrão de corrente e de voltagem utilizados na injeção de corrente e na medição do potencial elétrico foi pula três eletrodos.

Quando os objetos são de agar, os parâmetros de eletrodos foram adotados igual a $0,02 \Omega m$. A resistividade da solução salina foi medida, com o condutivíme-

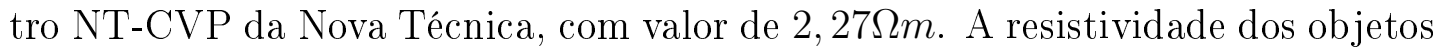
de agar simulando os pulmões foi medida, com o condutivímetro NT-CVP Nova 
Técnica, com valor de $3,8 \Omega \mathrm{m}$. A resistividade do objeto de agar para simular o coração foi determinada igual a $0,98 \Omega \mathrm{m}$. A altura da solução salina é de $20 \mathrm{~mm}$ e o valor da corrente injetada foi de 4,3249mA medida com osciloscópio. O padrão de corrente e o padrão de voltagem utilizado na medição do potencial elétrico foi pula três eletrodos.

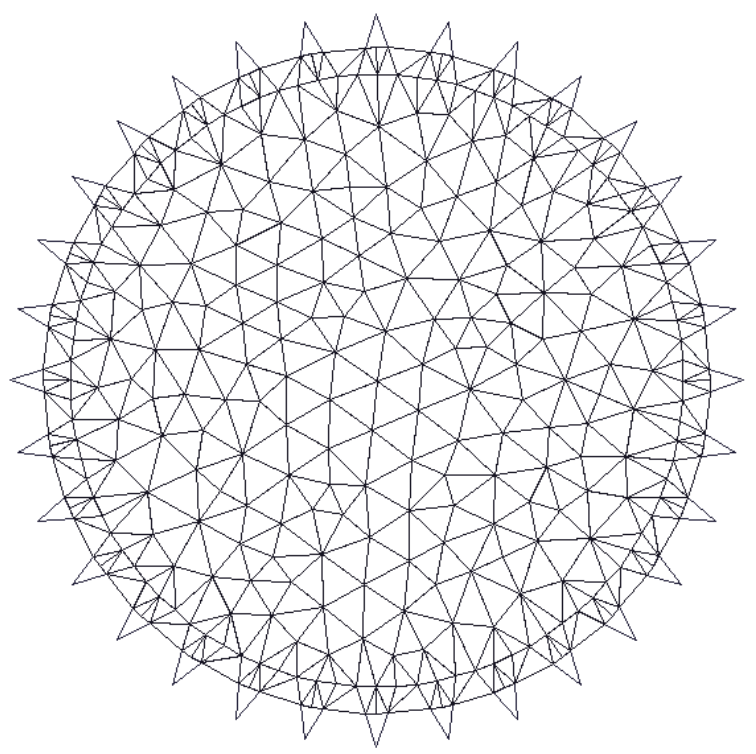

Figura 4.12: Malha de elementos finitos para trabalhar com dados da bancada experimental

\subsubsection{Dados do tórax humano para Gauss-Newton}

Para trabalhar com dados do tórax humano, os eletrodos foram alocados ao redor do tórax como mostrado nas figuras 4.4(a) e 4.4(b). Para gerar a malha de elementos finitos, duas medidas do corpo humano foram feitas como mostrado nas figuras $4.13(\mathrm{a})$ e $4.13(\mathrm{~b})$ 


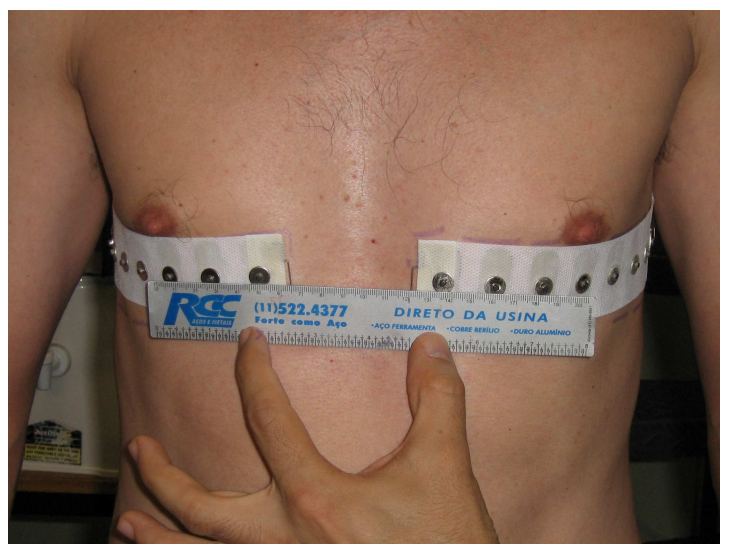

(a)

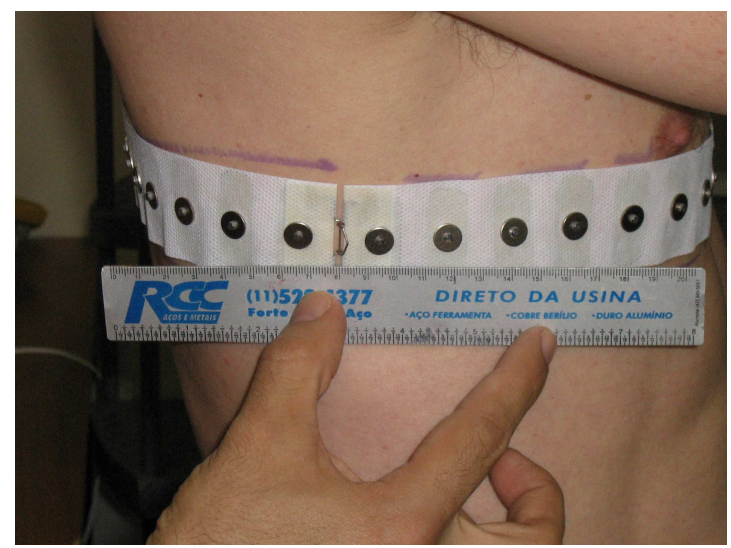

(b)

Figura 4.13: Medidas para gerar a malha de elementos finitos (a) distância entre eletrodos e (b) largura do tórax

A malha de elementos finitos gerada (figura 4.14) tem 1484 nós , 452 elementos e 32 eletrodos. Assumiu-se parâmetros dos eletrodos de $0,02 \Omega m^{2}$. O valor da corrente injetada foi de $2,725 \mathrm{~mA}$ medida com o osciloscopio. O padrão de corrente e o padrão de voltagem utilizado na medição do potencial elétrico foi pula três eletrodos. Os dados foram coletados quando o indivíduo estava em inspiração (pulmões com ar).

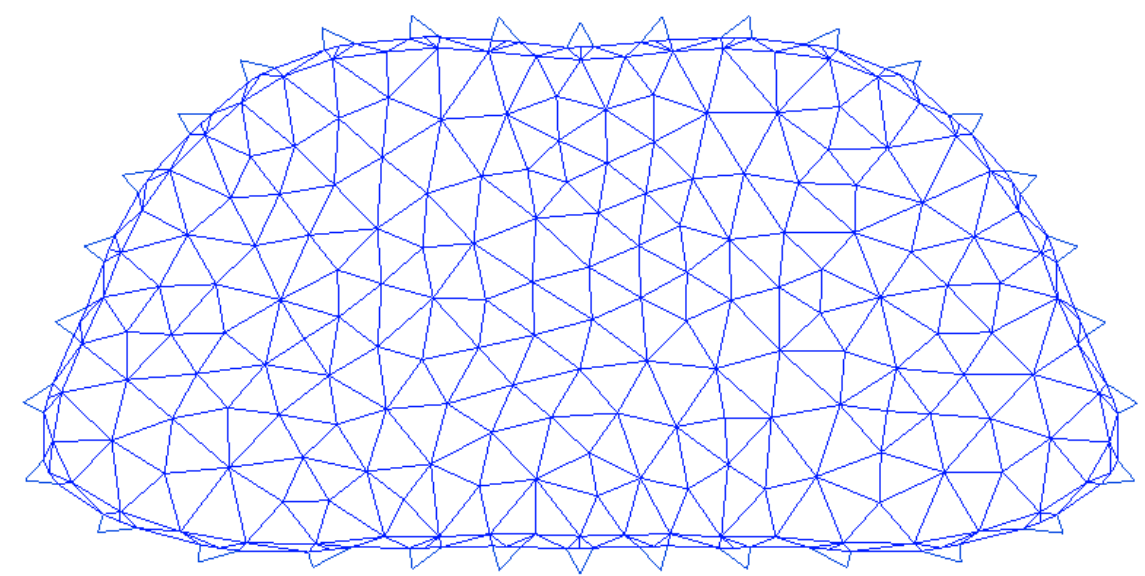

Figura 4.14: Malha de elementos finitos para gerar imagens do tórax humano

Os dados (objeto de acrílico, objetos de agar e tórax humano) foram coletados com o tomógrafo mostrado na figura 4.15 cedido pela empresa Dixtal. 


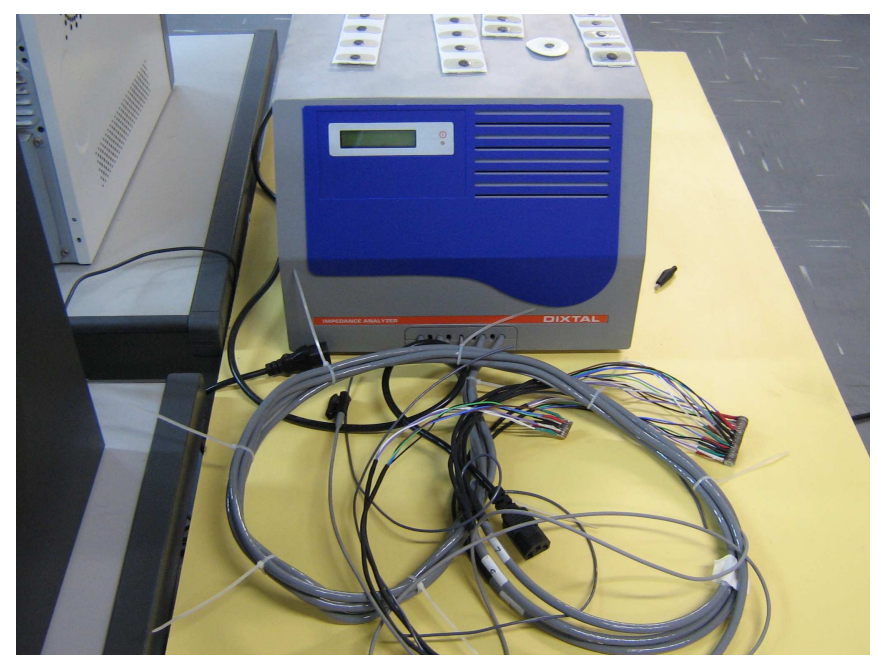

Figura 4.15: Tomógrafo por Impedância Elétrica 


\section{Resultados}

\subsection{Resultados Obtidos com PL na Diminuição do Erro Numérico}

Como anteriormente explicado, para investigar a diminuição do erro numérico trabalhando com PL calculou-se o índice $\left\|\mathbf{A A}^{-1}-\mathbf{I}=0\right\|$.

Nas figuras a continuação são apresentados gráficos dos autovalores da matriz A

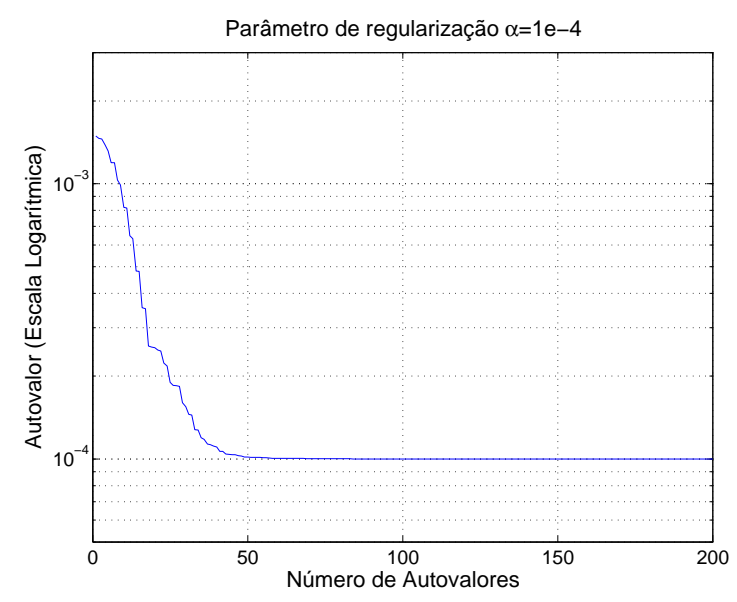

Figura 5.1: Autovalores para $\alpha=1,0 e^{-4}$ 


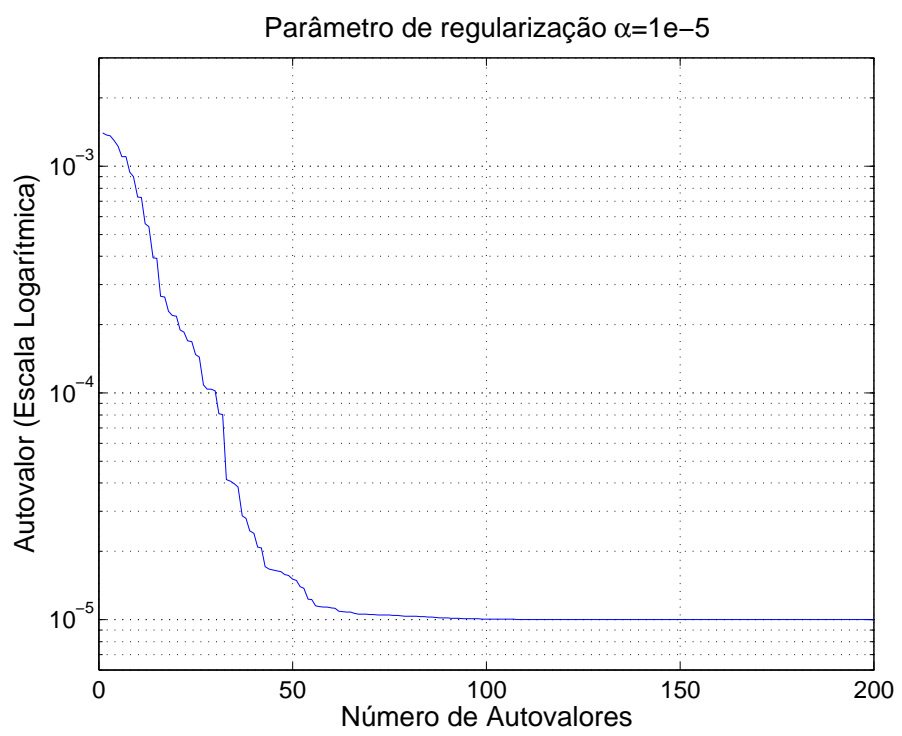

Figura 5.2: Autovalores para $\alpha=1,0 e^{-5}$

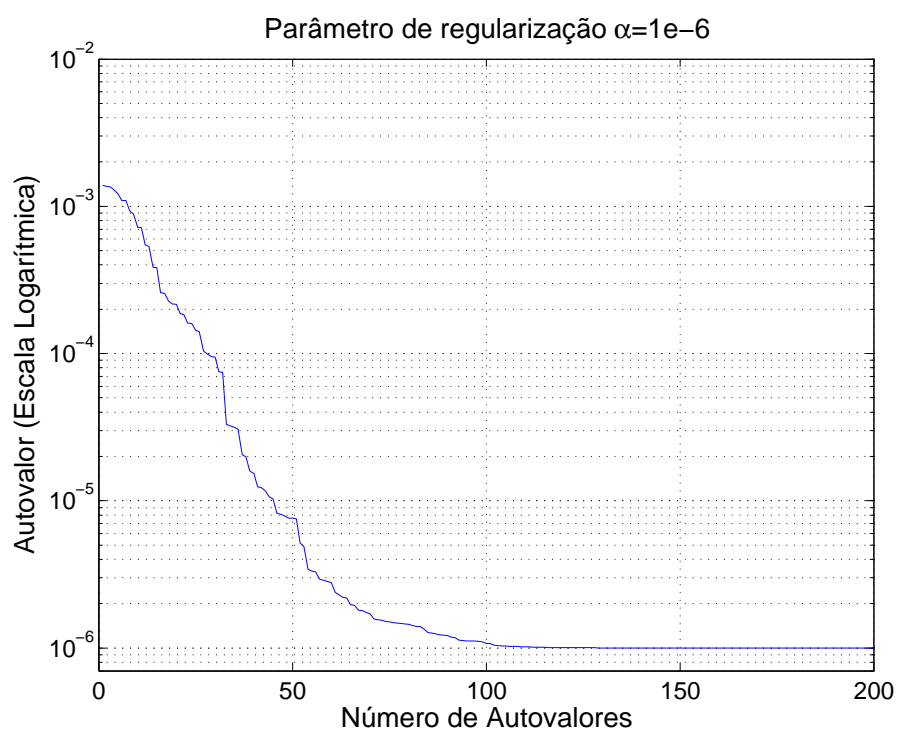

Figura 5.3: Autovalores para $\alpha=1,0 e^{-6}$ 


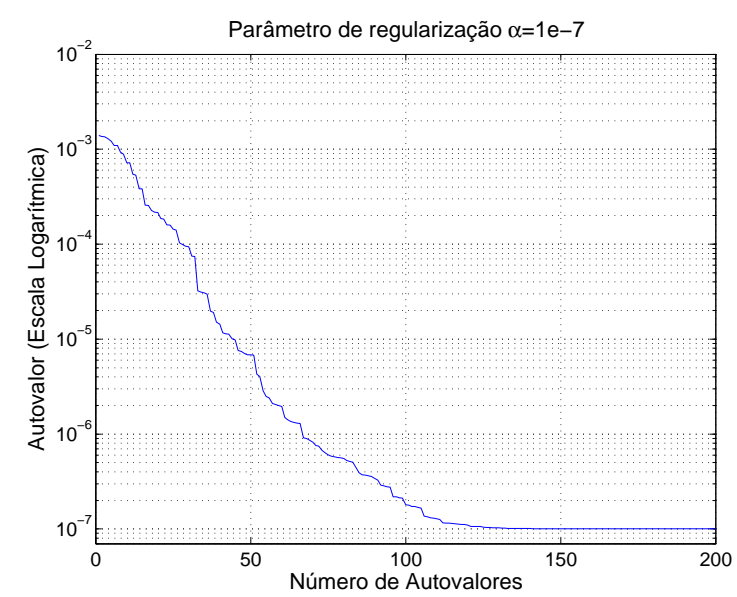

Figura 5.4: Autovalores para $\alpha=1,0 e^{-7}$

Nas figuras anteriormente mostradas, nota-se que o menor autovalor é igual ao valor da regularização e esses mesmos autovalores podem ser utilizados para definir um índice de observabilidade modal.

Nas tabelas apresentadas a seguir, mostram-se os resultados obtidos para diferentes valores de limite inferior e superior e para diferentes valores de parâmetros de regularização $\alpha$. Para poder fazer uma comparação direta entre PL e LU Decomposition, é necessário encontrar uma solução para o sistema linear com PL onde o número de restrições ativas $\mathbf{R a}$ seja igual a zero.

\begin{tabular}{|c|c|c|c|c|c|}
\hline Limite Inferior & Limite Superior & Parâmetro $\alpha$ & Erro PL & Erro LU & Ra \\
\hline 0.8 & 1.2 & $1,0 e^{-4}$ & $2,4940 e^{-12}$ & $2,3261,0 e^{-12}$ & 12 \\
\hline 0.8 & 1.2 & $1,0 e^{-5}$ & $2,0137 e^{-11}$ & $1,8730 e^{-11}$ & 2 \\
\hline 0.8 & 1.2 & $1,0 e^{-6}$ & $1,5893 e^{-10}$ & $3,3242 e^{-10}$ & 0 \\
\hline 0,8 & 1.2 & $1,0 e^{-7}$ & $1,2087 e^{-9}$ & $6,7121,0 e^{-9}$ & 0 \\
\hline
\end{tabular}

Tabela 5.1: Os limites são determinados somando e subtraindo $20 \%$ respectivamente 


\begin{tabular}{|c|c|c|c|c|c|}
\hline Limite Inferior & Limite Superior & Parâmetro $\alpha$ & Erro PL & Erro LU & Ra \\
\hline 0.7 & 1.3 & $1,0 e^{-4}$ & $2,4940 e^{-12}$ & $2,3261,0 e^{-12}$ & 8 \\
\hline 0.7 & 1.3 & $1,0 e^{-5}$ & $2,0137 e^{-11}$ & $1,8730 e^{-11}$ & 2 \\
\hline 0.7 & 1.3 & $1,0 e^{-6}$ & $1,5893 e^{-10}$ & $3,3242 e^{-10}$ & 0 \\
\hline 0,7 & 1.3 & $1,0 e^{-7}$ & $1,2087 e^{-9}$ & $6,7121,0 e^{-9}$ & 0 \\
\hline
\end{tabular}

Tabela 5.2: Os limites são determinados somando e subtraindo $30 \%$ respectivamente

\subsection{Resultados Implementando PL e LU Decom- position no Problema de Imagens de Variação de Resistividade}

\subsubsection{Dados de Simulação Numérica}

Na solução do problema inverso, para o primeiro e segundo testes, utilizou-se uma estimativa inicial de resistividade homogênea de $20 \Omega m$ e $0,02 \Omega m^{2}$ para os parâmetros dos eletrodos. Para o terceiro e quarto teste, a estimativa inicial de resistividade foi de $10 \Omega m$ e $0,02 \Omega m^{2}$ para os parâmetros dos eletrodos. Foram usadas as malhas das figuras 4.6(a) e 4.6(b) para o primeiro e segundo teste. Para o terceiro teste foram usadas as malhas das figuras 4.7(a) e 4.7(b). No último teste as malhas usadas são mostradas nas figuras 4.8(a) e 4.8(b). A seguir são mostradas as imagens ${ }^{1}$ geradas em cada teste

- Elemento perturbado perto da borda Parâmetro $\alpha=1,0 e^{-2}$

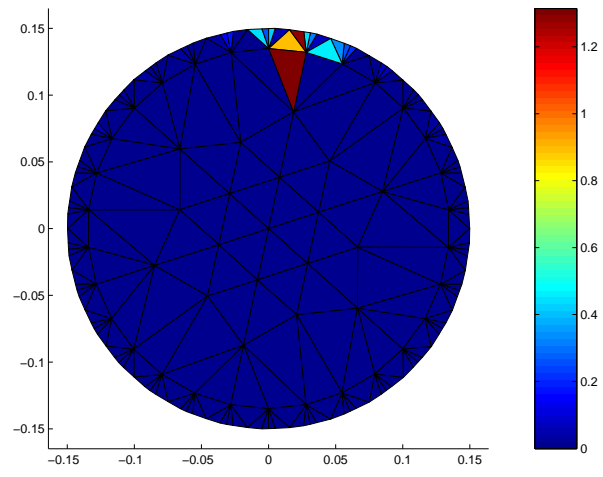

(a)

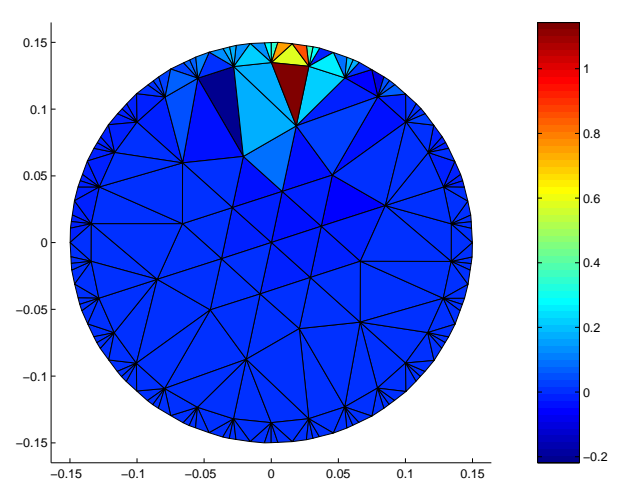

(b)

Figura 5.5: Distribuição de resistividade, elemento perturbado na borda (a) com PL e (b) LU Decomposition

\footnotetext{
${ }^{1}$ As imagens foram feitas no software "Matlab"e no "Gnuplot"onde o domínio circular está inscrito numa forma retangular.
} 


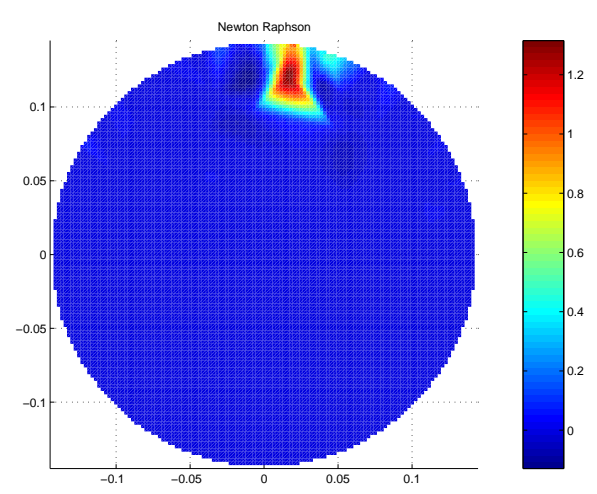

(a)

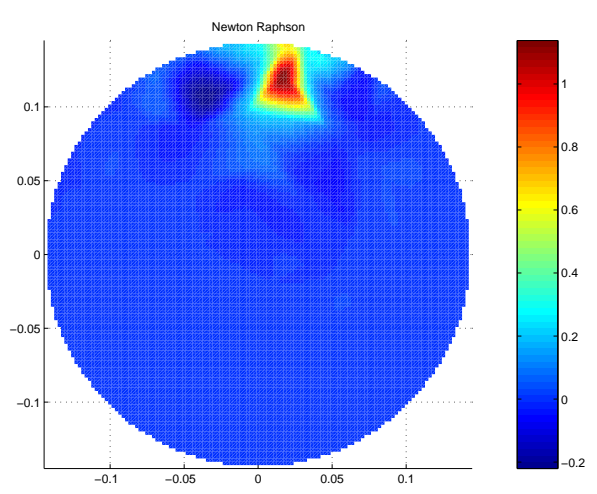

(b)

Figura 5.6: Imagem interpolada (a) com PL e (b) LU Decomposition

- Elemento perturbado perto do centro Parâmetro $\alpha=1,0 e^{-2}$

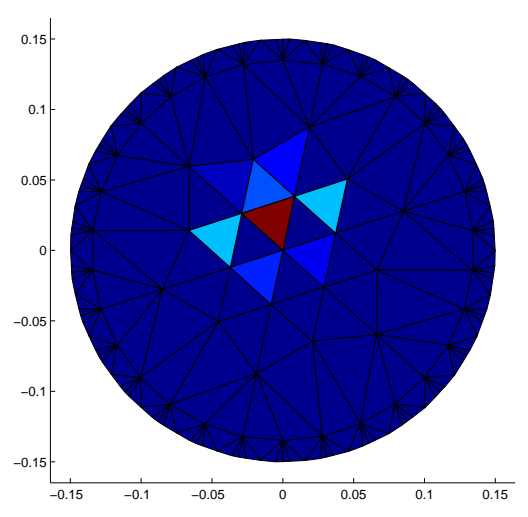

(a)
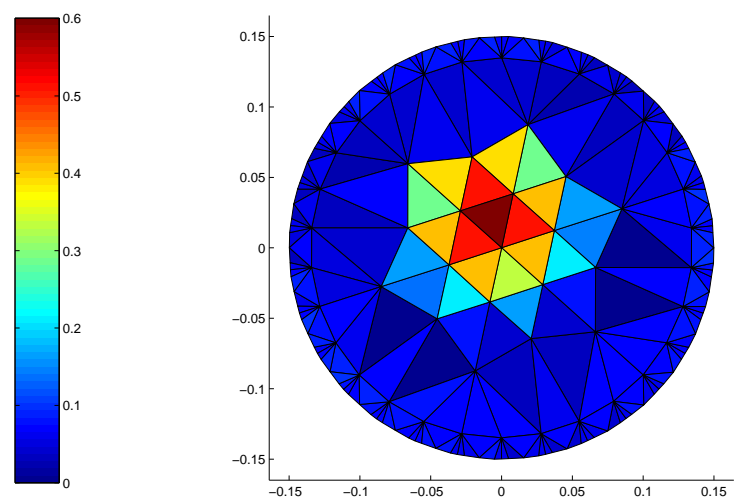

(b)

Figura 5.7: Distribuição de resistividade, elemento perturbado no centro (a) com PL e (b) LU Decomposition

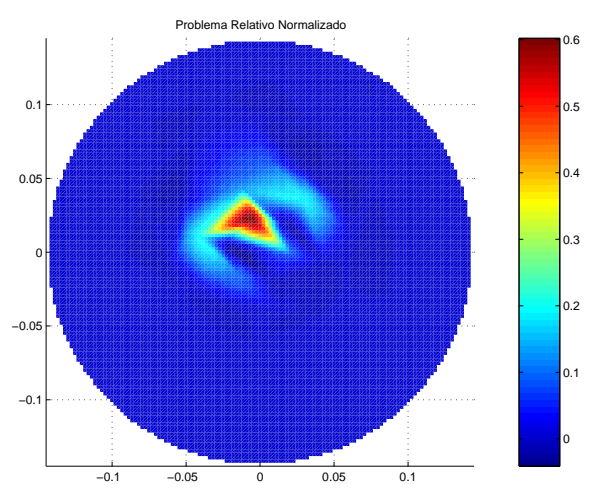

(a)

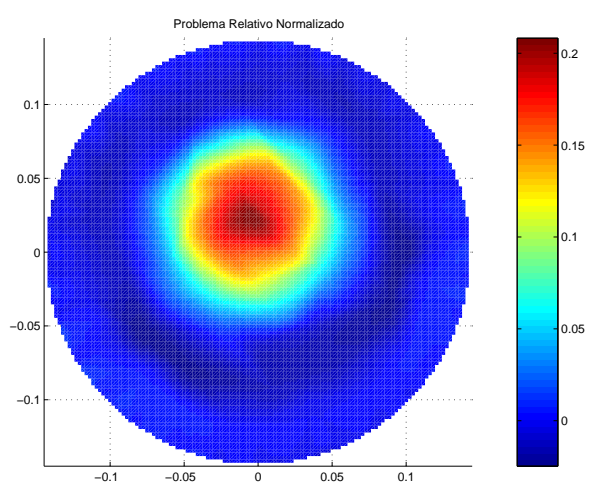

(b)

Figura 5.8: Imagem interpolada (a) com PL e (b) LU Decomposition 
- Objeto perturbado perto da borda Parâmetro $\alpha=1,0 e^{-2}$

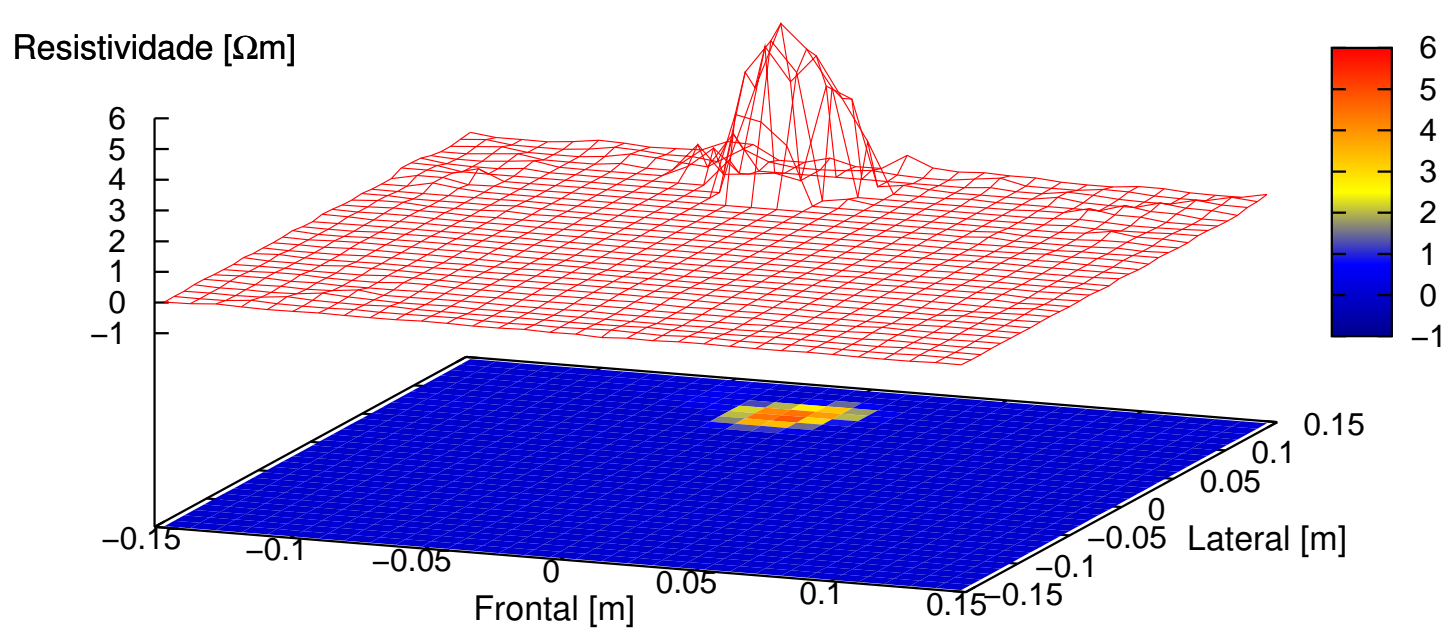

Figura 5.9: Distribuição de resistividade, imagem interpolada, objeto na borda com dados experimentais utilizando PL

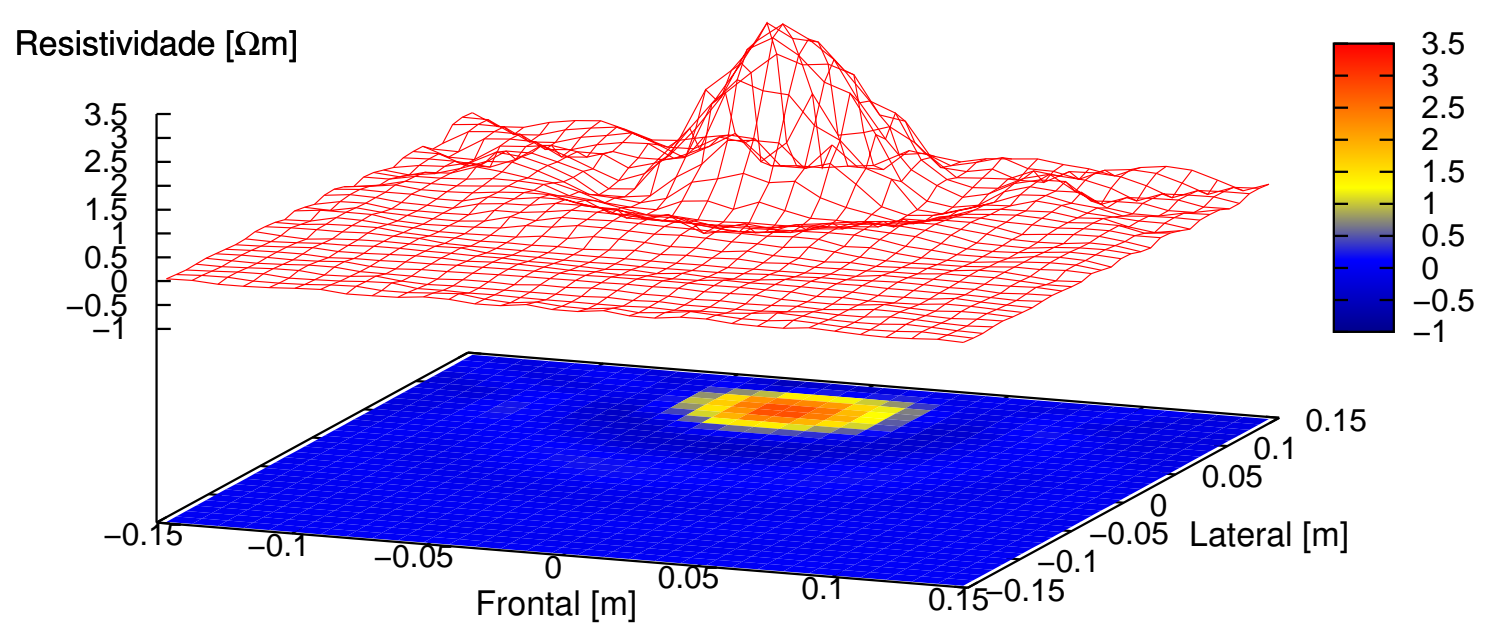

Figura 5.10: Distribuição de resistividade, imagem interpolada, objeto na borda com dados experimentais utilizando LU Decomposition 
- Objeto perturbado perto da borda Parâmetro $\alpha=0,0$

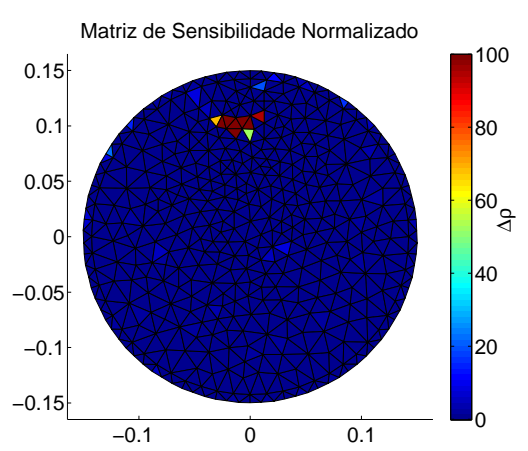

(a)

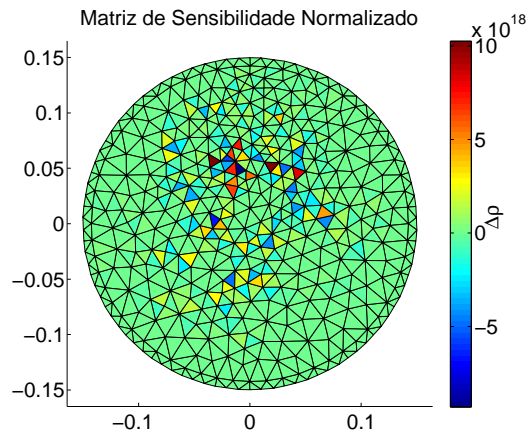

(b)

Figura 5.11: Distribuição de resistividade, objeto na borda com dados experimentais (a) com PL e (b) LU Decomposition

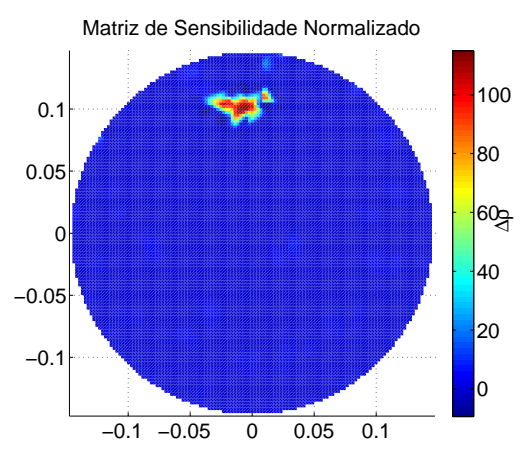

(a)

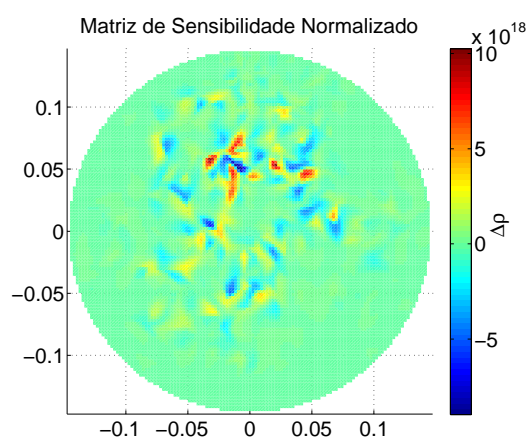

(b)

Figura 5.12: Imagem interpolada (a) com PL e (b) LU Decomposition

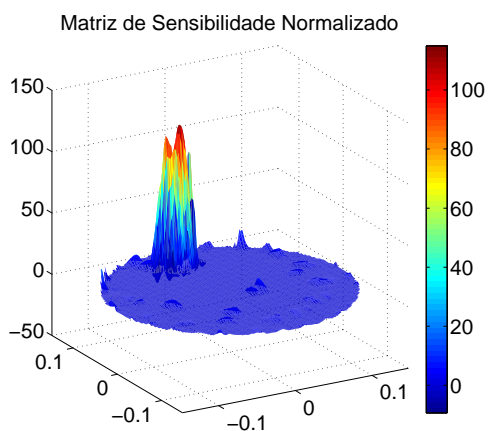

(a)

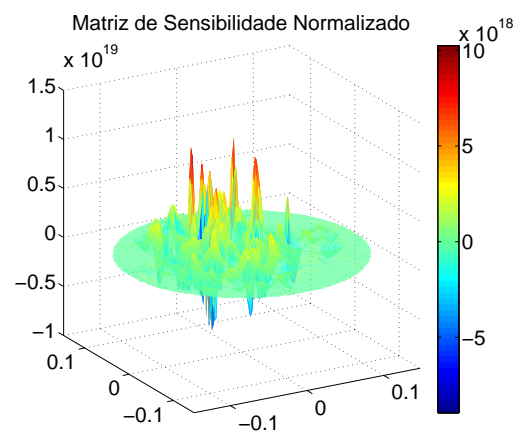

(b)

Figura 5.13: Imagem interpolada em perspectiva (a) com PL e (b) LU Decomposition 
- Objeto perturbado perto do centro Parâmetro $\alpha=1,0 e^{-2}$

Resistividade $[\Omega \mathrm{m}]$

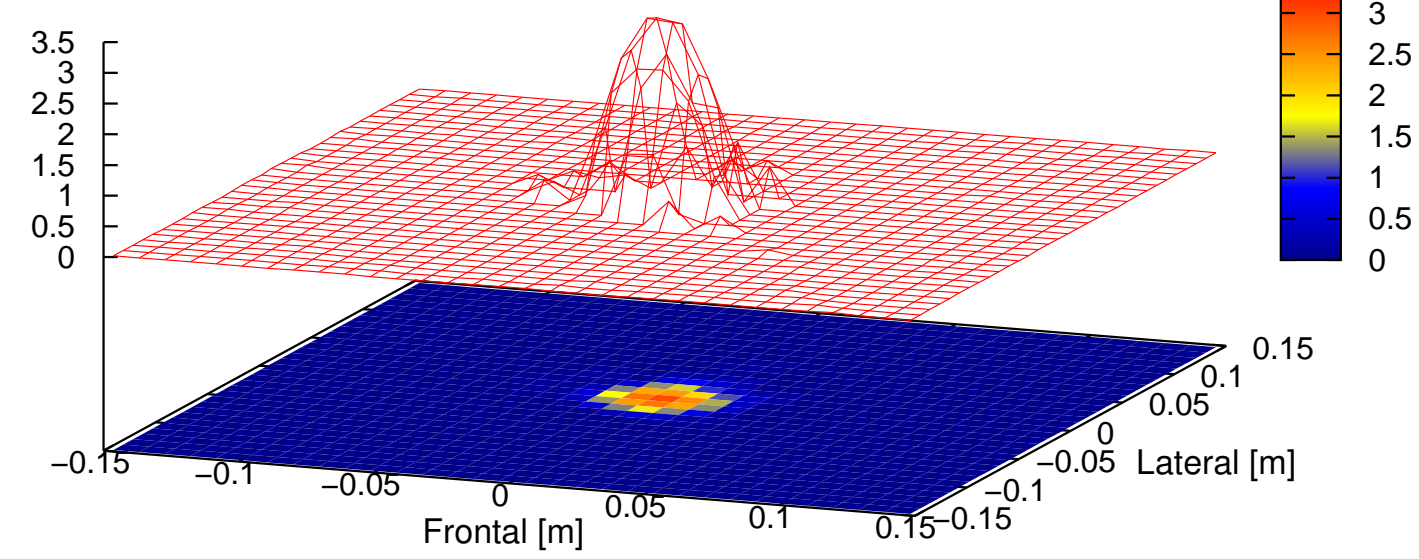

Figura 5.14: Distribuição de resistividade, objeto no centro com dados experimentais, solução via $\mathrm{PL}$

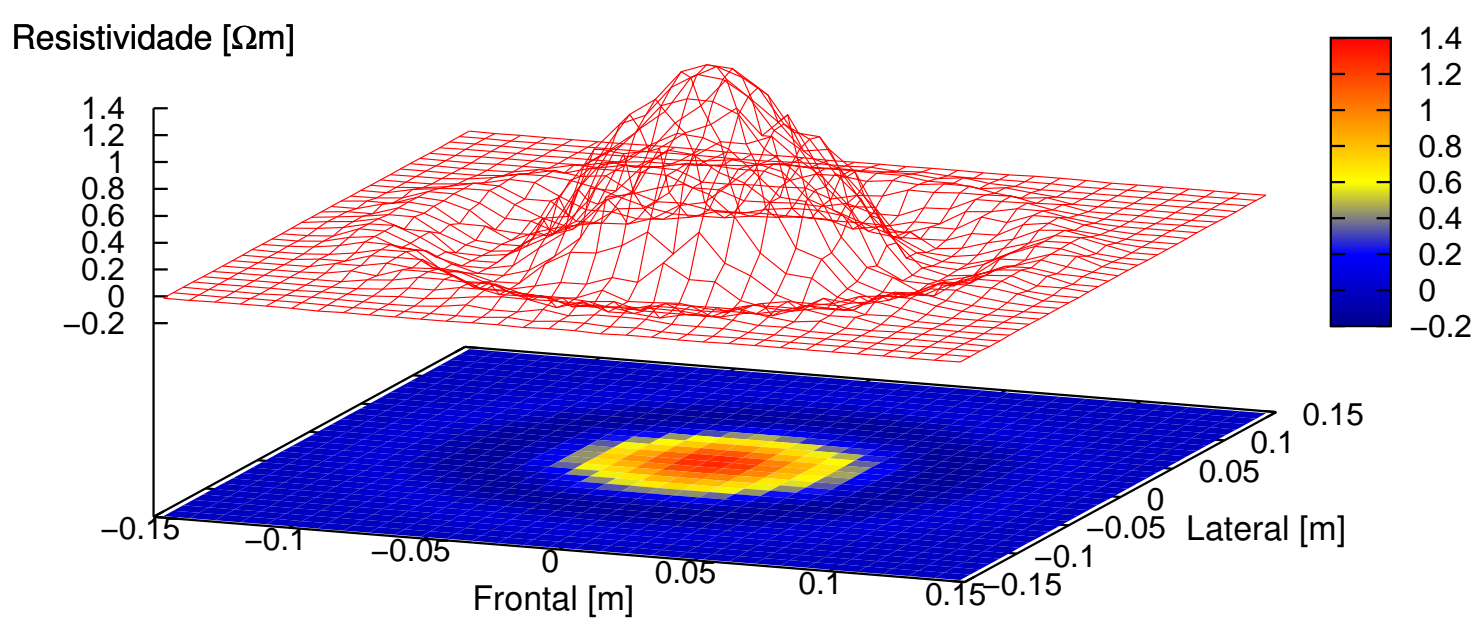

Figura 5.15: Distribuição de resistividade, imagem interpolada, objeto no centro com dados experimentais, solução via LU Decomposition 
- Objeto perturbado perto do centro Parâmetro $\boldsymbol{\alpha}=\mathbf{0 , 0}$

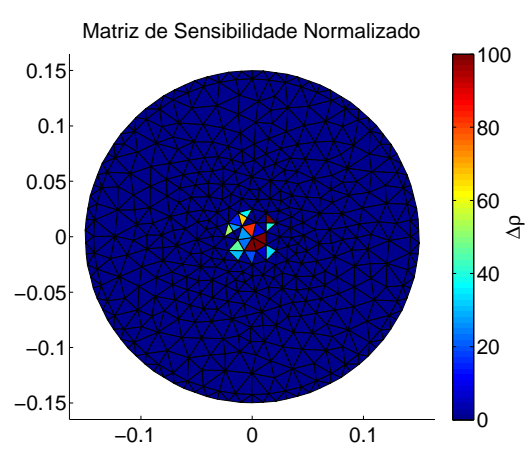

(a)

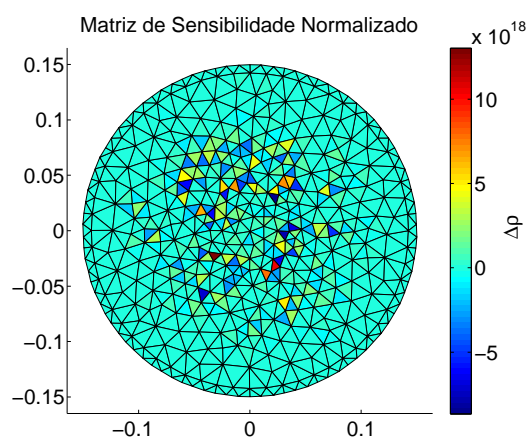

(b)

Figura 5.16: Distribuição de resistividade no centro com dados experimentais (a) com PL e (b) LU Decomposition

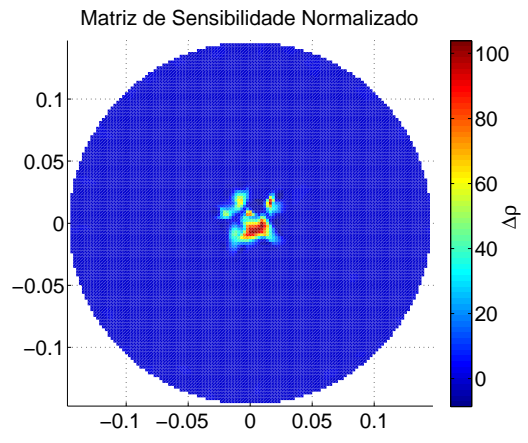

(a)

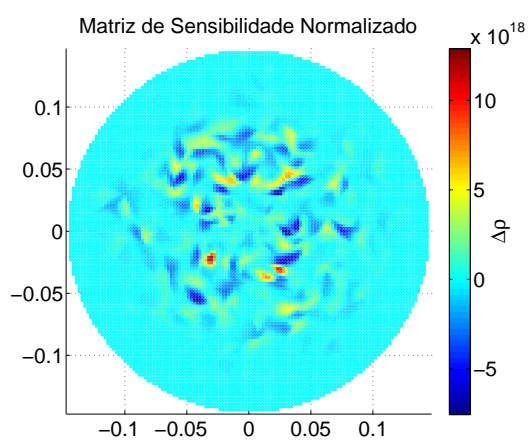

(b)

Figura 5.17: Imagem interpolada (a) com PL e (b) LU Decomposition

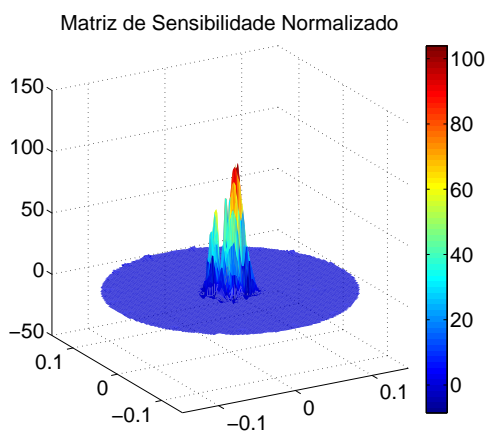

(a)

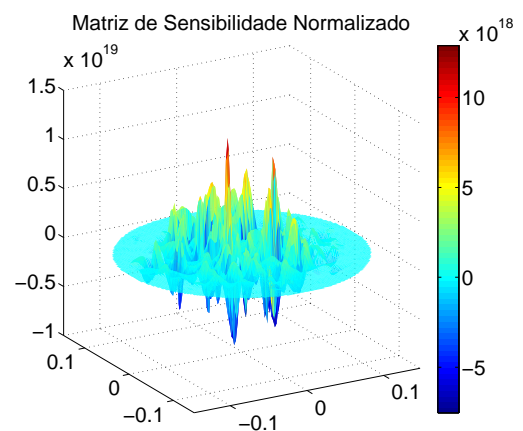

(b)

Figura 5.18: Imagem interpolada em perspectiva (a) com PL e (b) LU Decomposition 


\subsubsection{Dados da Bancada Experimental}

Para a obtenção de dados da bancada experimental o objeto foi posicionado na borda e no centro, conforme mostrado nas figuras 4.2(a) e 4.2(b). Foi imposto um valor de $0,02 \Omega m^{2}$ para o parâmetro do eletrodo e $20,0 \Omega m$ para a solução salina. Para cada posição do objeto, vai ser gerada imagem ${ }^{2}$ com diferentes valores de $\alpha$, permitindo observar o comportamento de PL quando a matriz vai-se tornando mal condicionada.

- Objeto perto da borda Parâmetro $\alpha=1,0 e^{-3}$

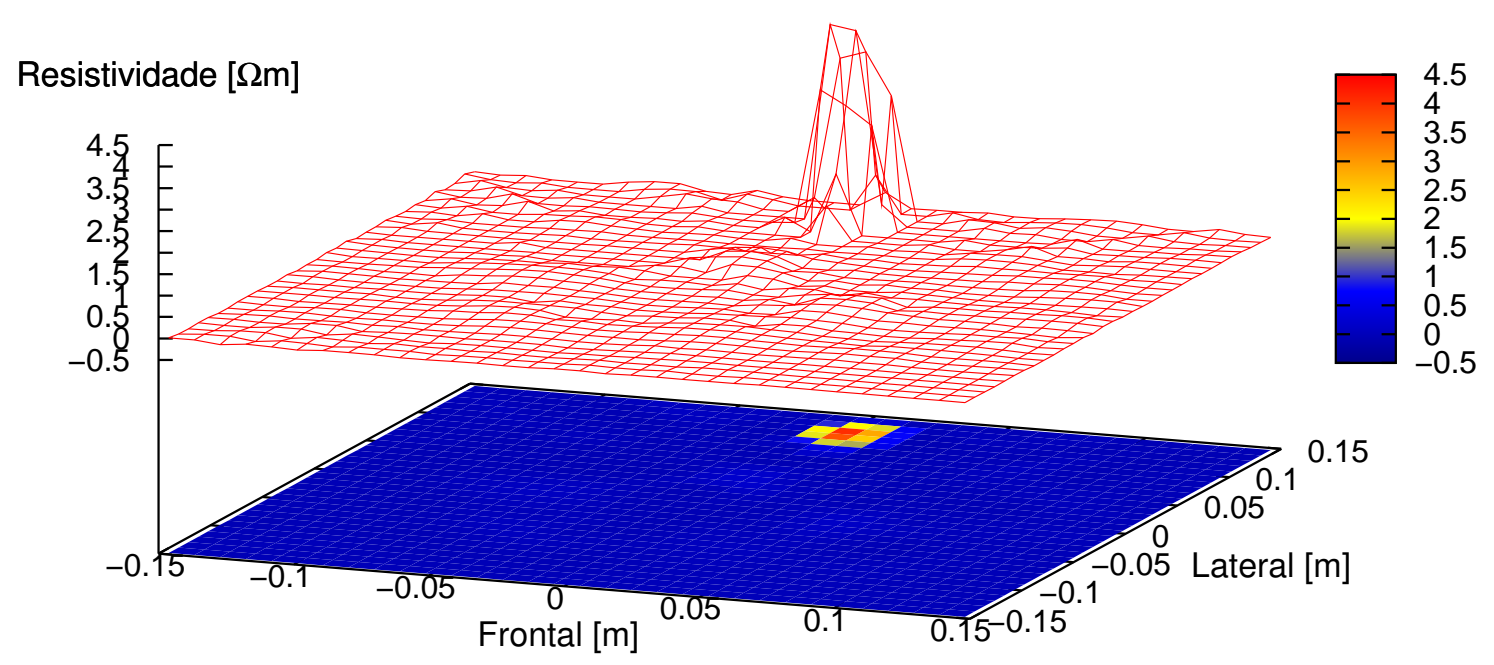

Figura 5.19: Obtenção de imagem de variação de resistividade na borda com dados experimentais utilizando PL

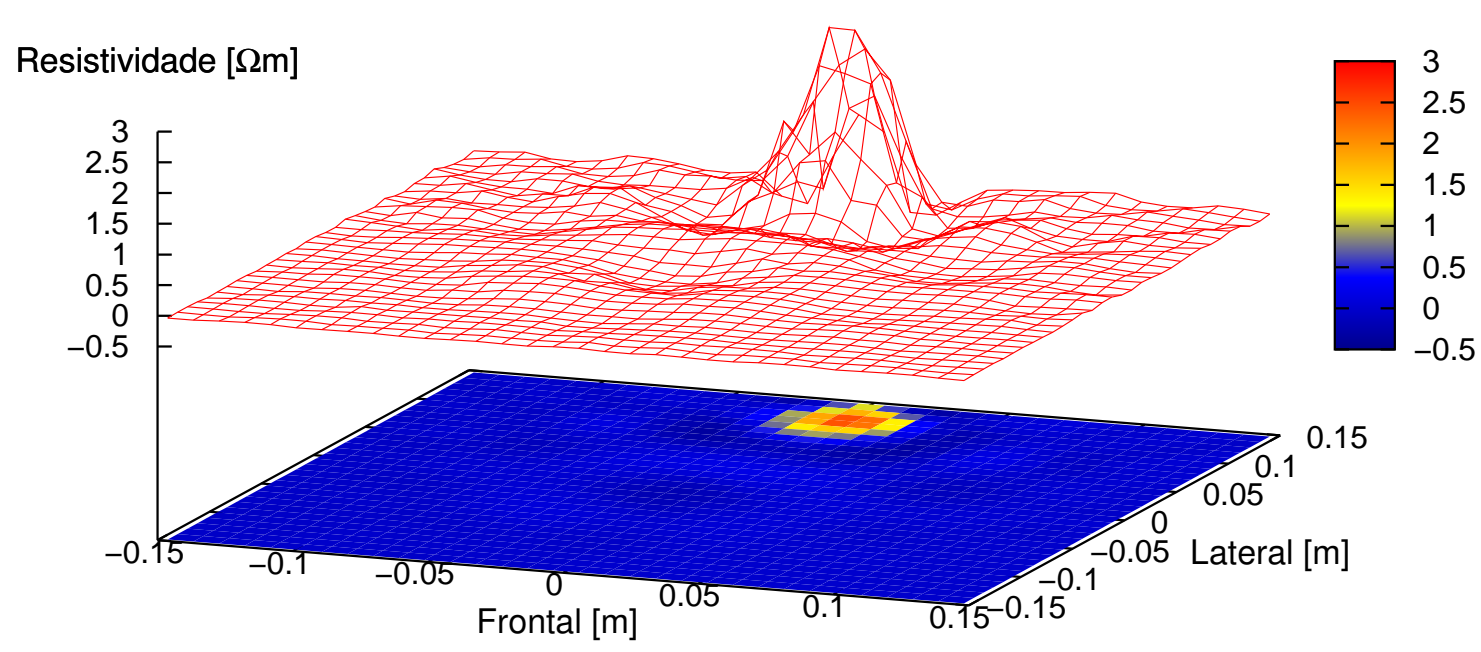

Figura 5.20: Obtenção de imagem de variação de resistividade na borda com dados experimentais utilizando LU Decomposition

\footnotetext{
${ }^{2}$ As imagens foram feitas no software "Matlab"e no "Gnuplot"onde o domínio circular está inscrito numa forma retangular.
} 
- Objeto perto da borda Parâmetro $\alpha=1,0 e^{-4}$

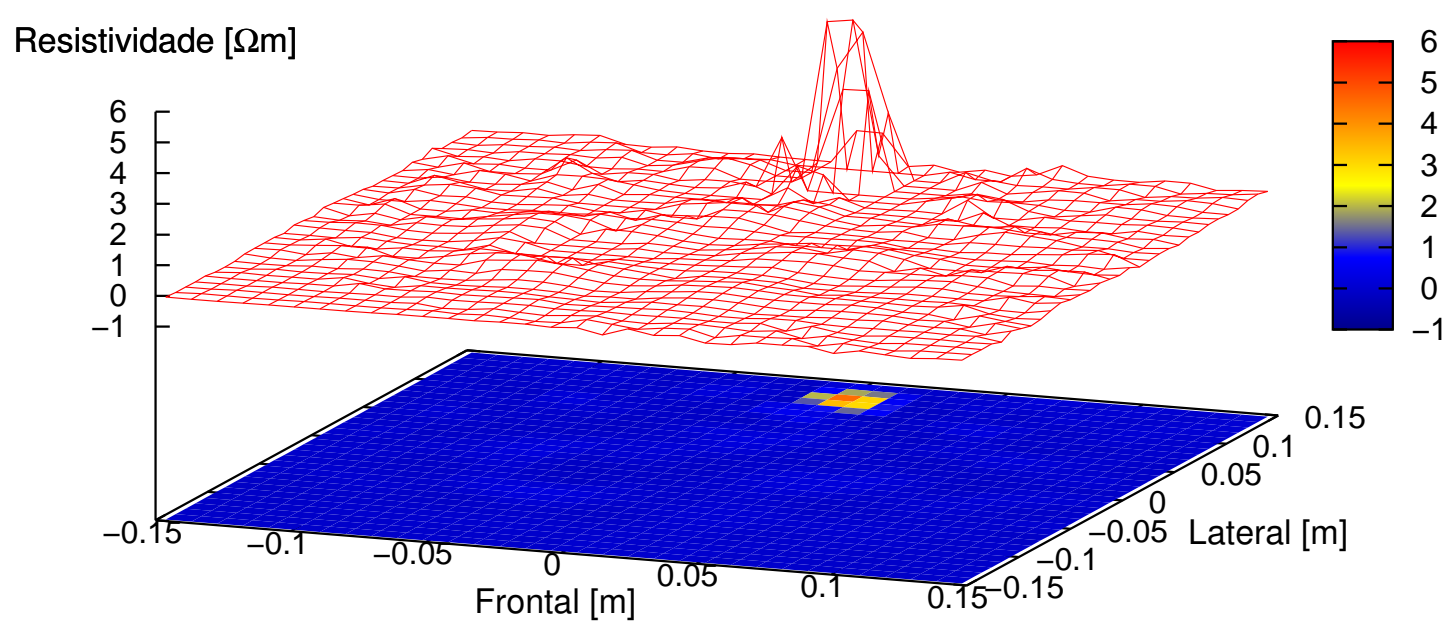

Figura 5.21: Obtenção de imagem de variação de resistividade na borda com dados experimentais utilizando PL

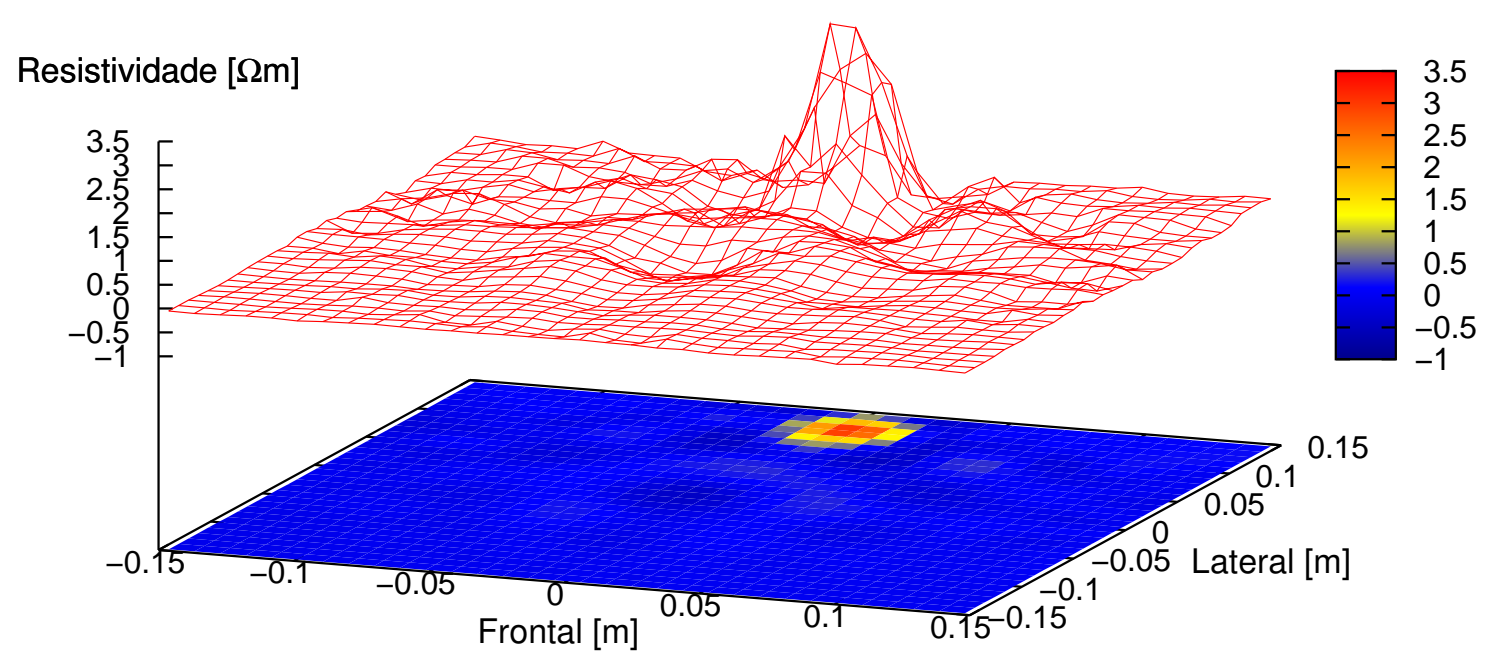

Figura 5.22: Obtenção de imagem de variação de resistividade na borda com dados experimentais utilizando LU Decomposition 
- Objeto perto da borda Parâmetro $\alpha=0,0$

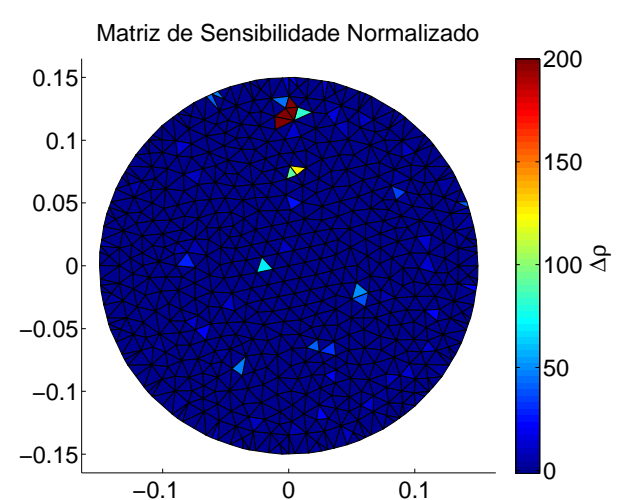

(a)

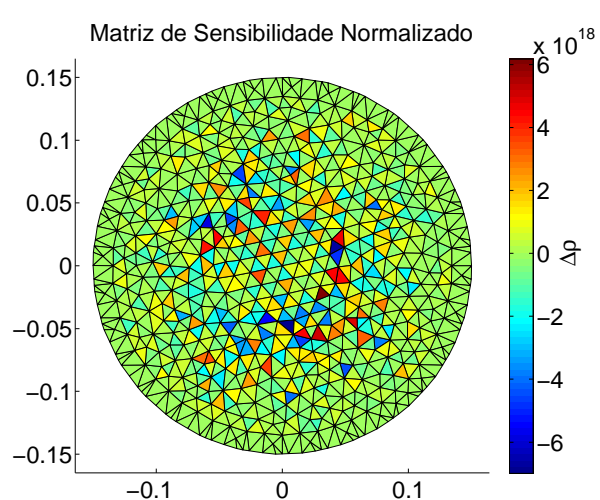

(b)

Figura 5.23: Obtenção da distribuição de resistividade na borda com dados experimentais (a) com PL e (b) LU Decomposition

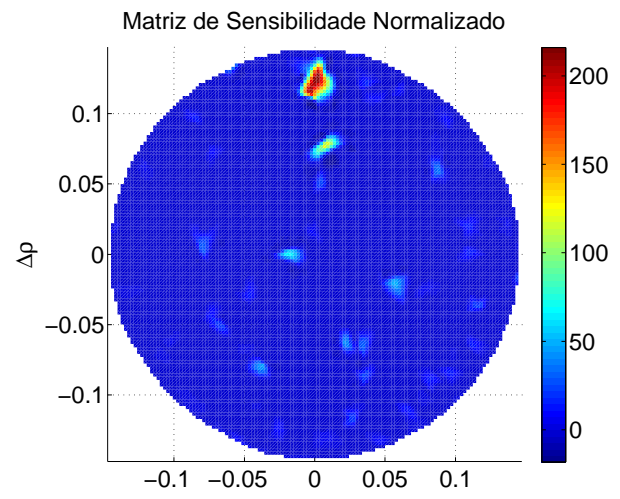

(a)

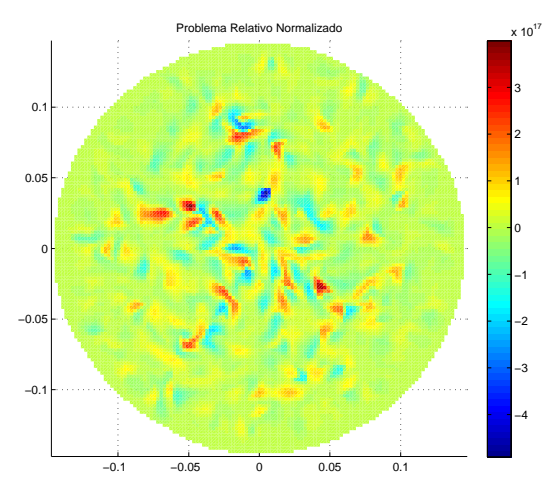

(b)

Figura 5.24: Imagem interpolada (a) com PL e (b) LU Decomposition

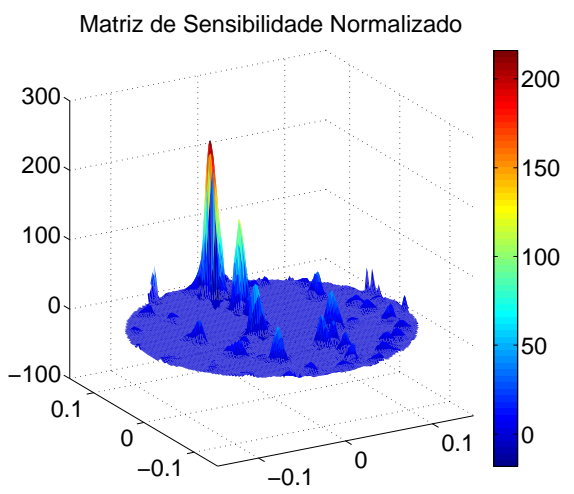

(a)

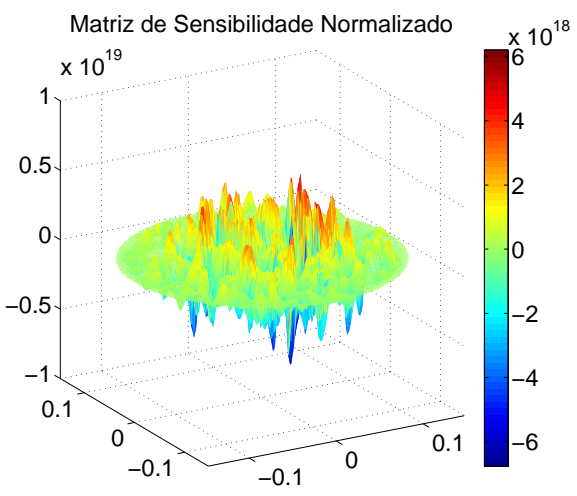

(b)

Figura 5.25: Imagem interpolada em perspectiva (a) com PL e (b) LU 
- Objeto perto do centro Parâmetro $\alpha=1,0 e^{-3}$

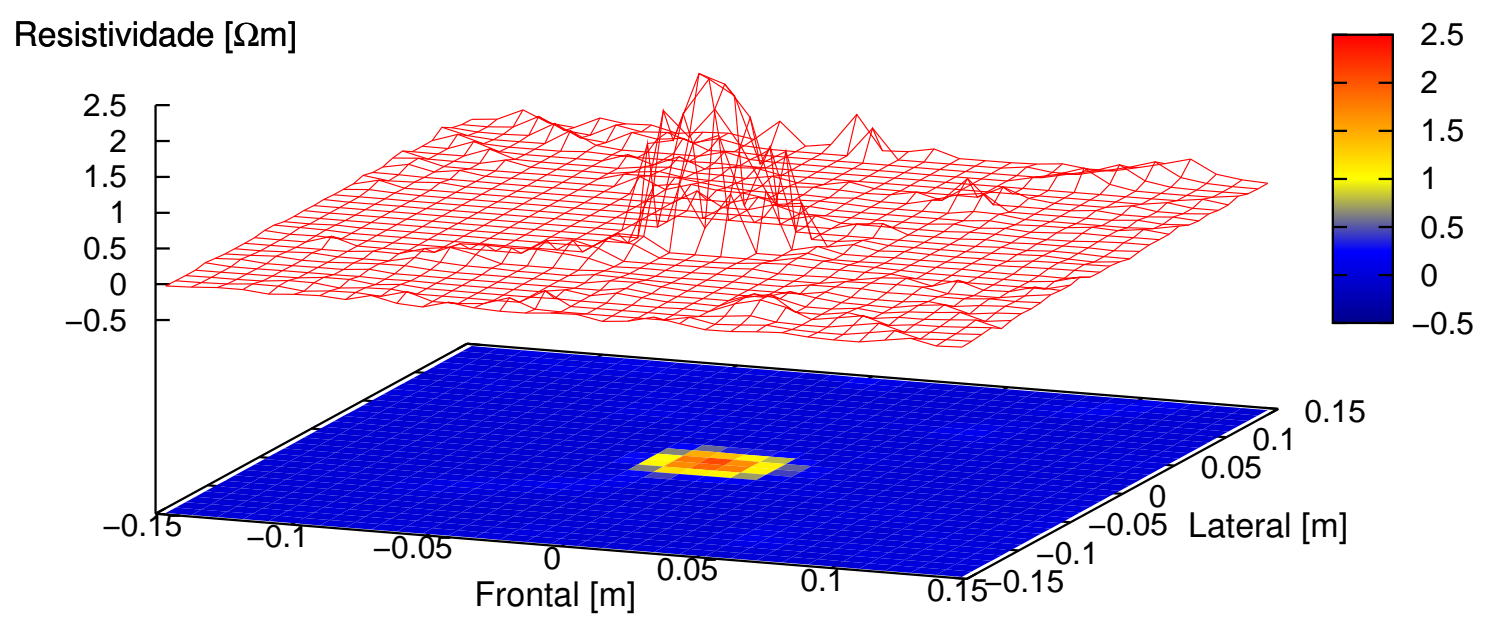

Figura 5.26: Obtenção de imagem de variação de resistividade na borda com dados experimentais utilizando PL

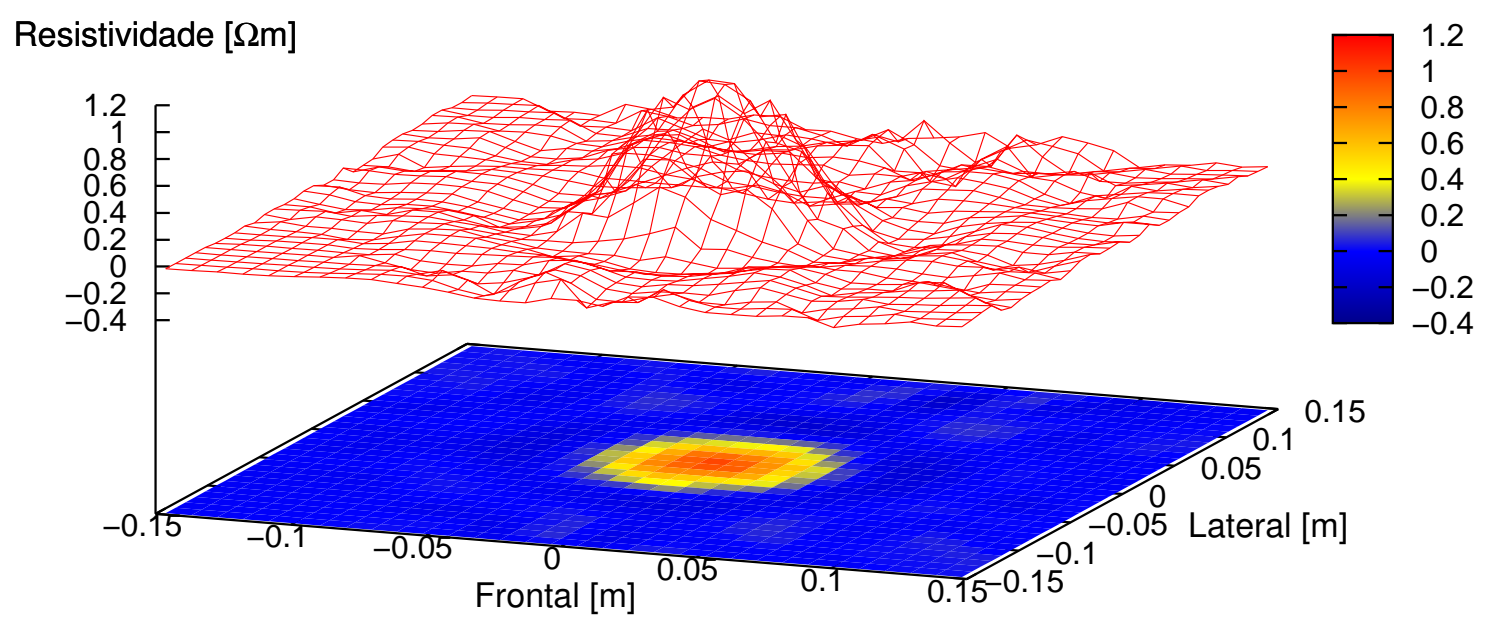

Figura 5.27: Obtenção de imagem de variação de resistividade na borda com dados experimentais utilizando LU Decomposition 
- Objeto perto do centro Parâmetro $\alpha=1,0 e^{-4}$

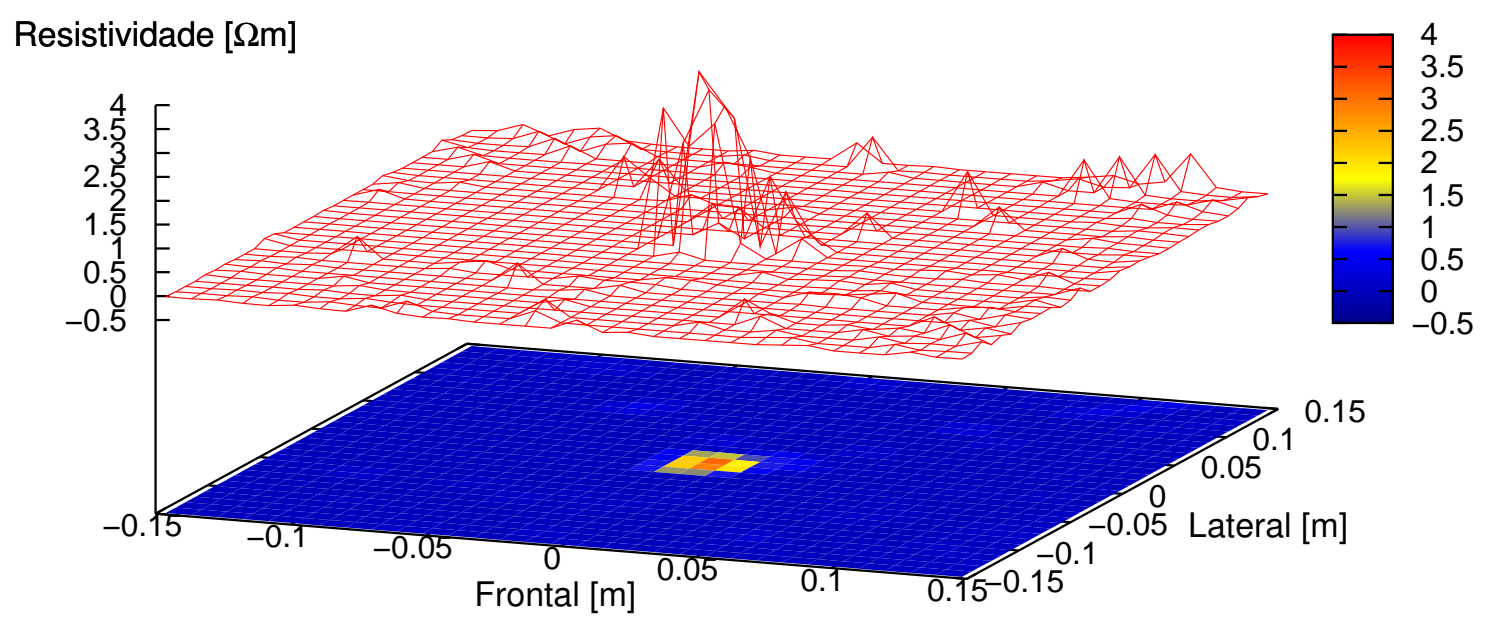

Figura 5.28: Obtenção de imagem de variação de resistividade na borda com dados experimentais utilizando PL

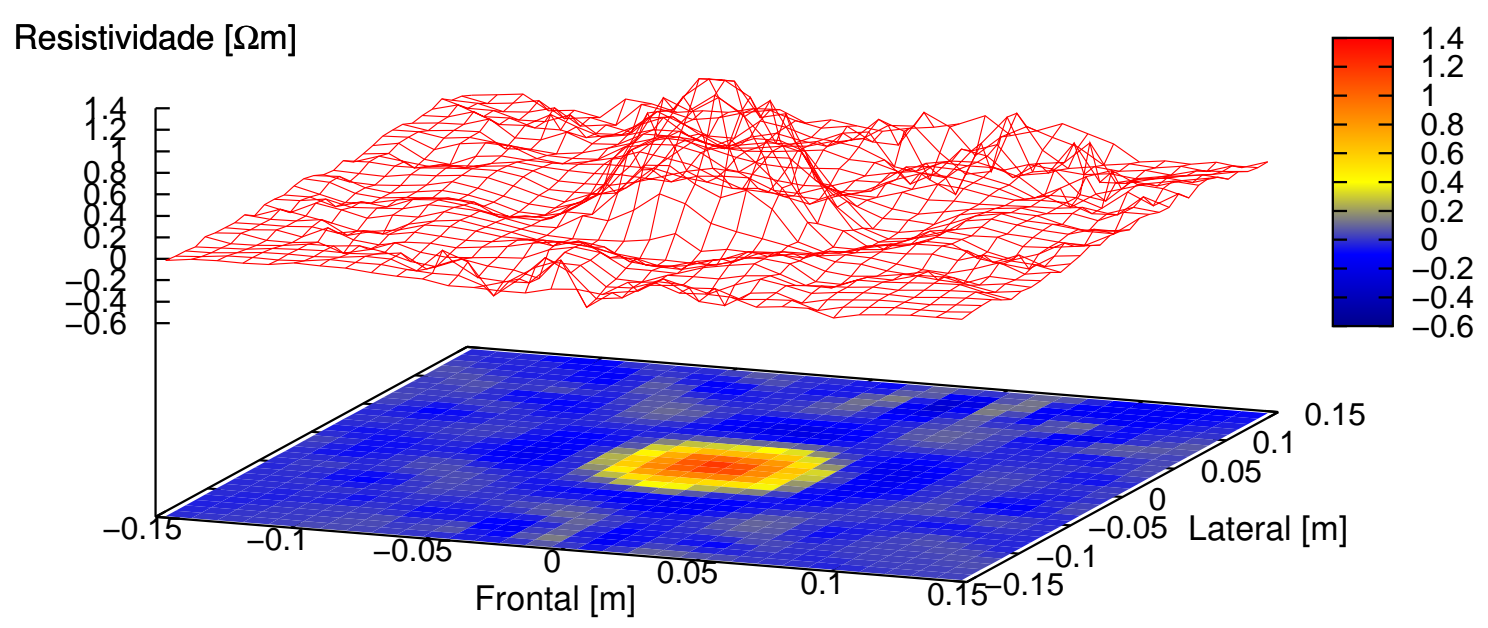

Figura 5.29: Obtenção de imagem de variação de resistividade na borda com dados experimentais utilizando LU Decomposition 
- Objeto perto do centro Parâmetro $\boldsymbol{\alpha}=\mathbf{0 , 0}$

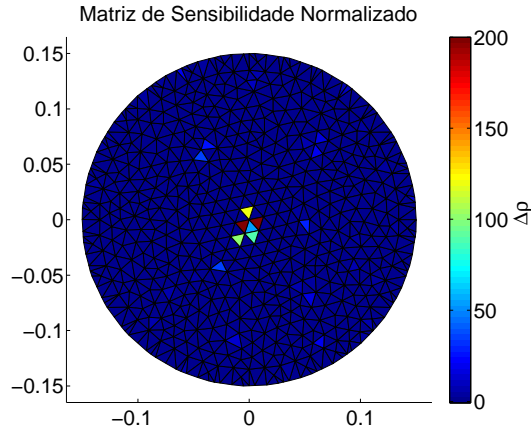

(a)

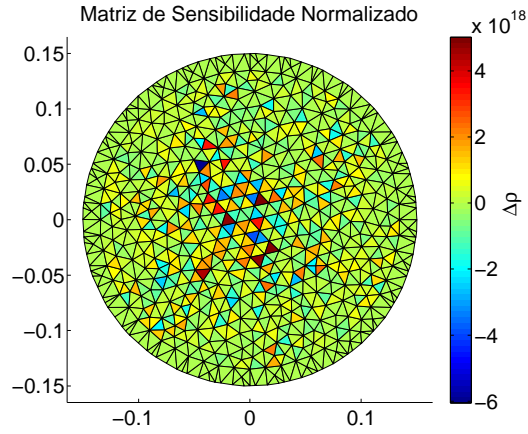

(b)

Figura 5.30: Distribuição de resistividade no centro com dados experimentais (a) com PL e (b) LU Decomposition

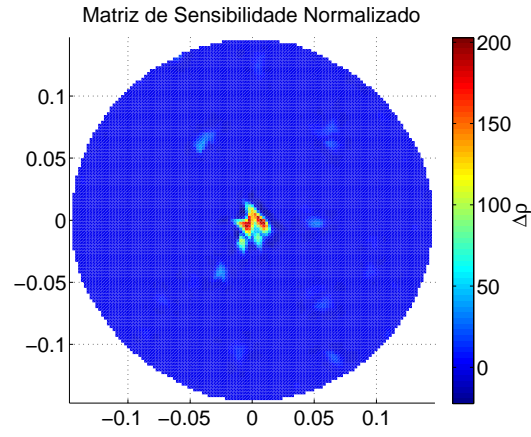

(a)

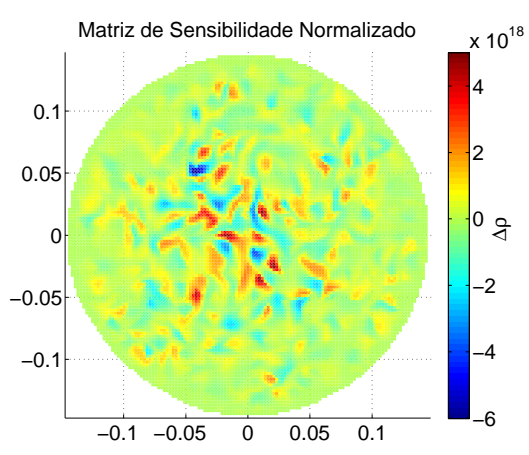

(b)

Figura 5.31: Imagem interpolada (a) com PL e (b) LU Decomposition

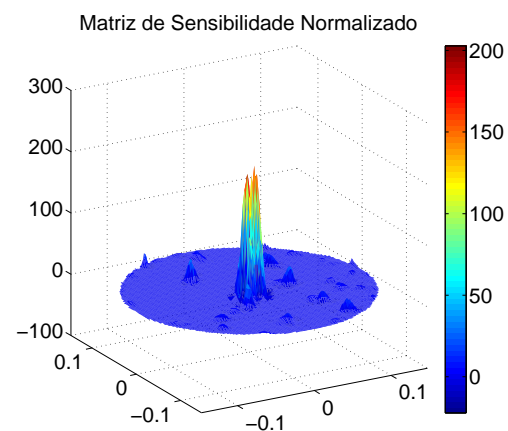

(a)

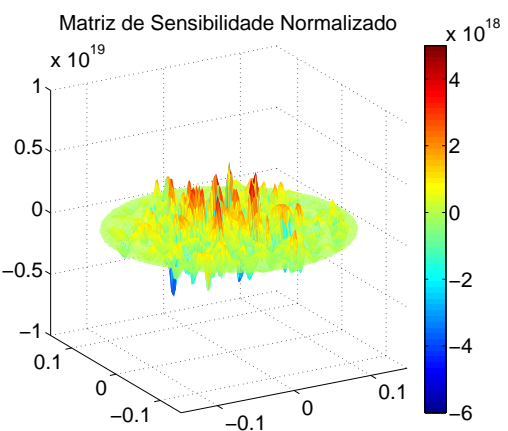

(b)

Figura 5.32: Imagem interpolada em perspectiva (a) com PL e (b) LU Decomposition 


\subsection{Resultados Implementando PLS e LU Decom- position no Método Gauss-Newton}

\subsubsection{Dados de Simulação Numérica}

Para a obtenção dos dados simulados quando o objeto está localizado perto da borda e no centro, usou-se a situação em que a resistividade é constante no domínio circular, exceto num objeto que fica perto da borda ou no centro, onde foi imposta uma resistividade muito maior. A resistividade do elemento pertur-

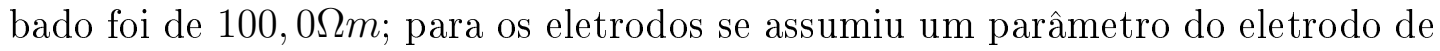
$0,02 \Omega m^{2}$ e para os demais elementos a resistividade foi de $20 \Omega m$.

Na solução do problema inverso, para o primeiro e segundo teste, utilizou-se uma estimativa inicial de resistividade homogênea de $10 \Omega m$ e $0,02 \Omega m^{2}$ para os elementos e o parâmetro do eletrodo, respectivamente.

Para a solução do problema direto foram usadas as malhas das figuras 4.9(a) e 4.10 (a) para o objeto perto da borda e perto do centro respetivamente. Para a solução do problema inverso foram usadas as malhas das figuras 4.9(b) e 4.10(b) para o objeto perto da borda e perto do centro. A continuação são mostradas as imagens geradas em cada teste ${ }^{3}$.

- Objeto perturbado perto da borda Parâmetro $\alpha=1,0 e^{-7}$

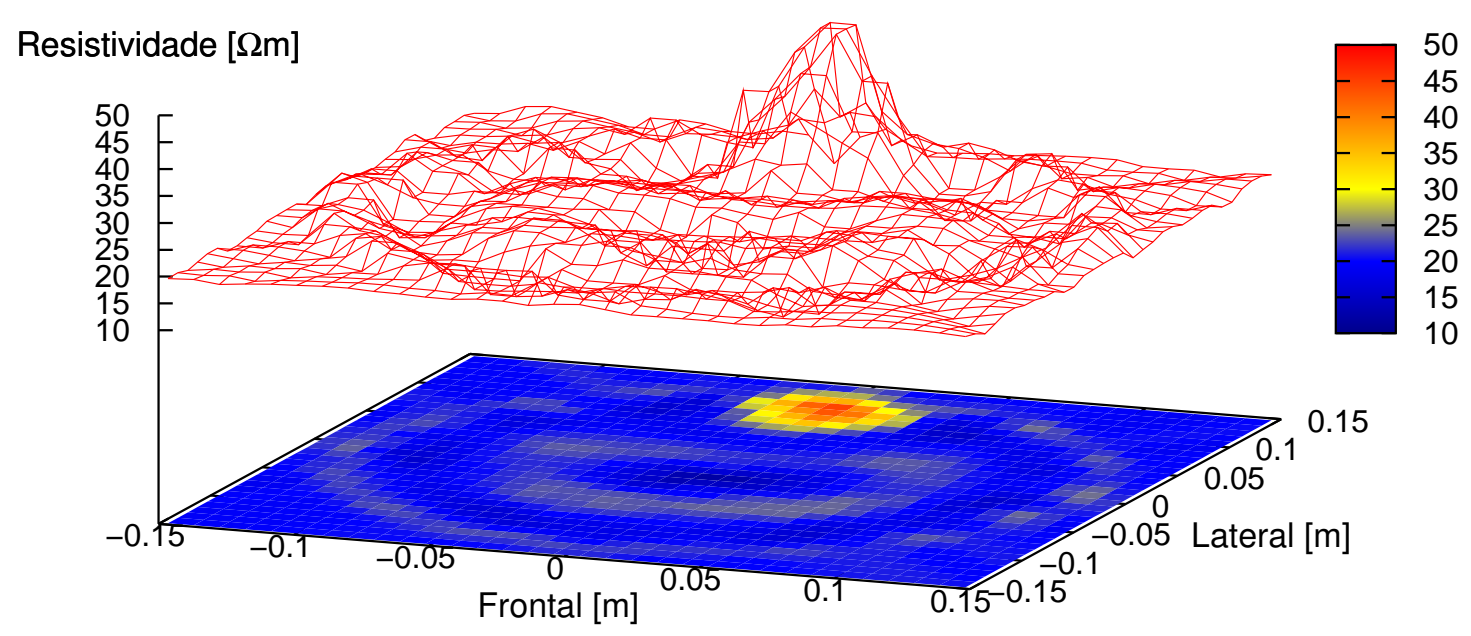

Figura 5.33: Obtenção de resistividade absoluta na borda com dados numéricos usando PLS para determinar o $\delta \rho$

\footnotetext{
${ }^{3}$ As imagens foram feitas no software "Matlab"e no "Gnuplot"onde o domínio circular está inscrito numa forma retangular. Alguns dos graficos são o resultado de uma interpolação adicional que é um filtro passa baixa e que deve ser ajustado cuidadosamente para não suavizar o resultado
} 


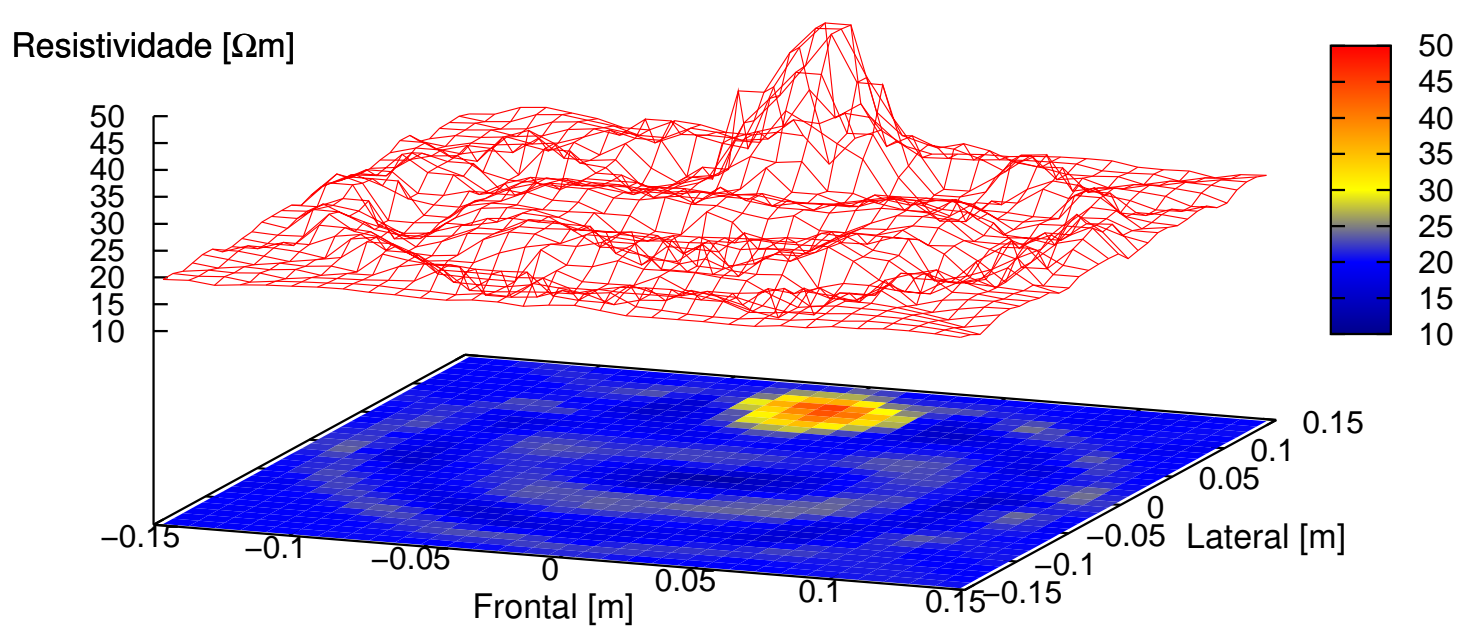

Figura 5.34: Obtenção de resistividade absoluta na borda com dados numéricos usando LU Decomposition para determinar o $\delta \rho$

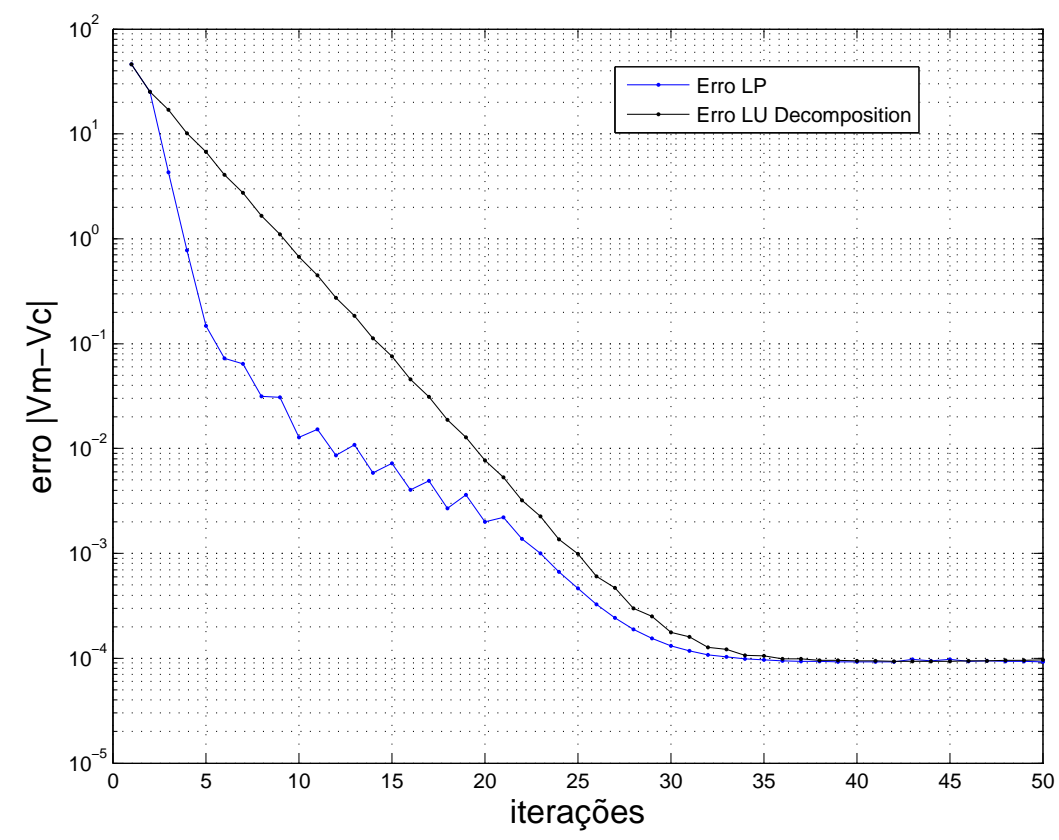

Figura 5.35: Índice de erro entre os potenciais elétricos medidos e os calculados 
- Objeto perturbado perto da borda Parâmetro $\alpha=1,0 e^{-9}$

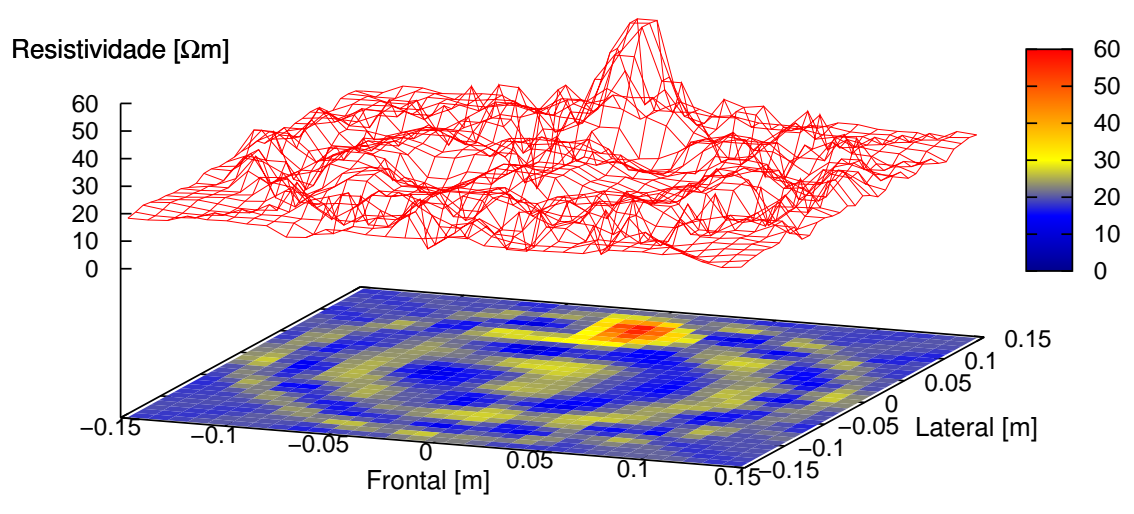

Figura 5.36: Obtenção de resistividade absoluta na borda com dados numéricos usando PLS para determinar o $\delta \rho$

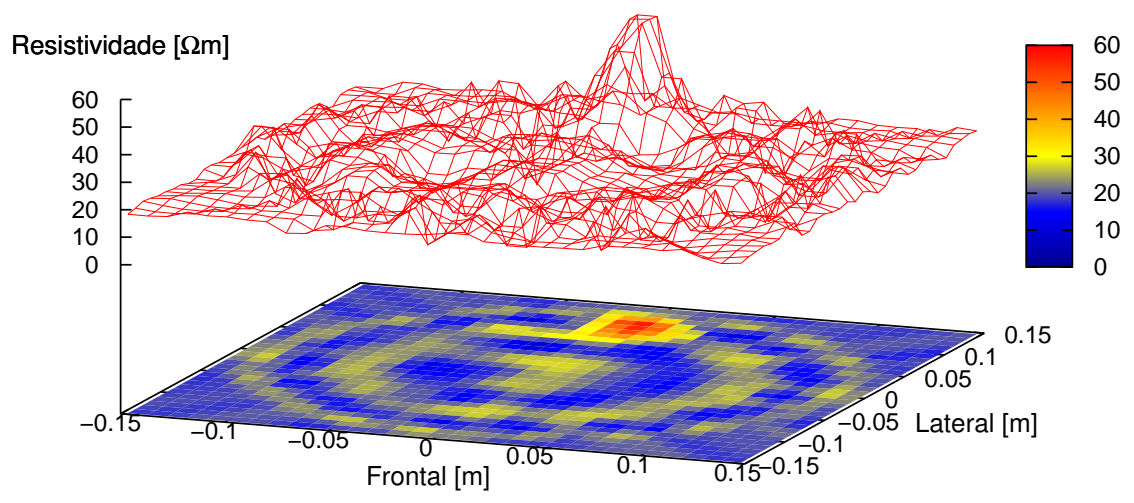

Figura 5.37: Obtenção de resistividade absoluta na borda com dados numéricos usando LU Decomposition para determinar o $\delta \rho$

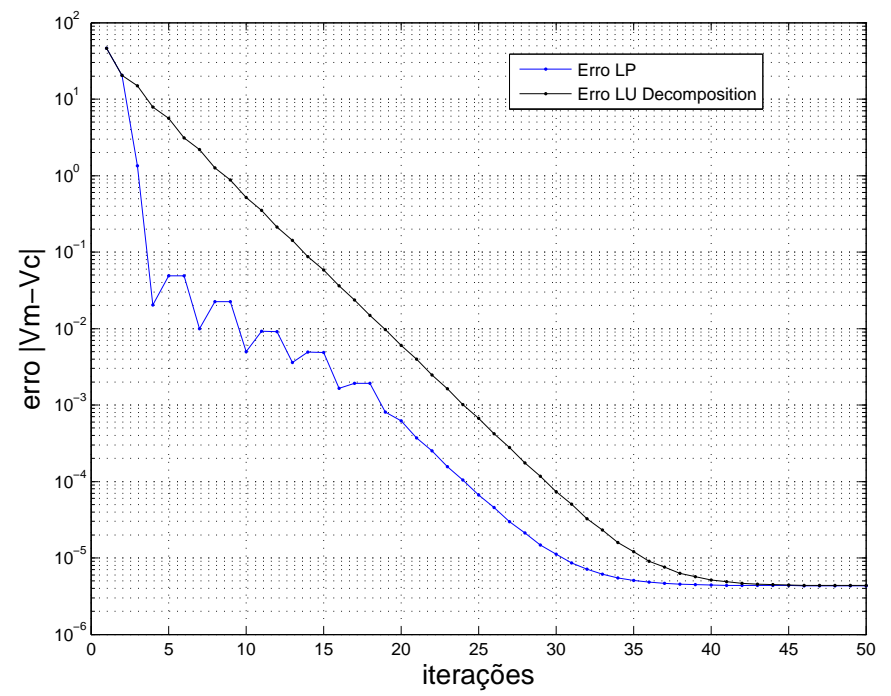

Figura 5.38: Índice de erro entre os potenciais elétricos medidos e os calculados 
- Objeto perturbado perto da borda Parâmetro $\alpha=1,0 e^{-10}$

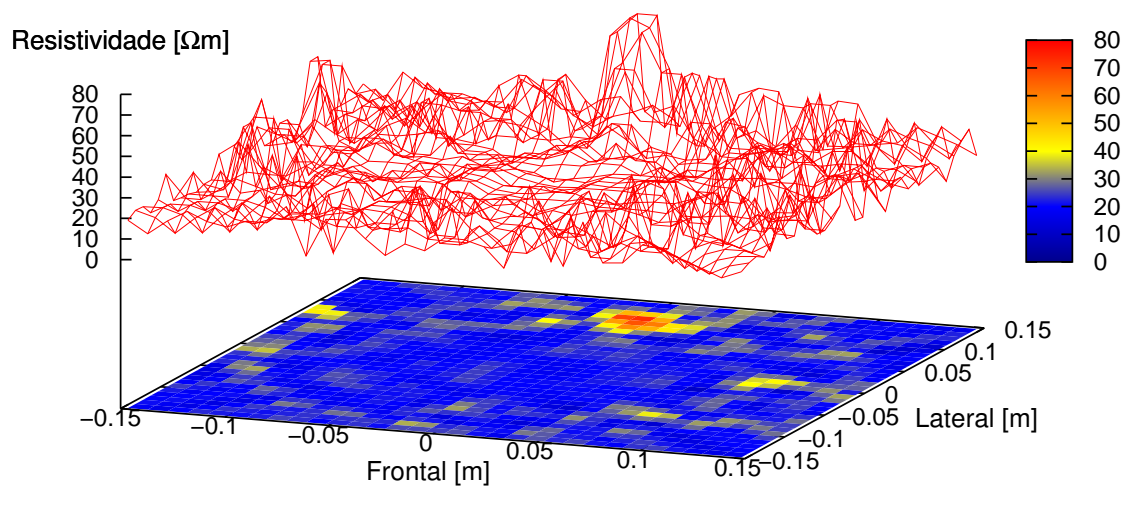

Figura 5.39: Obtenção de resistividade absoluta na borda com dados numéricos usando PLS para determinar o $\delta \rho$

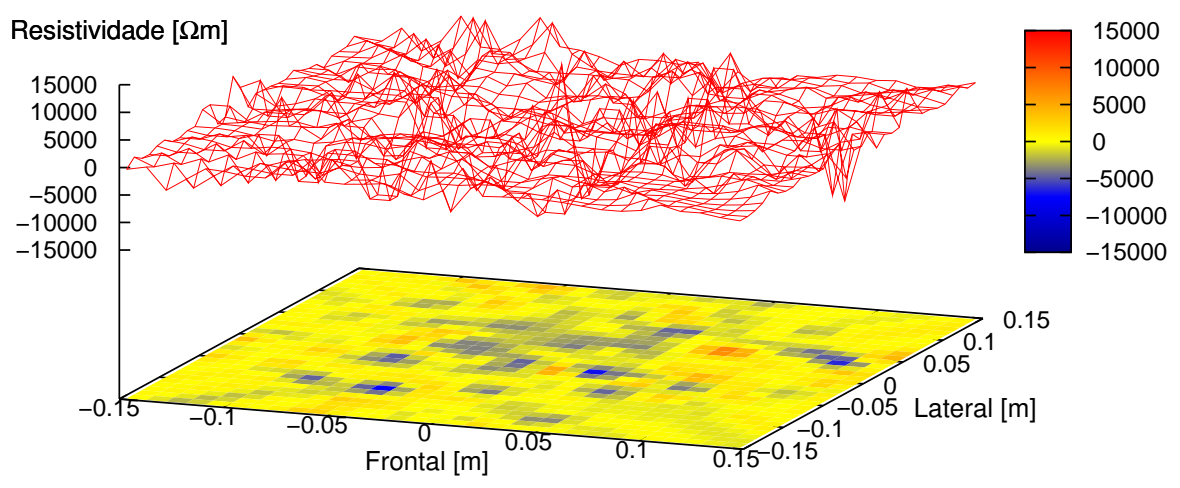

Figura 5.40: Obtenção de resistividade absoluta na borda com dados numéricos usando LU Decomposition para determinar o $\delta \rho$

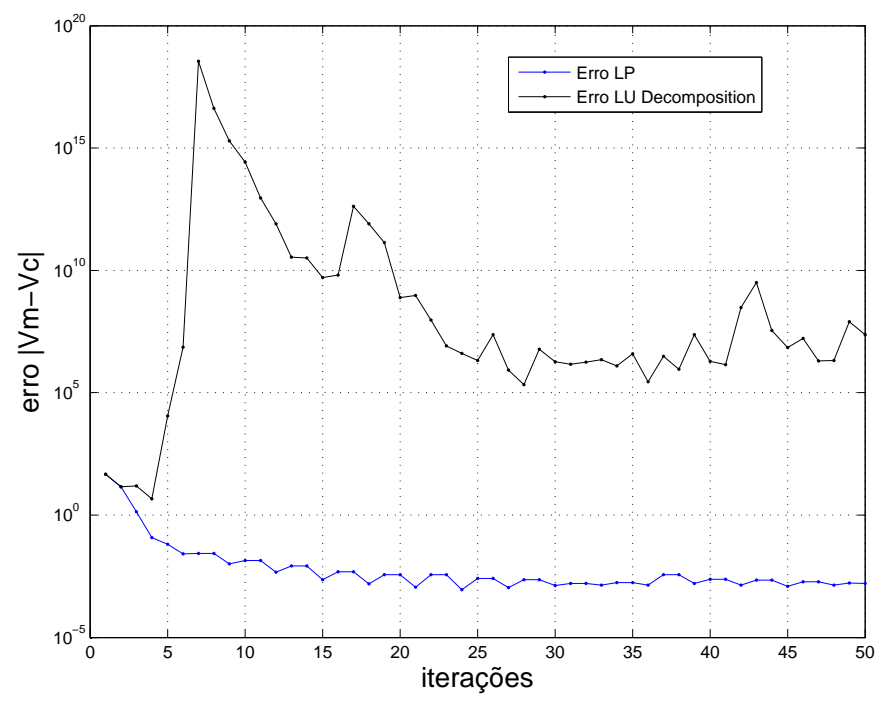

Figura 5.41: Índice de erro entre os potenciais elétricos medidos e os calculados 
- Objeto perturbado perto do centro Parâmetro $\alpha=\mathbf{1}, 0 e^{-7}$

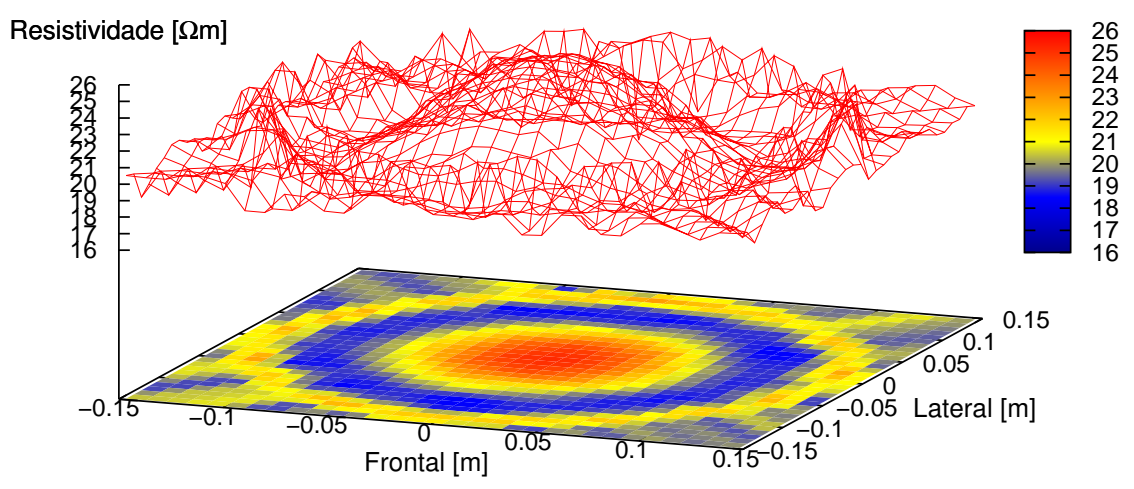

Figura 5.42: Obtenção de resistividade absoluta no centro com dados numéricos usando PLS para determinar o $\delta \rho$

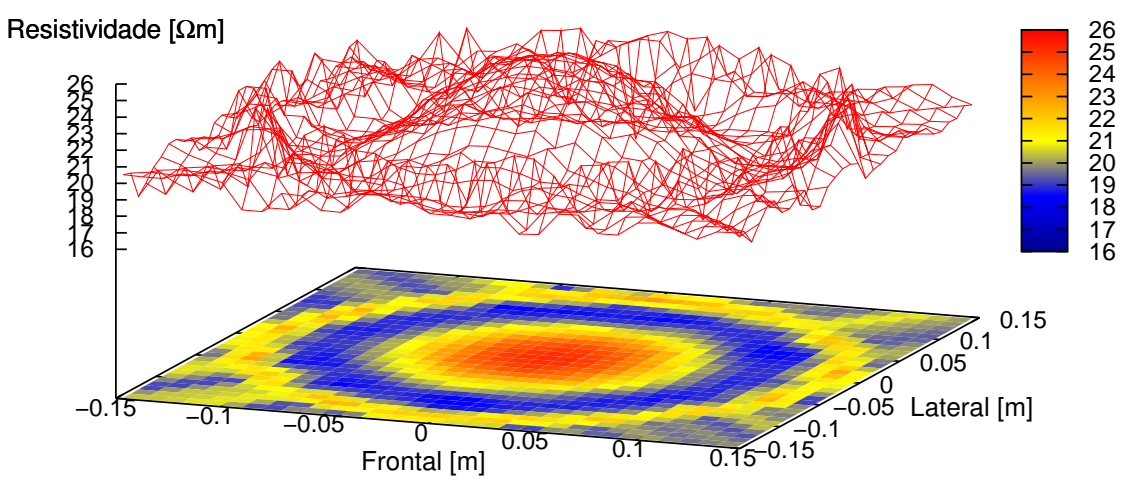

Figura 5.43: Obtenção de resistividade absoluta no centro com dados numéricos usando LU Decomposition para determinar o $\delta \rho$

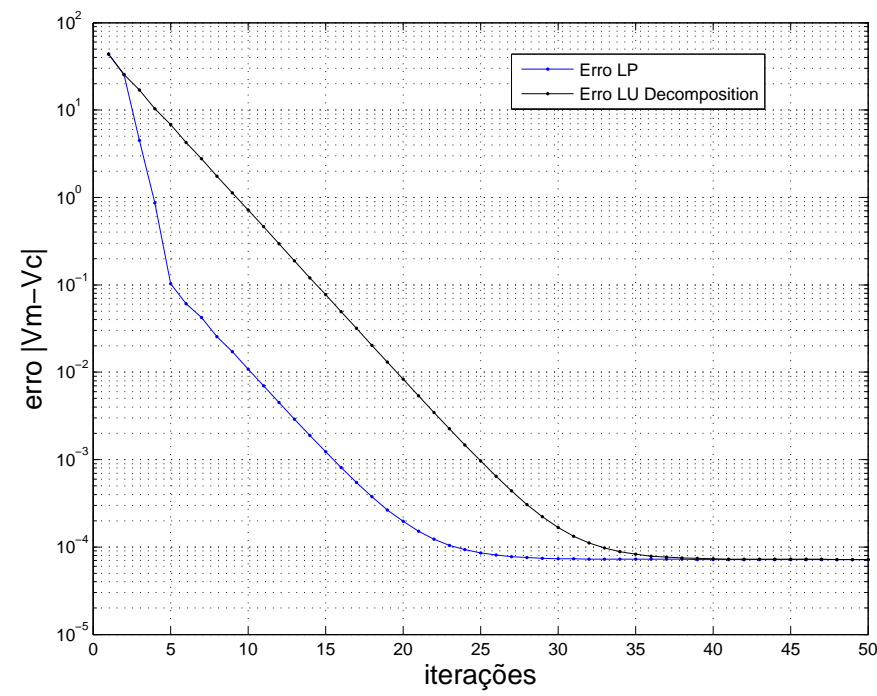

Figura 5.44: Índice de erro entre os potenciais elétricos medidos e os calculados 
- Objeto perturbado perto do centro Parâmetro $\alpha=\mathbf{1 , 0 e ^ { - 9 }}$

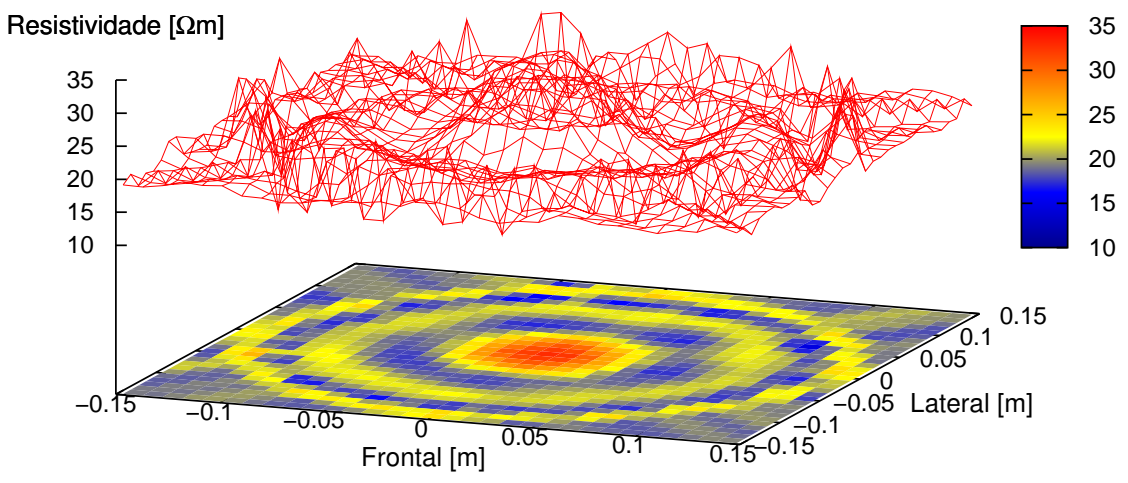

Figura 5.45: Obtenção de resistividade absoluta no centro com dados numéricos usando PLS para determinar o $\delta \rho$

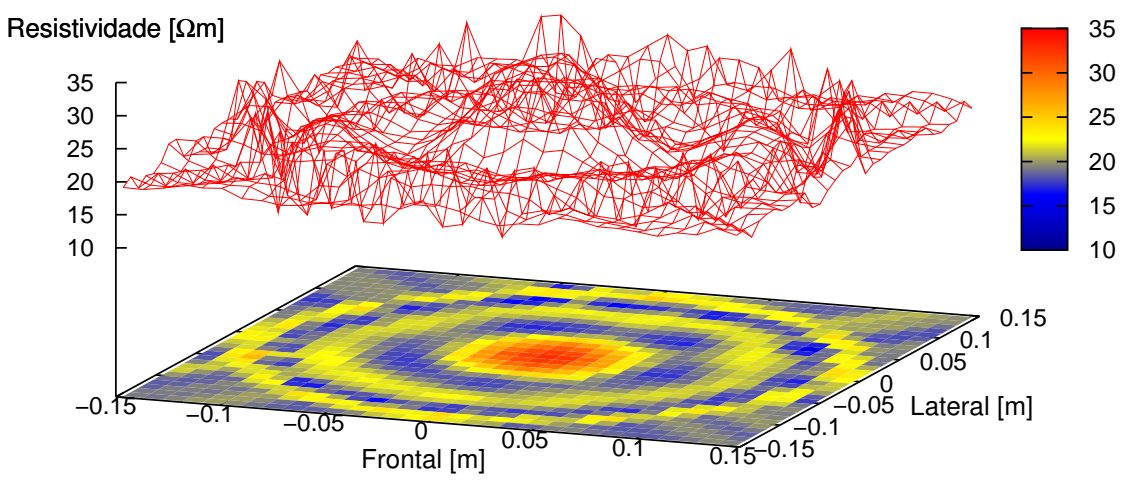

Figura 5.46: Obtenção de resistividade absoluta no centro com dados numéricos usando LU Decomposition para determinar o $\delta \rho$

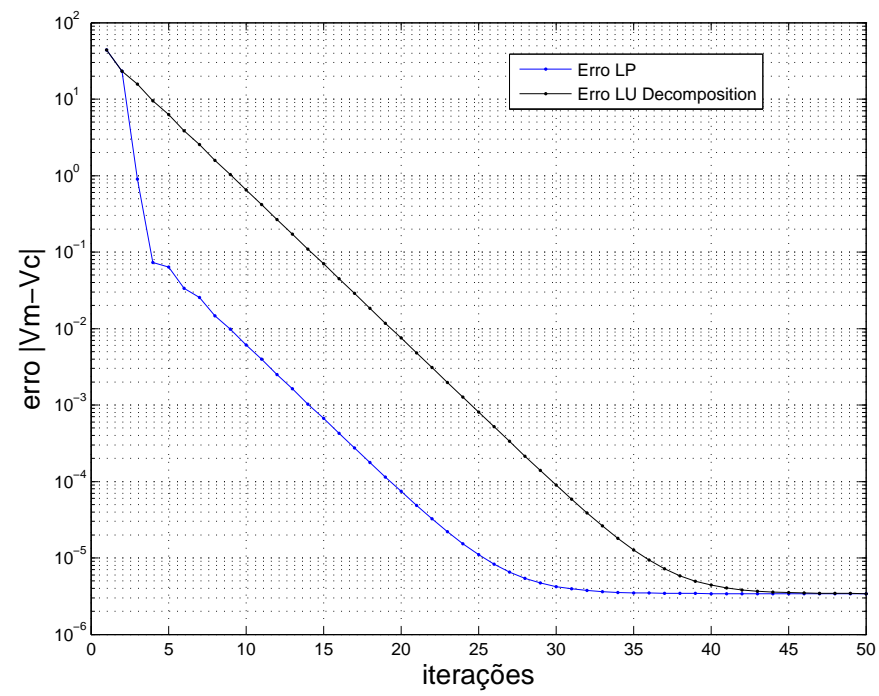

Figura 5.47: Índice de erro entre os potenciais elétricos medidos e os calculados 
- Objeto perturbado perto do centro Parâmetro $\alpha=3,0 e^{-11}$

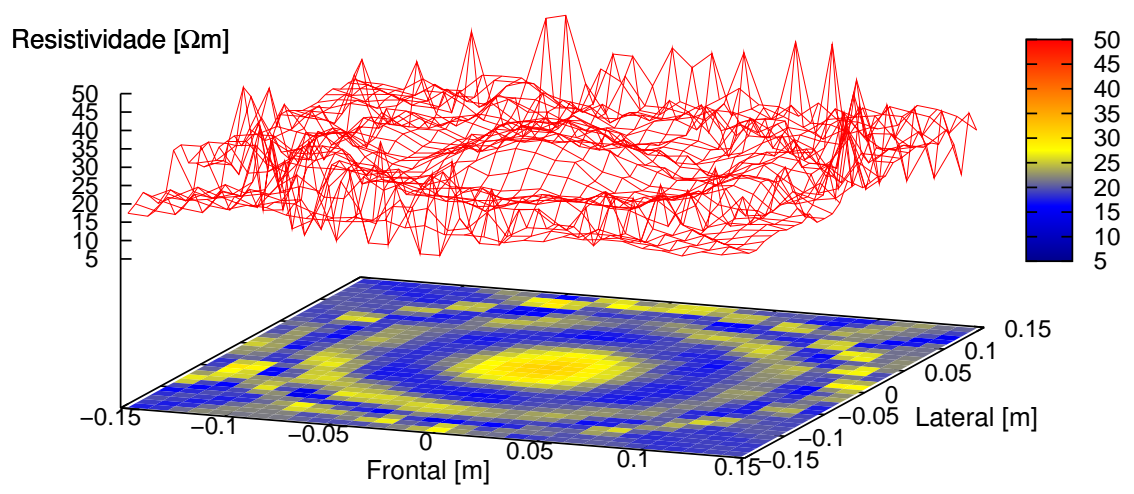

Figura 5.48: Obtenção de resistividade absoluta no centro com dados numéricos usando PLS para determinar o $\delta \rho$

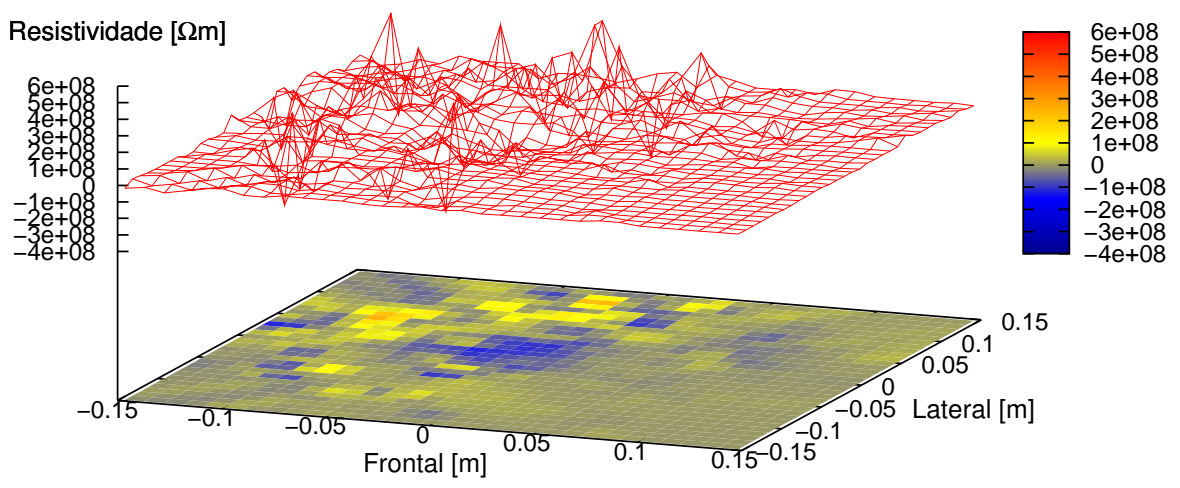

Figura 5.49: Obtenção de resistividade absoluta no centro com dados numéricos usando LU Decomposition para determinar o $\delta \rho$

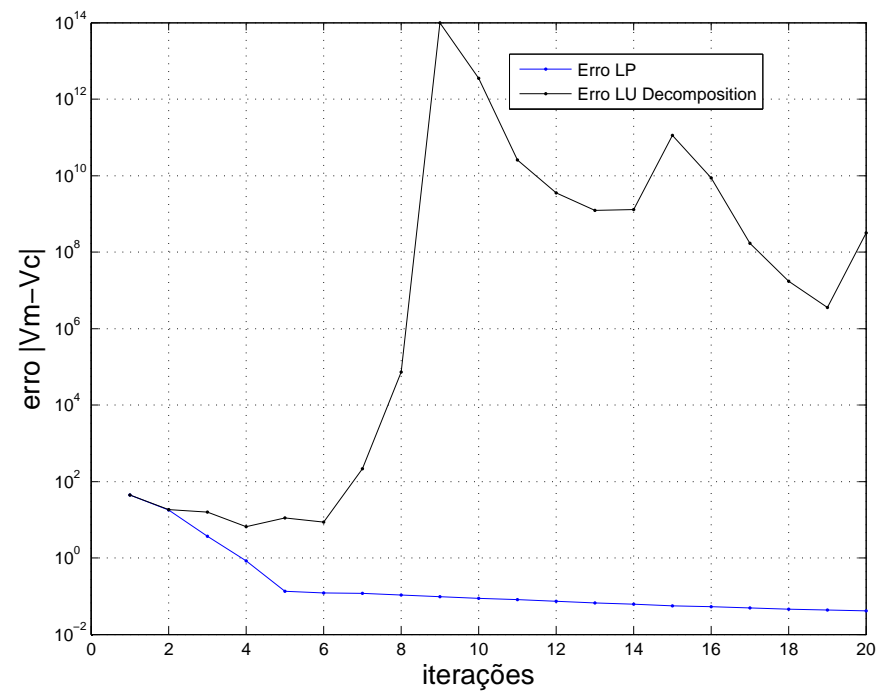

Figura 5.50: Índice de erro entre os potenciais elétricos medidos e os calculados 


\subsection{Comparação das Imagens Obtidas com LU De- composition e PLS Utilizando RIM}

\subsubsection{Dados de Simulação Numérica}

Os dados utilizados quando é utilizada a restrição de inclinação máxima de resistividade RIM são os mesmos mencionados na seção 5.3, desta vez a restrição RIM vai ser acrescentada no sistema de restrições como mostrado na equação 3.56

- Objeto perturbado perto da borda Parâmetro $\alpha=1,0 e^{-7}$

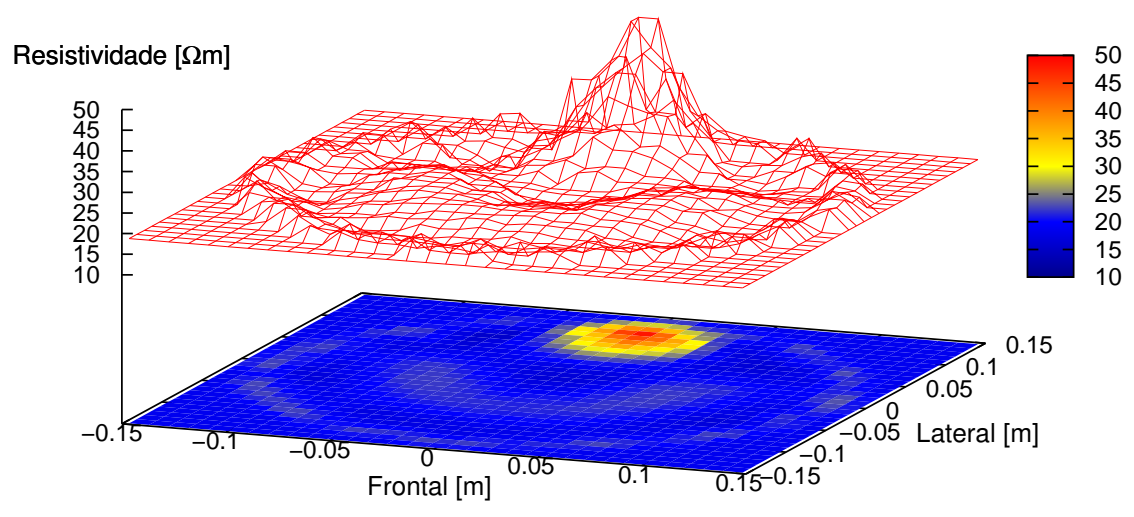

Figura 5.51: Obtenção de resistividade absoluta na borda com dados numéricos usando PLS e RIM para determinar o $\delta \rho$

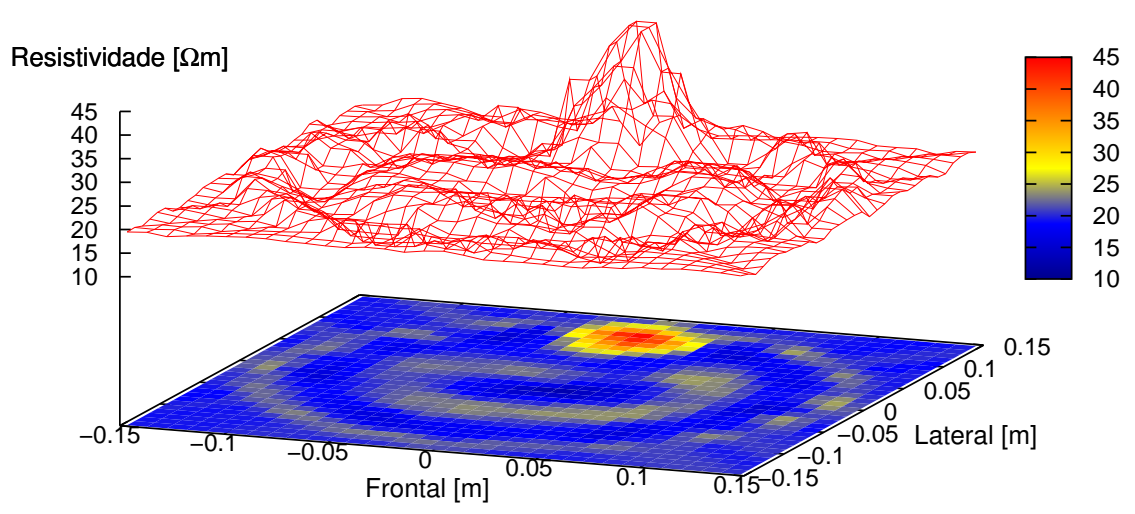

Figura 5.52: Obtenção de resistividade absoluta no centro com dados numéricos usando LU Decomposition para determinar o $\delta \rho$ 


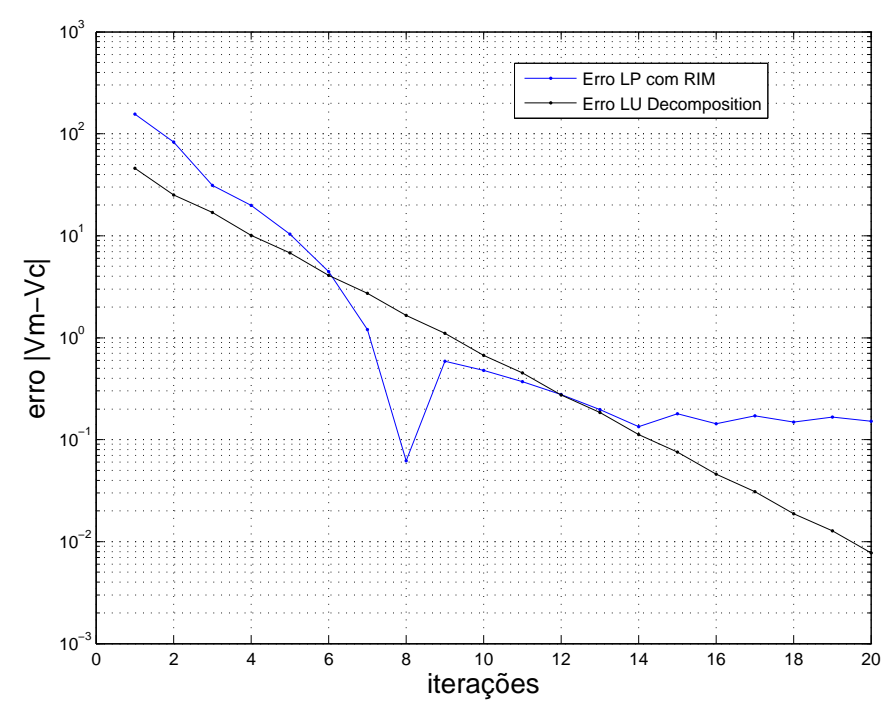

Figura 5.53: Índice de erro entre os potenciais elétricos medidos e os calculados

- Objeto perturbado perto da borda Parâmetro $\alpha=\mathbf{1}, 0 e^{-9}$

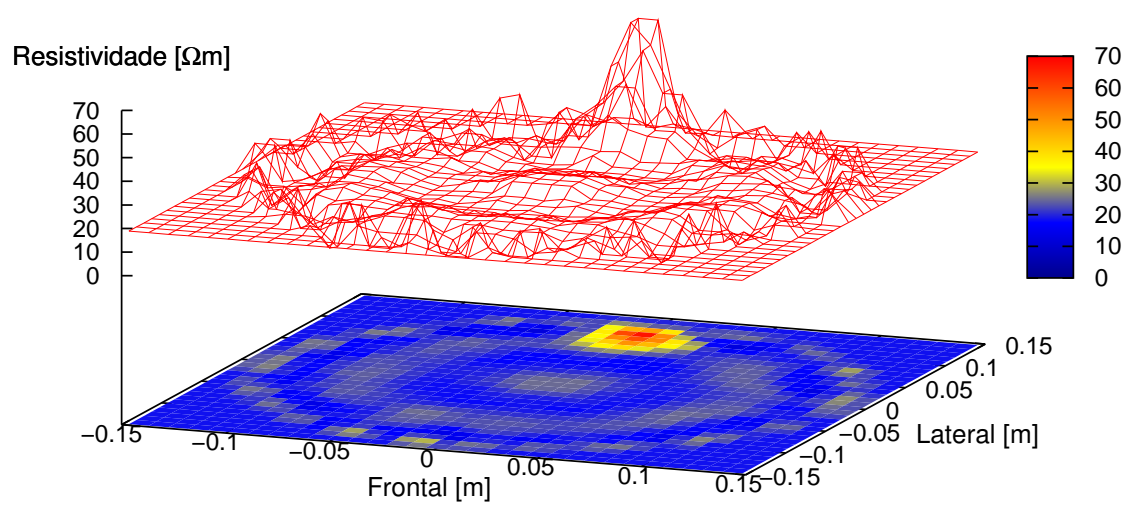

Figura 5.54: Obtenção de resistividade absoluta na borda com dados numéricos usando PLS e RIM para determinar o $\delta \rho$

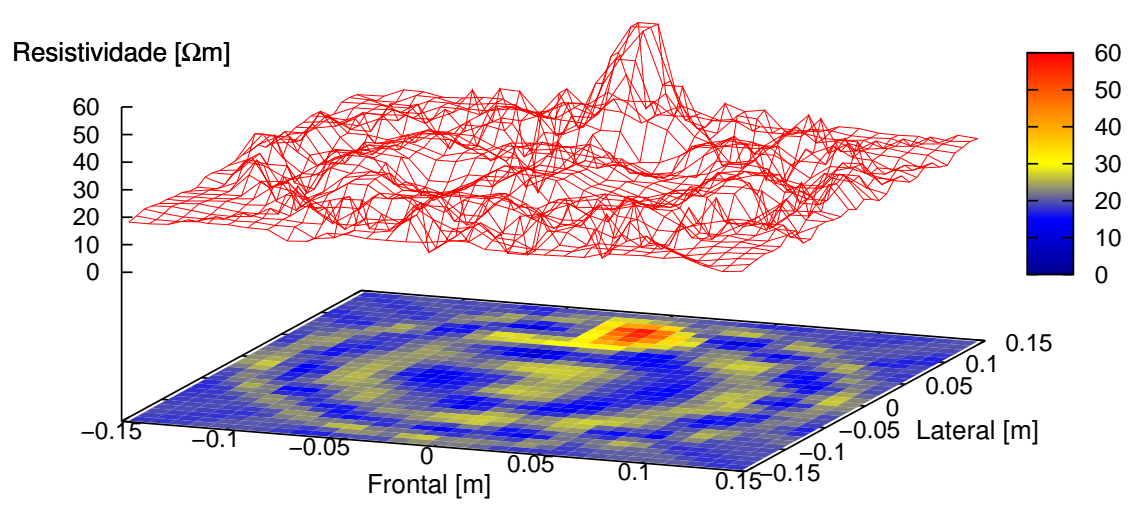

Figura 5.55: Obtenção de resistividade absoluta no centro com dados numéricos usando LU Decomposition para determinar o $\delta \rho$ 


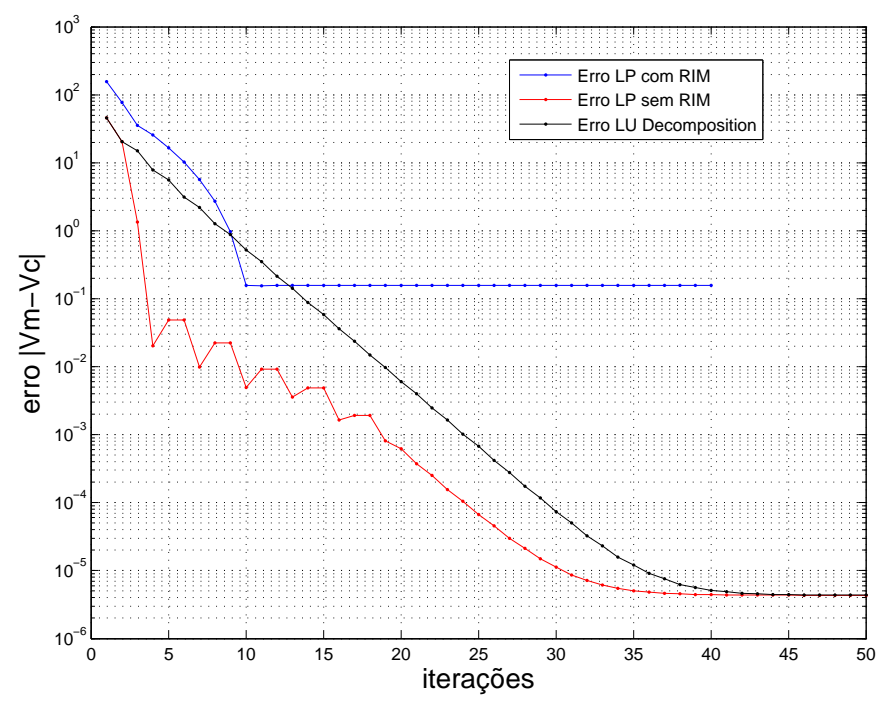

Figura 5.56: Índice de erro entre os potenciais elétricos medidos e os calculados

- Objeto perturbado perto da borda Parâmetro $\alpha=\mathbf{1}, 0 e^{-10}$

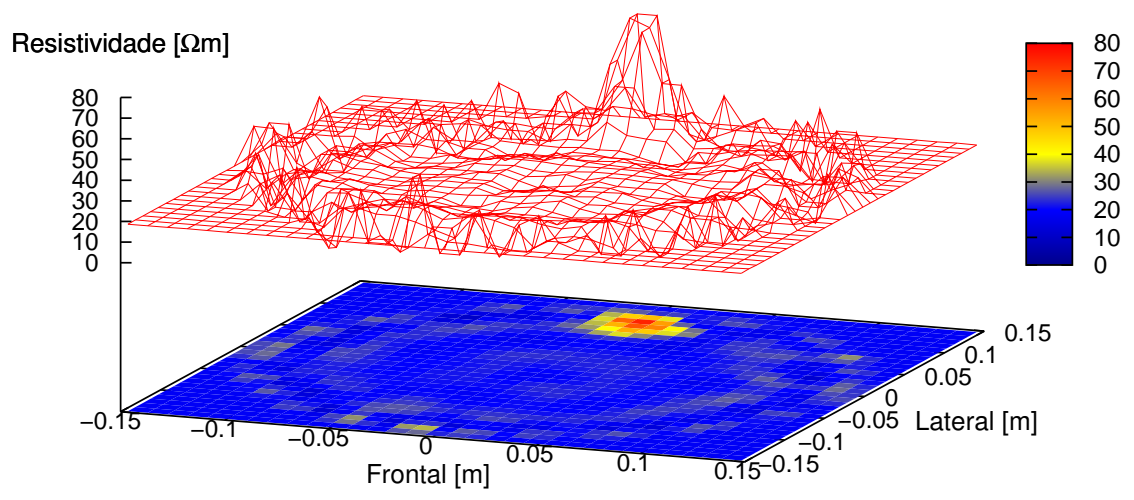

Figura 5.57: Obtenção de resistividade absoluta na borda com dados numéricos usando PLS e RIM para determinar o $\delta \rho$

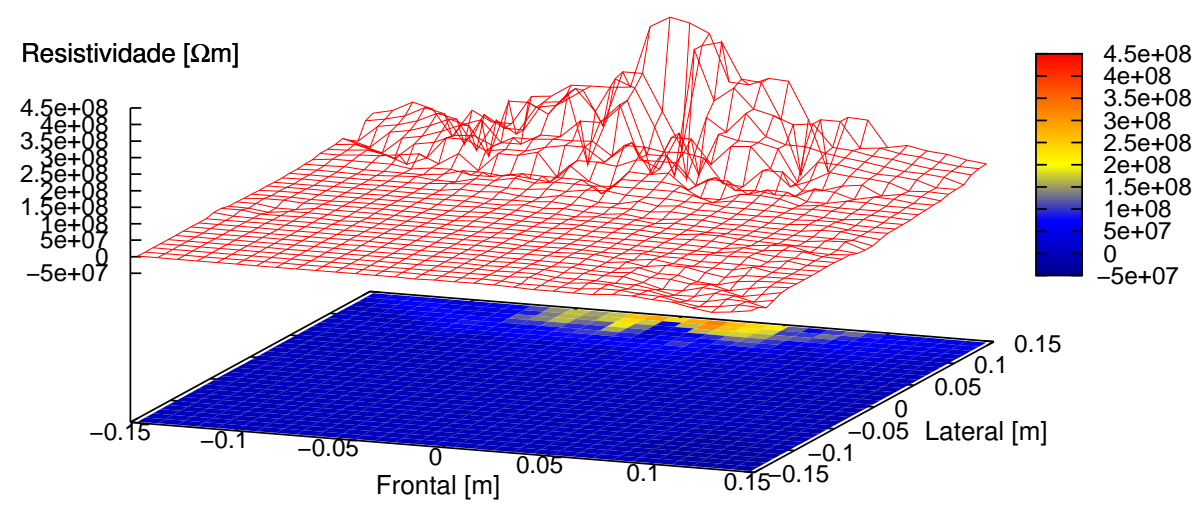

Figura 5.58: Obtenção de resistividade absoluta no centro com dados numéricos usando LU Decomposition para determinar o $\delta \rho$ 


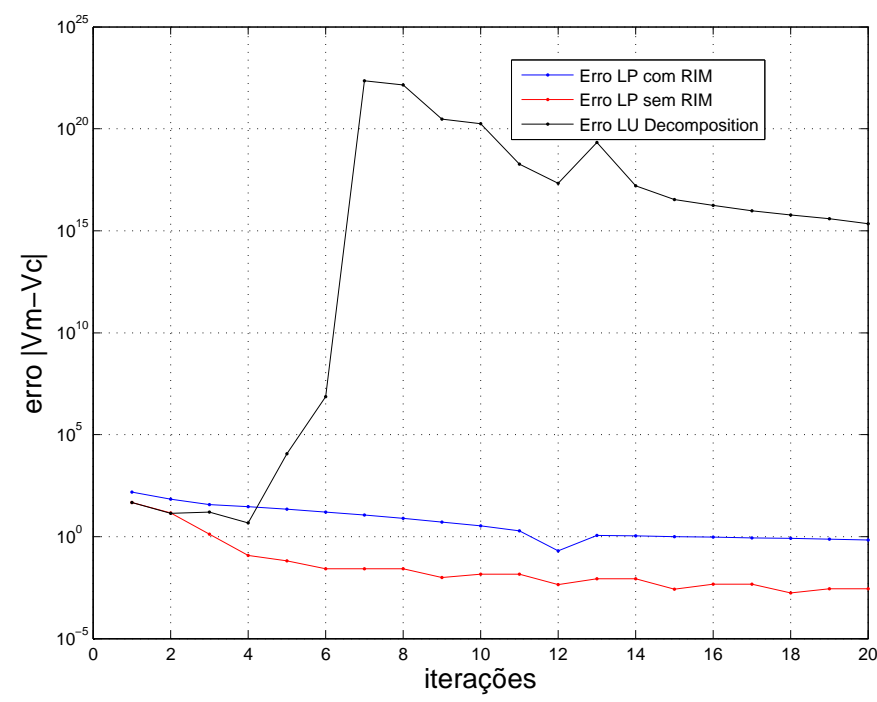

Figura 5.59: Índice de erro entre os potenciais elétricos medidos e os calculados

- Objeto perturbado perto do centro Parâmetro $\alpha=\mathbf{1}, 0 e^{-7}$

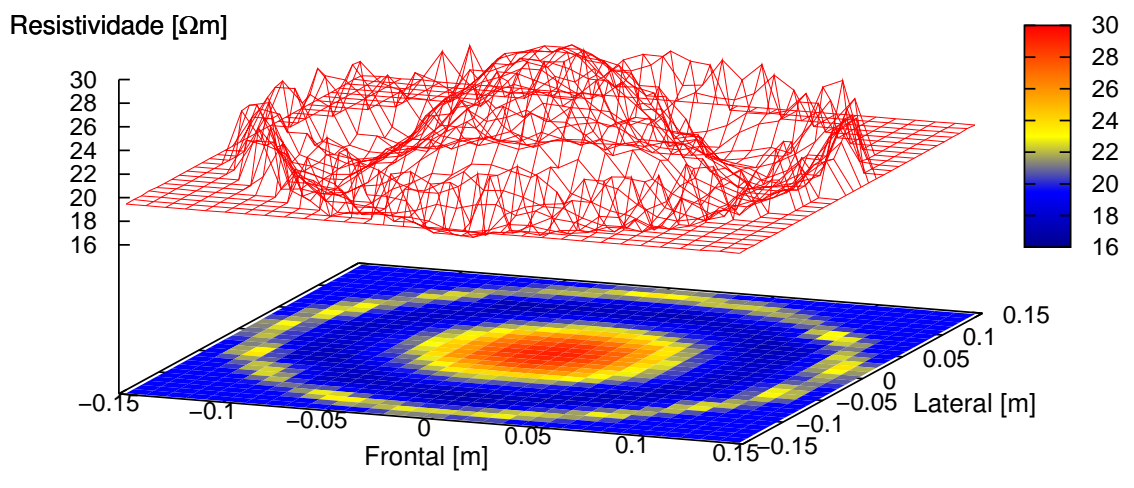

Figura 5.60: Obtenção de resistividade absoluta no centro com dados numéricos usando PLS e RIM para determinar o $\delta \rho$

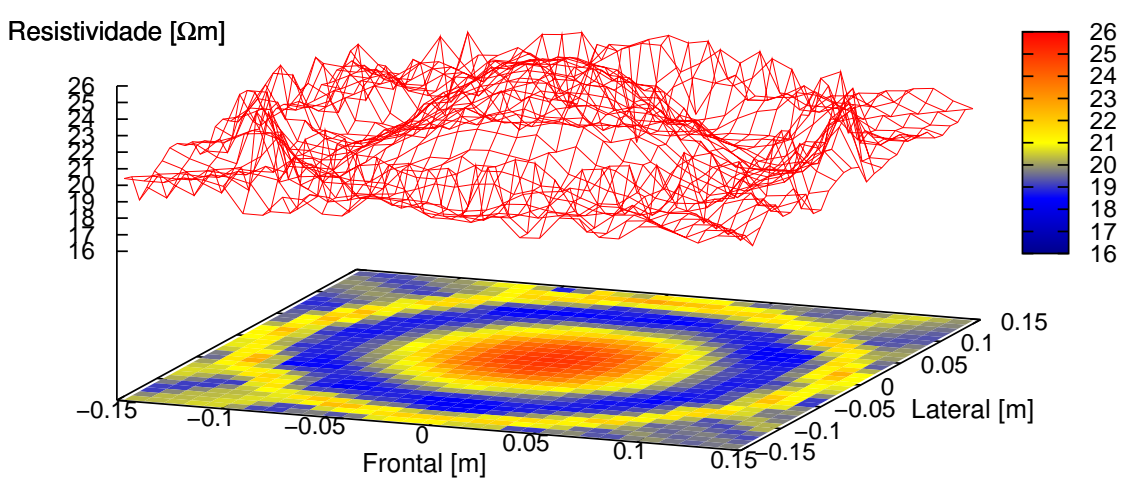

Figura 5.61: Obtenção de resistividade absoluta no centro com dados numéricos usando LU Decomposition para determinar o $\delta \rho$ 


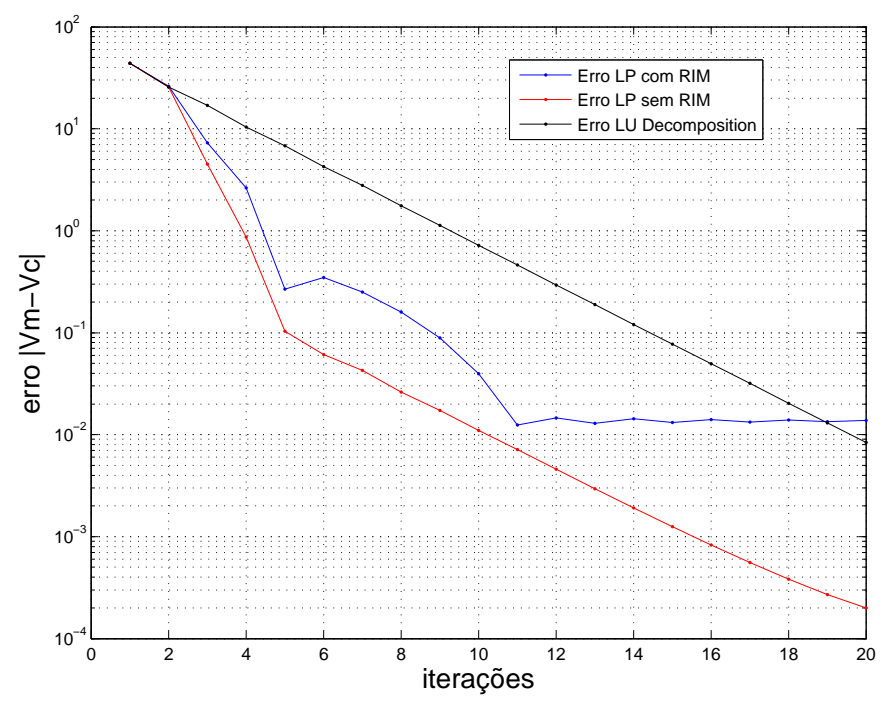

Figura 5.62: Índice de erro entre os potenciais elétricos medidos e os calculados

- Objeto perturbado perto do centro Parâmetro $\alpha=\mathbf{1}, 0 e^{-9}$

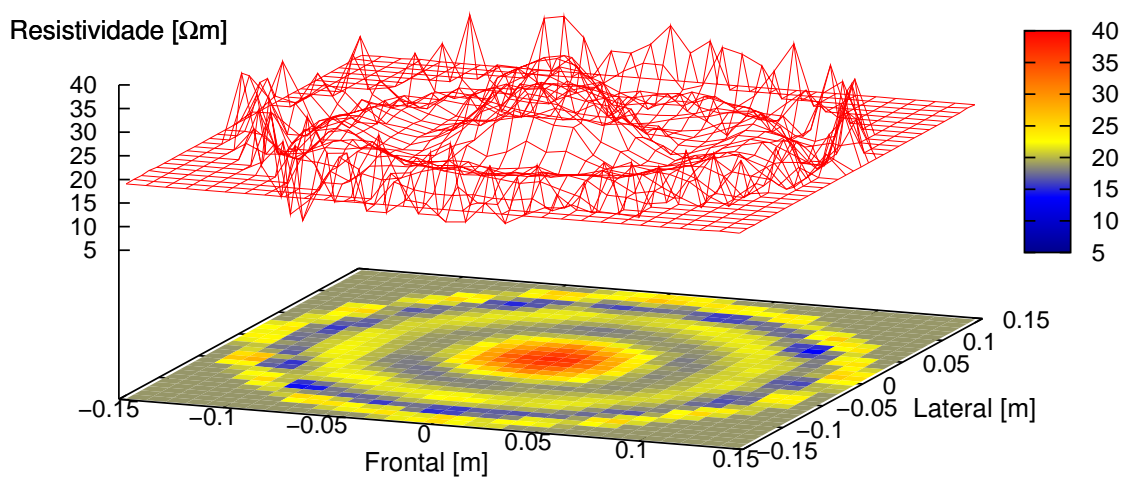

Figura 5.63: Obtenção de resistividade absoluta no centro com dados numéricos usando PLS e RIM para determinar o $\delta \rho$

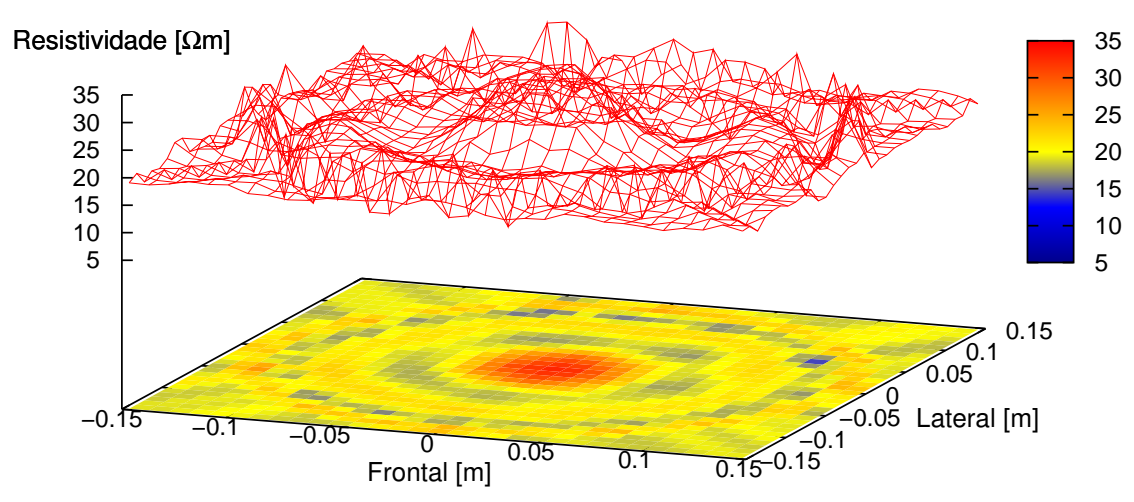

Figura 5.64: Obtenção de resistividade absoluta no centro com dados numéricos usando LU Decomposition para determinar o $\delta \rho$ 


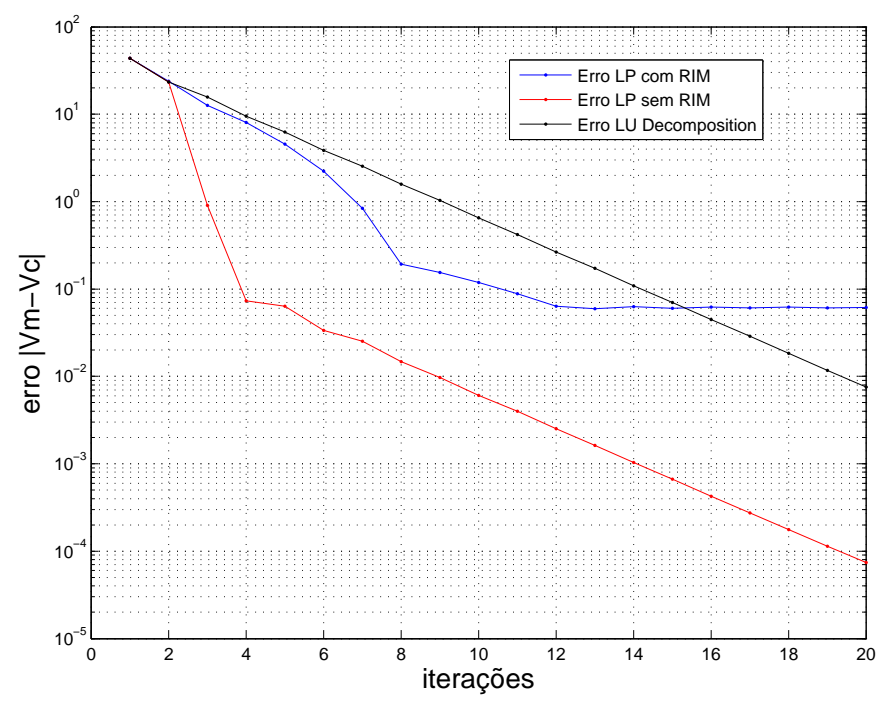

Figura 5.65: Índice de erro entre os potenciais elétricos medidos e os calculados

- Objeto perturbado perto do centro Parâmetro $\alpha=3,0 e^{-11}$

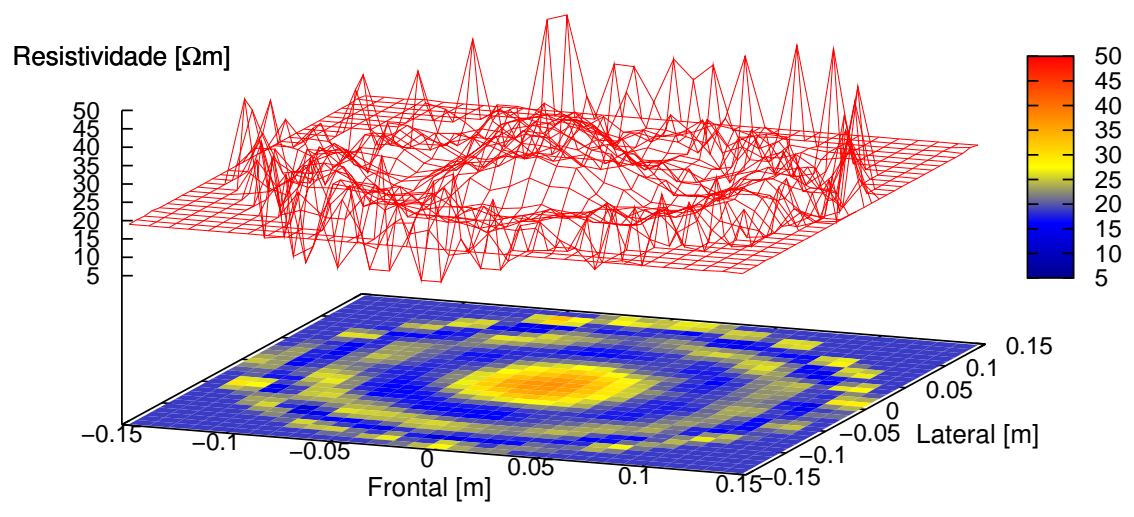

Figura 5.66: Obtenção de resistividade absoluta no centro com dados numéricos usando PLS e RIM para determinar o $\delta \rho$

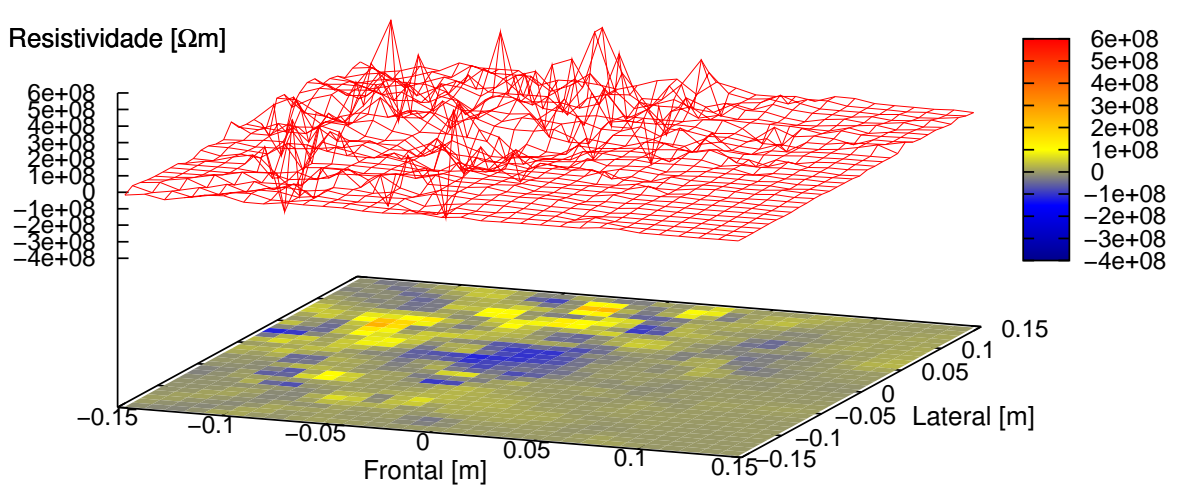

Figura 5.67: Obtenção de resistividade absoluta no centro com dados numéricos usando LU Decomposition para determinar o $\delta \rho$ 


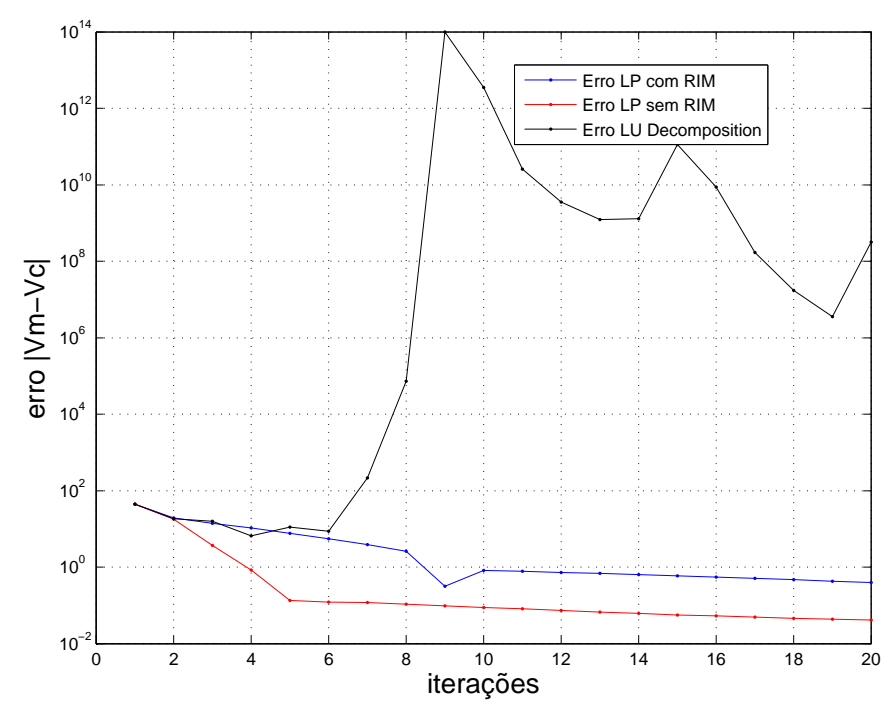

Figura 5.68: Índice de erro entre os potenciais elétricos medidos e os calculados

\subsubsection{Dados de Bancada Experimental}

Para a obtenção dos dados de bancada experimental, foram feitos dos tipos de testes. No primeiro teste, colocou-se o objeto em duas posições diferentes como mostrado nas figuras 4.2(a) e 4.2(b). O parâmetro do eletrodo foi assumido igual a $0,02 \Omega m^{2}$, para a solução salina a resistividade foi medida com o condutivímetro e foi igual a $2,77 \Omega m$. Na solução do problema inverso utilizou-se uma estimativa inicial de resistividade homogênea de $1 \Omega m$ e $0,02 \Omega m^{2}$ para os elementos e o parâmetro do eletrodo respectivamente.

Para o segundo teste foram gerados objetos similares as geometrias do pulmão e o coração como mostrado nas figuras 4.3(a) e 4.3(b). A resistividade do objeto similar ao pulmão foi de $3,8 \Omega m$ e do objeto similar ao coração foi de $0,98 \Omega m$, a resistividade da solução salina foi de $2,27 \Omega m$. Na solução do problema inverso utilizou-se uma estimativa inicial de resistividade homogênea de $1 \Omega m$ para os elementos e de $0,02 \Omega m^{2}$ para o parâmetro do eletrodo. A malha utilizada para resolver o problema inverso tanto do objeto de acrílico como dos objetos de agar é mostrada na figura 4.12 
- Objeto de acrílico perto da borda Parâmetro $\alpha=1,0 e^{-4}$

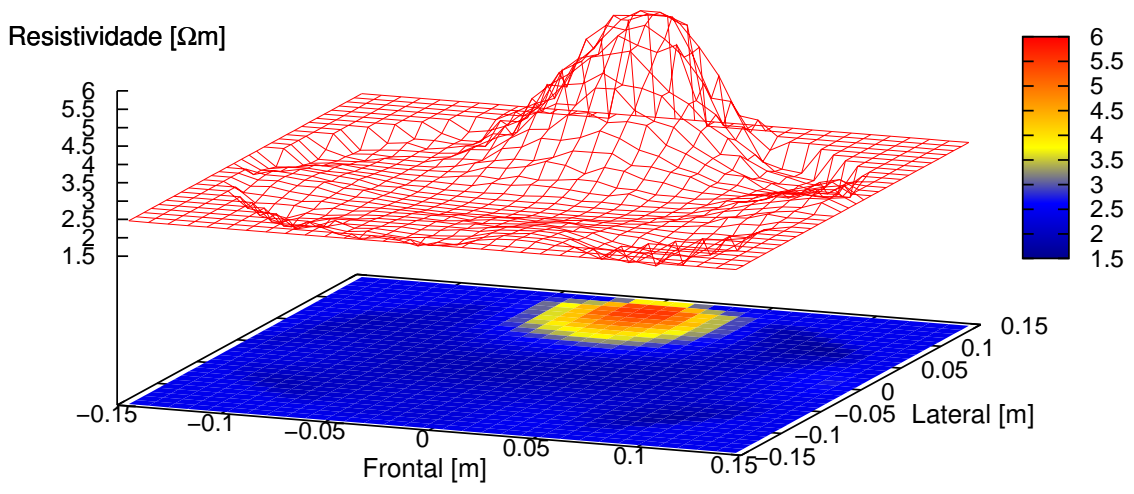

Figura 5.69: Obtenção de resistividade absoluta na borda com dados experimentais usando PLS e RIM para determinar o $\delta \rho$

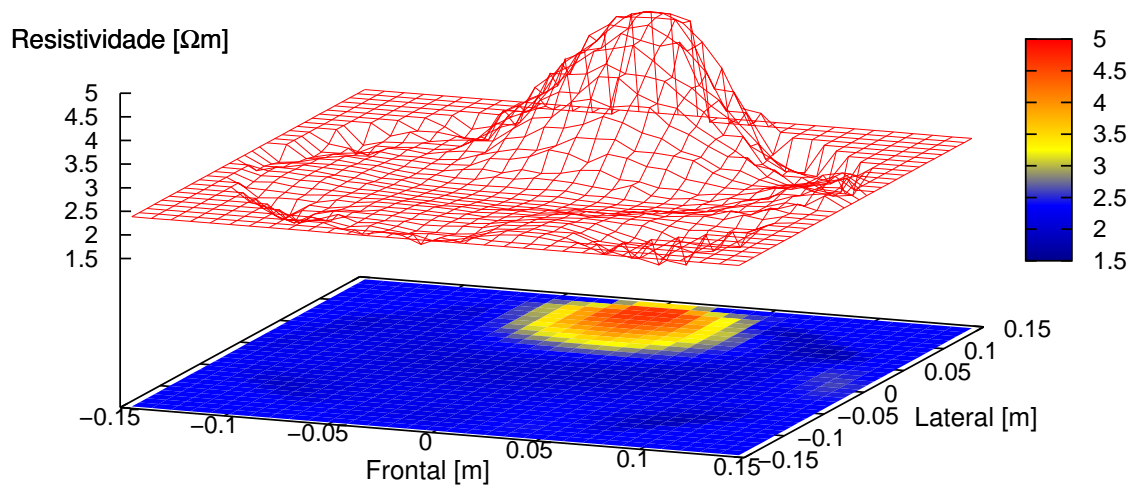

Figura 5.70: Obtenção de resistividade absoluta na borda com dados experimentais usando LU Decomposition para determinar o $\delta \rho$

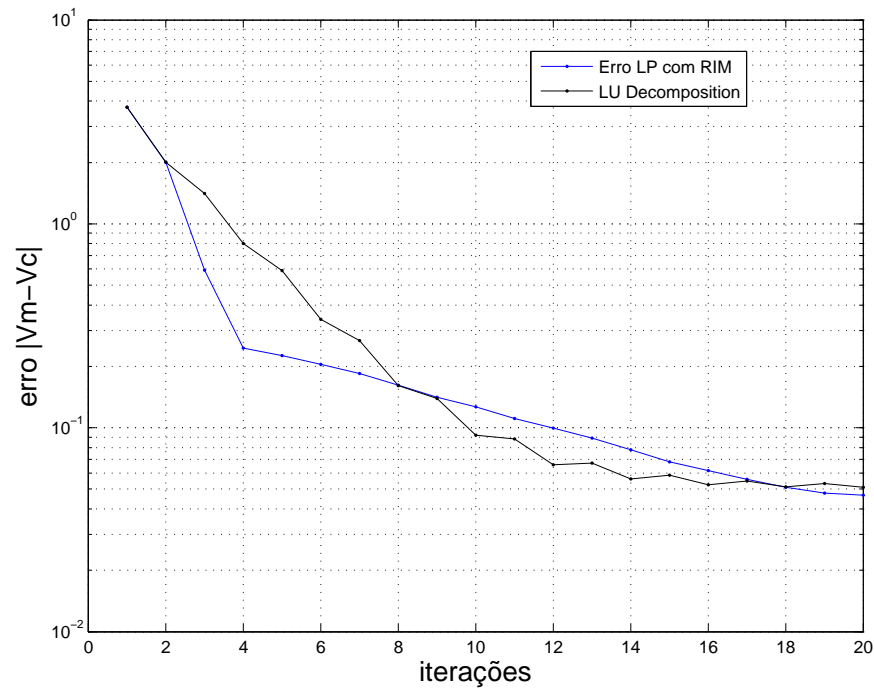

Figura 5.71: Índice de erro entre os potenciais elétricos medidos e os calculados 
- Objeto de acrílico perto da borda Parâmetro $\alpha=1,0 e^{-5}$

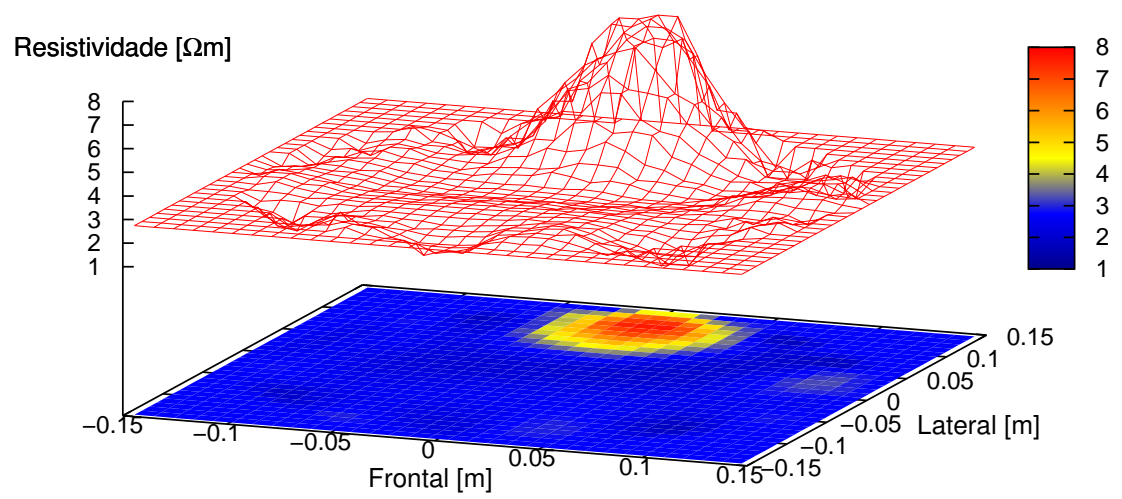

Figura 5.72: Obtenção de resistividade absoluta na borda com dados experimentais usando PLS e RIM para determinar o $\delta \rho$

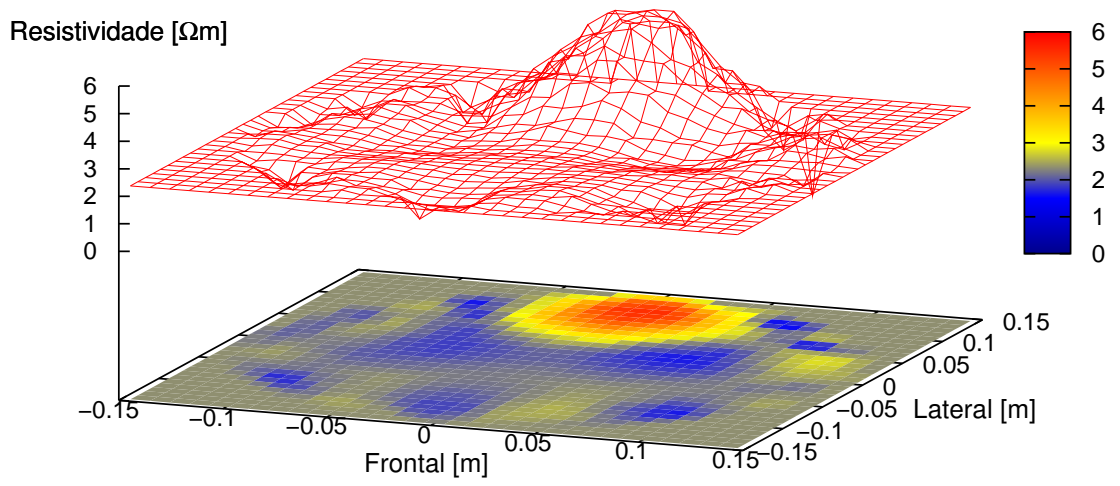

Figura 5.73: Obtenção de resistividade absoluta na borda com dados experimentais usando LU Decomposition para determinar o $\delta \rho$

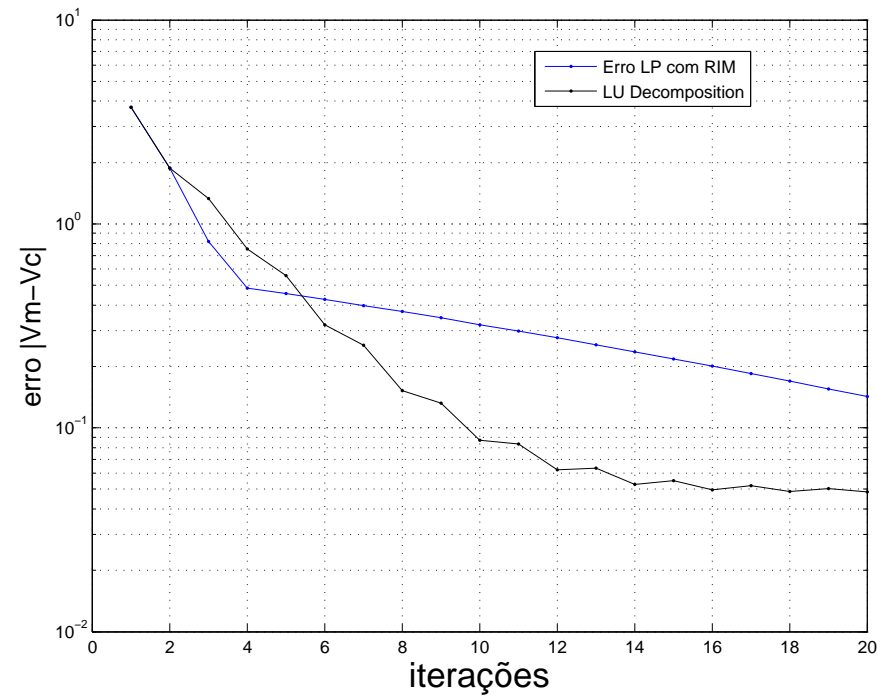

Figura 5.74: Índice de erro entre os potenciais elétricos medidos e os calculados 
- Objeto de acrílico perto da borda Parâmetro $\alpha=1,0 e^{-6}$

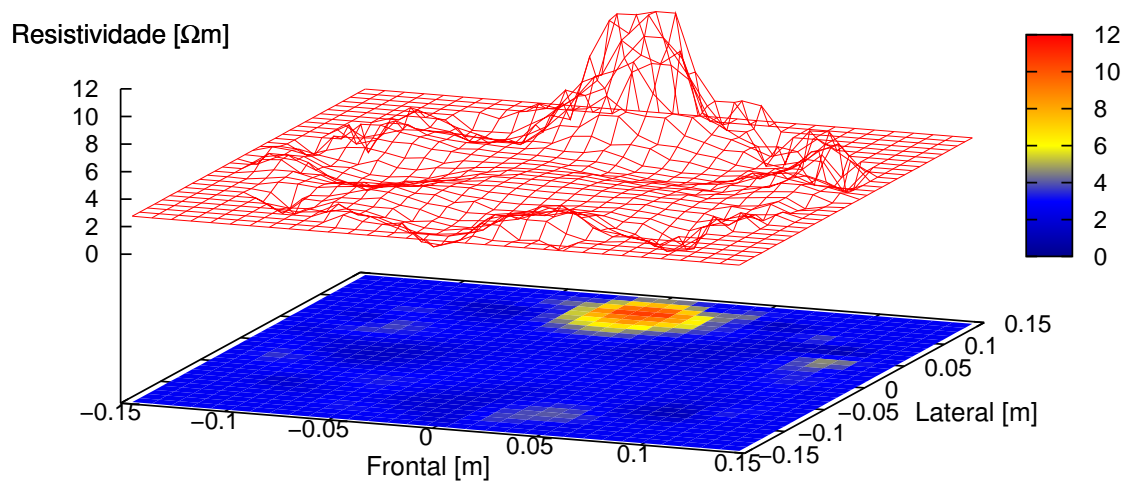

Figura 5.75: Obtenção de resistividade absoluta na borda com dados experimentais usando PLS e RIM para determinar o $\delta \rho$

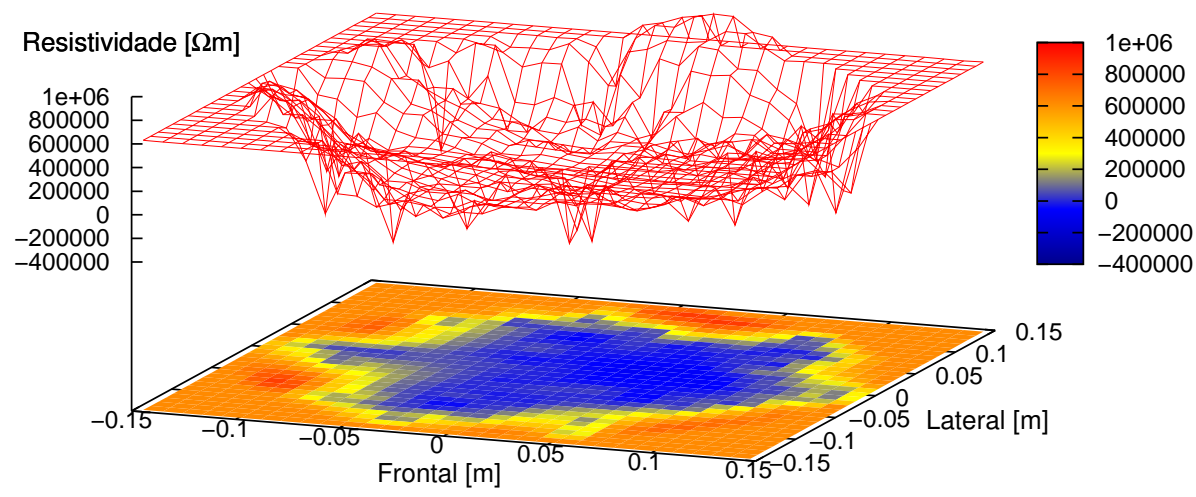

Figura 5.76: Obtenção de resistividade absoluta na borda com dados experimentais usando LU Decomposition para determinar o $\delta \rho$

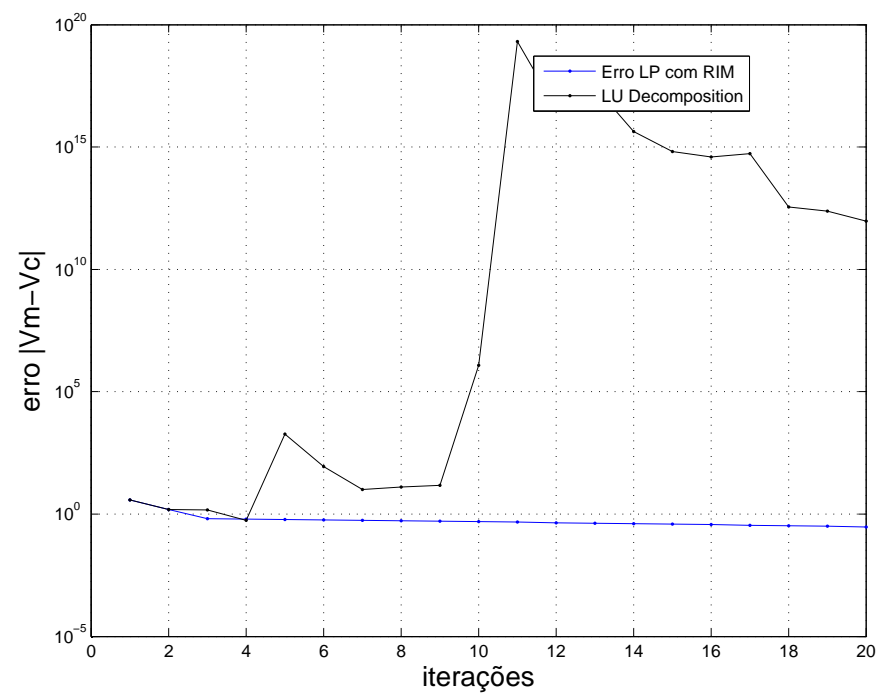

Figura 5.77: Índice de erro entre os potenciais elétricos medidos e os calculados 
- Objeto de acrílico perto do centro Parâmetro $\alpha=1,0 e^{-4}$

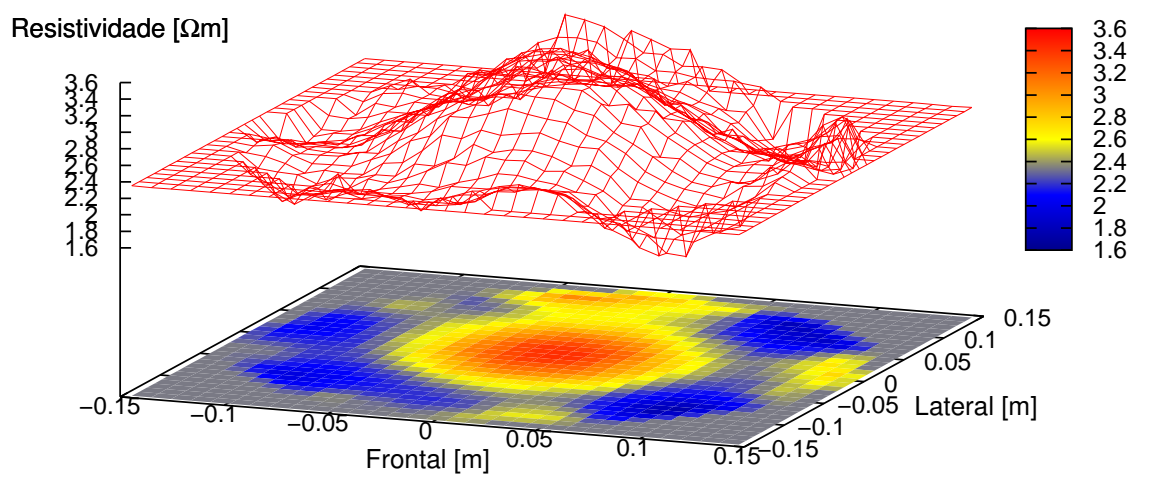

Figura 5.78: Obtenção de resistividade absoluta no centro com dados experimentais usando PLS e RIM para determinar o $\delta \rho$

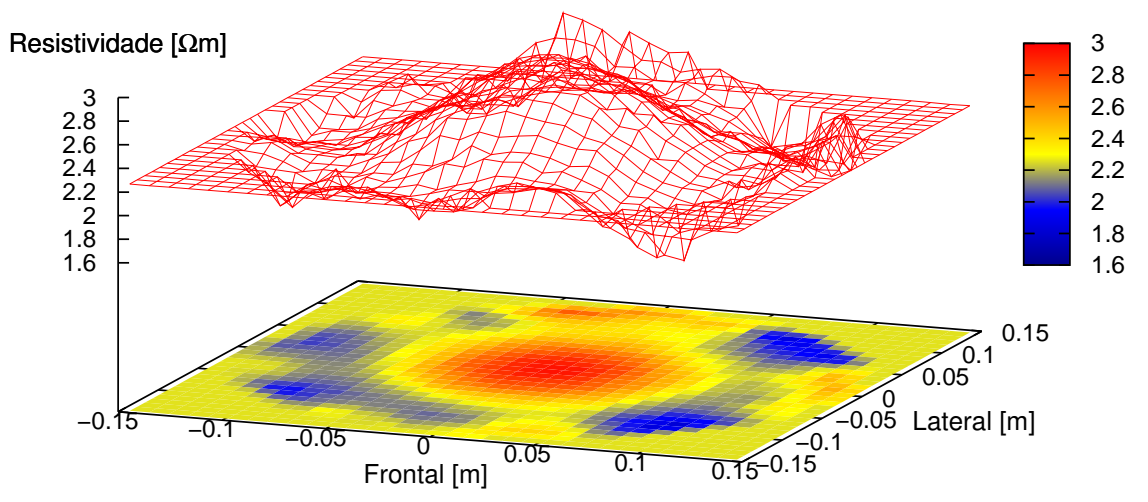

Figura 5.79: Obtenção de resistividade absoluta no centro com dados experimentais usando LU Decomposition para determinar o $\delta \rho$

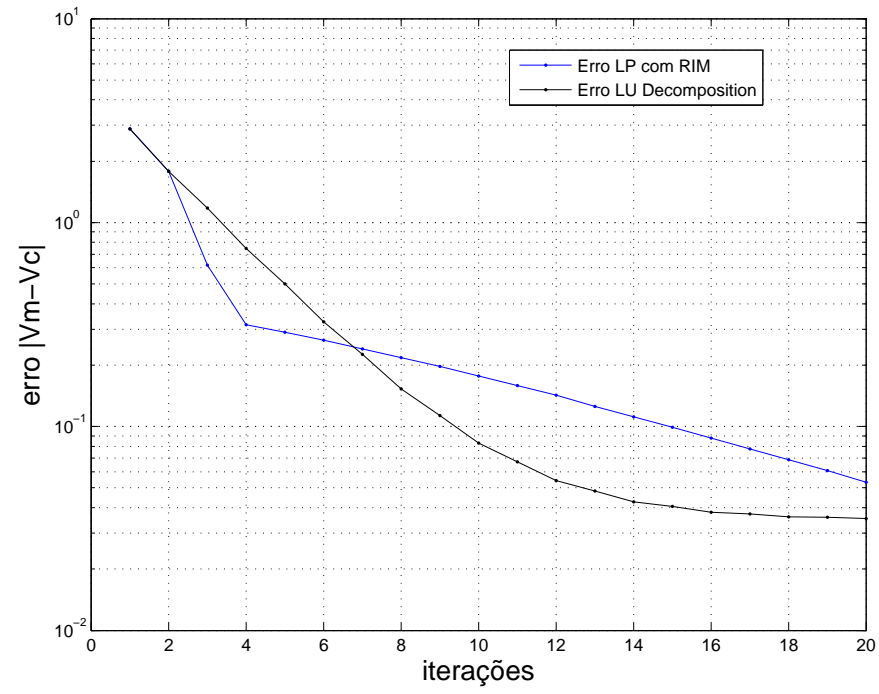

Figura 5.80: Índice de erro entre os potenciais elétricos medidos e os calculados 
- Objeto de acrílico perto do centro Parâmetro $\alpha=\mathbf{1}, 0 e^{-5}$

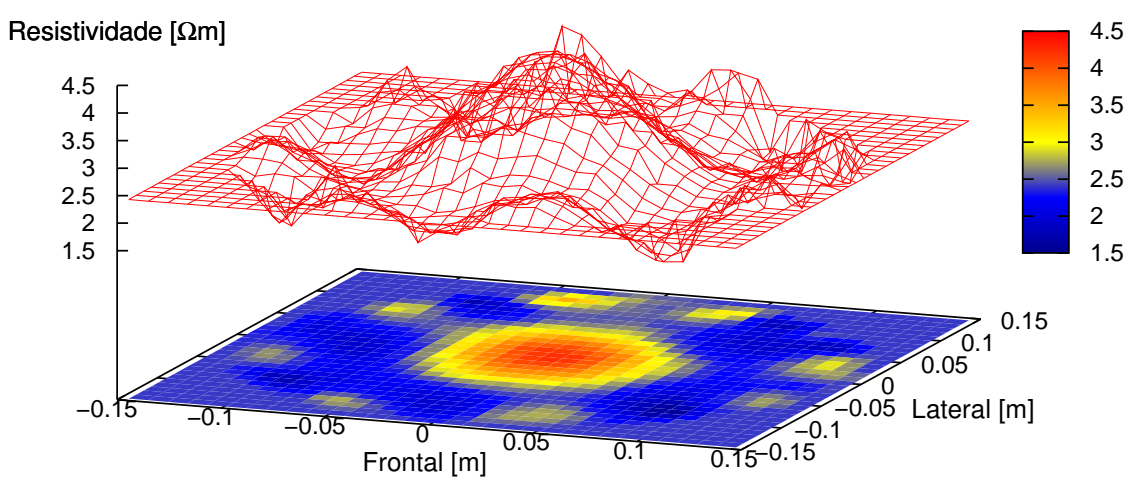

Figura 5.81: Obtenção de resistividade absoluta no centro com dados experimentais usando PLS e RIM para determinar o $\delta \rho$

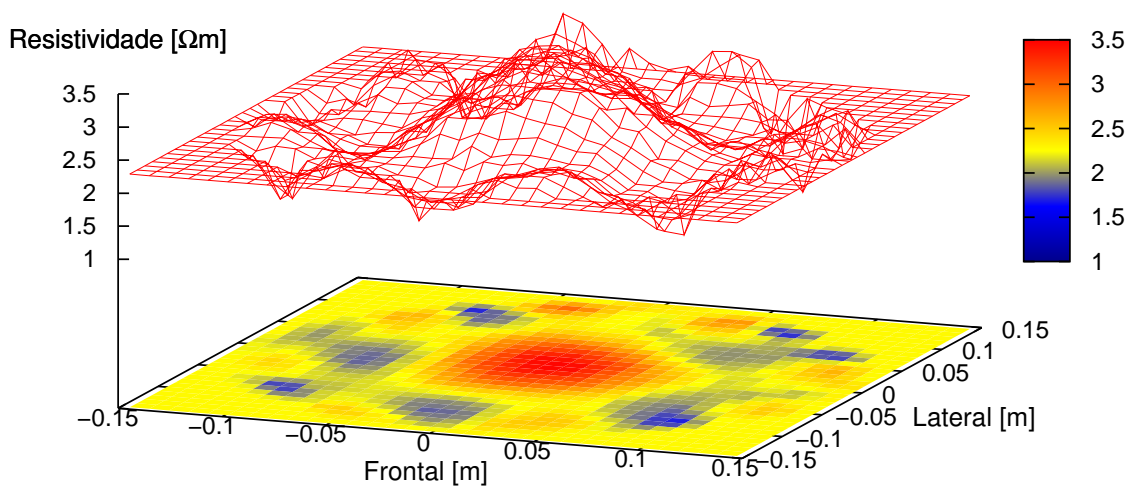

Figura 5.82: Obtenção de resistividade absoluta no centro com dados experimentais usando LU Decomposition para determinar o $\delta \rho$

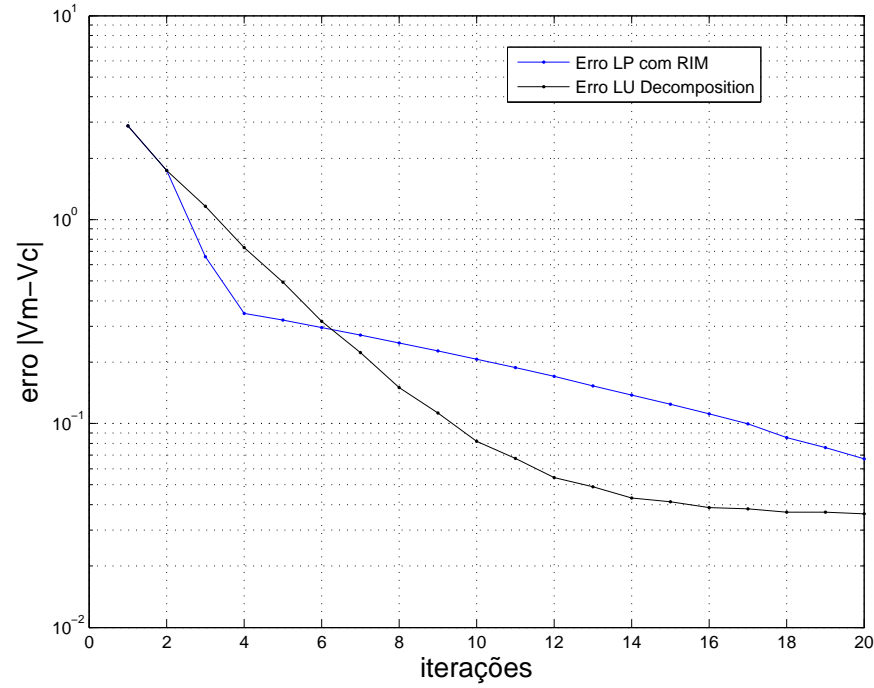

Figura 5.83: Índice de erro entre os potenciais elétricos medidos e os calculados 
- Objeto de acrílico perto do centro Parâmetro $\alpha=\mathbf{1 , 0} e^{-6}$

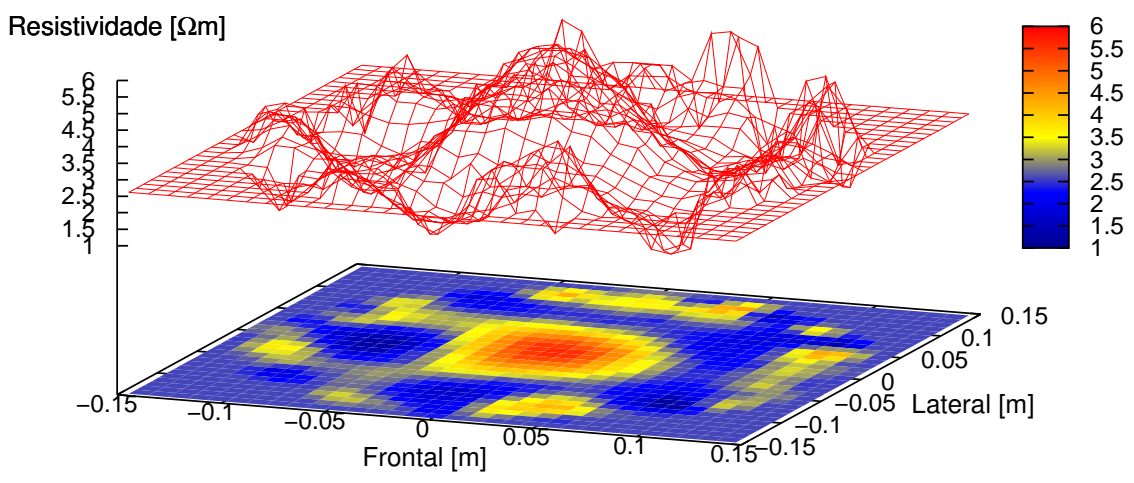

Figura 5.84: Obtenção de resistividade absoluta no centro com dados experimentais usando PLS e RIM para determinar o $\delta \rho$

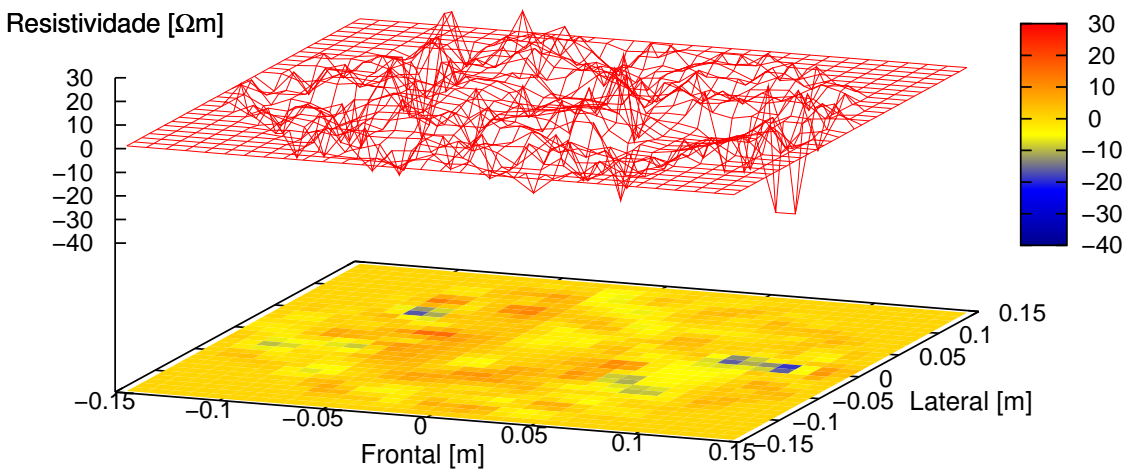

Figura 5.85: Obtenção de resistividade absoluta no centro com dados experimentais usando LU Decomposition para determinar o $\delta \rho$

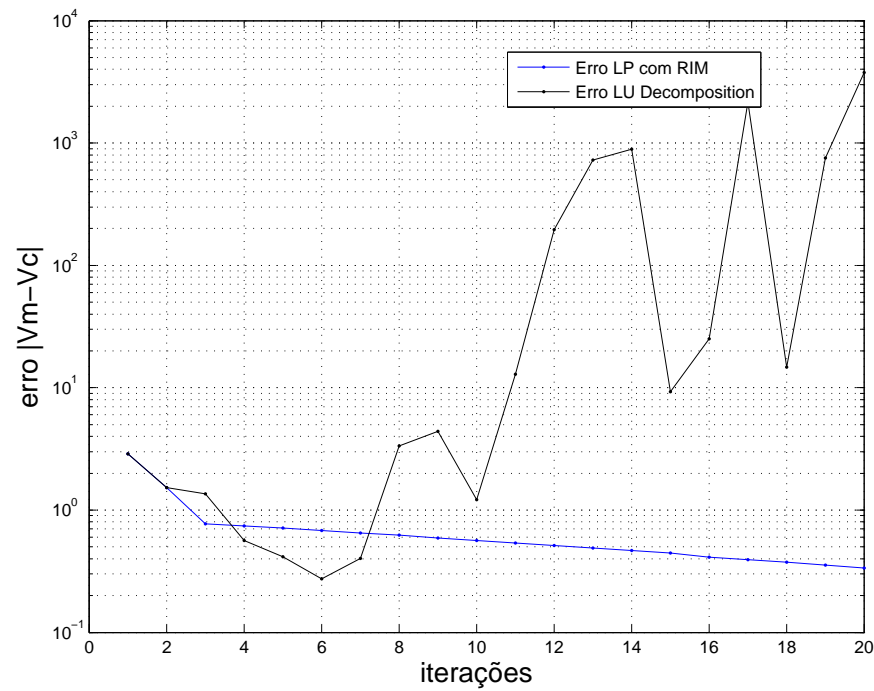

Figura 5.86: Índice de erro entre os potenciais elétricos medidos e os calculados 
- Objetos de agar Parâmetro $\alpha=1,0 e^{-3}$

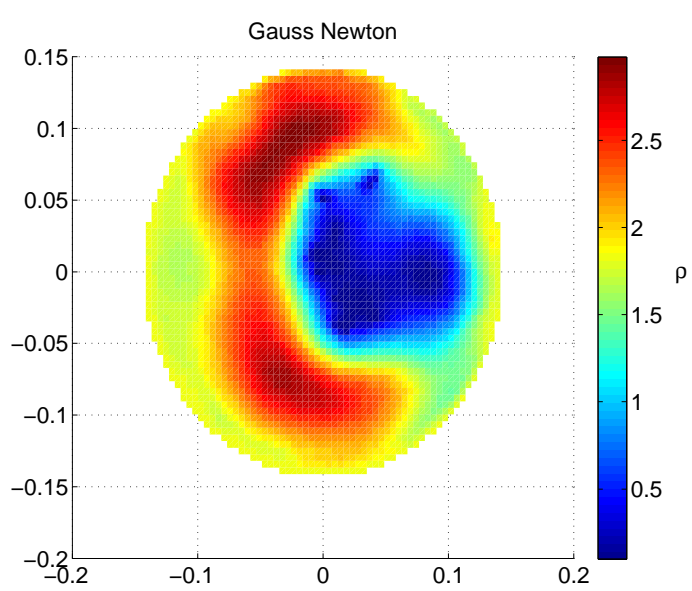

(a)

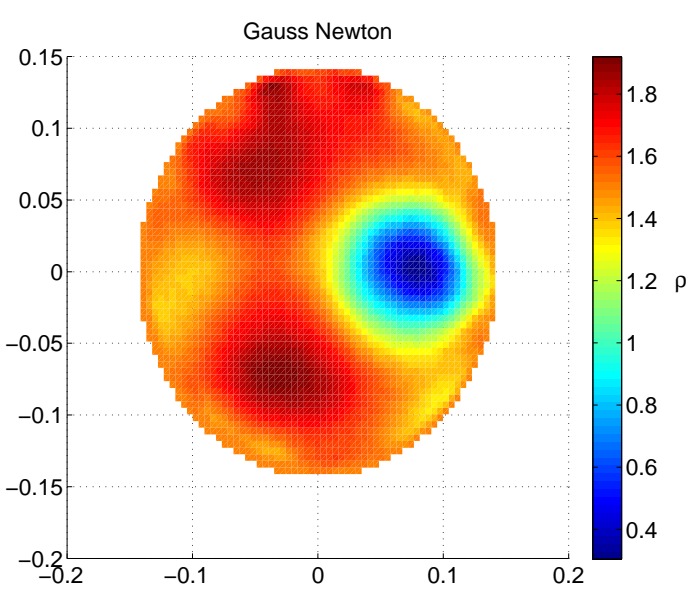

(b)

Figura 5.87: Obtenção de resistividade absoluta com objetos de agar (a) com PLS e (b) LU Decomposition

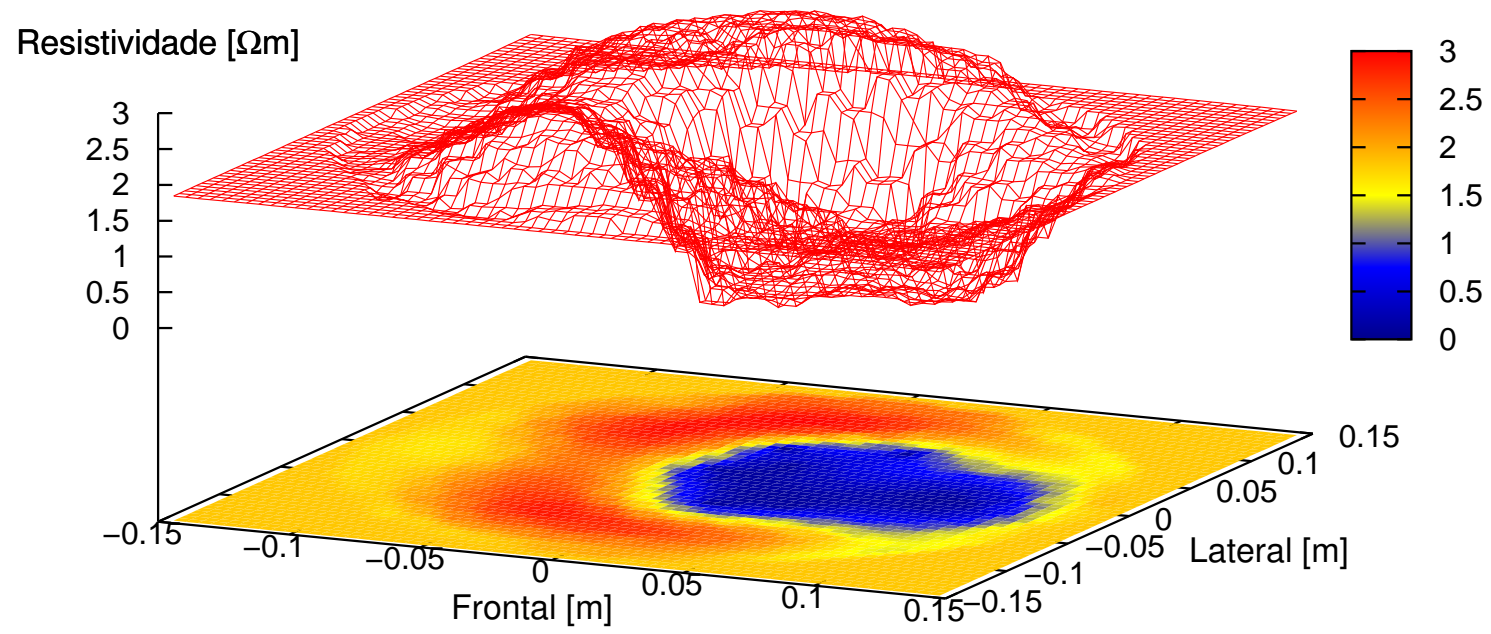

Figura 5.88: Obtenção de resistividade absoluta com objetos de agar usando PLS e RIM para determinar o $\delta \rho$ 


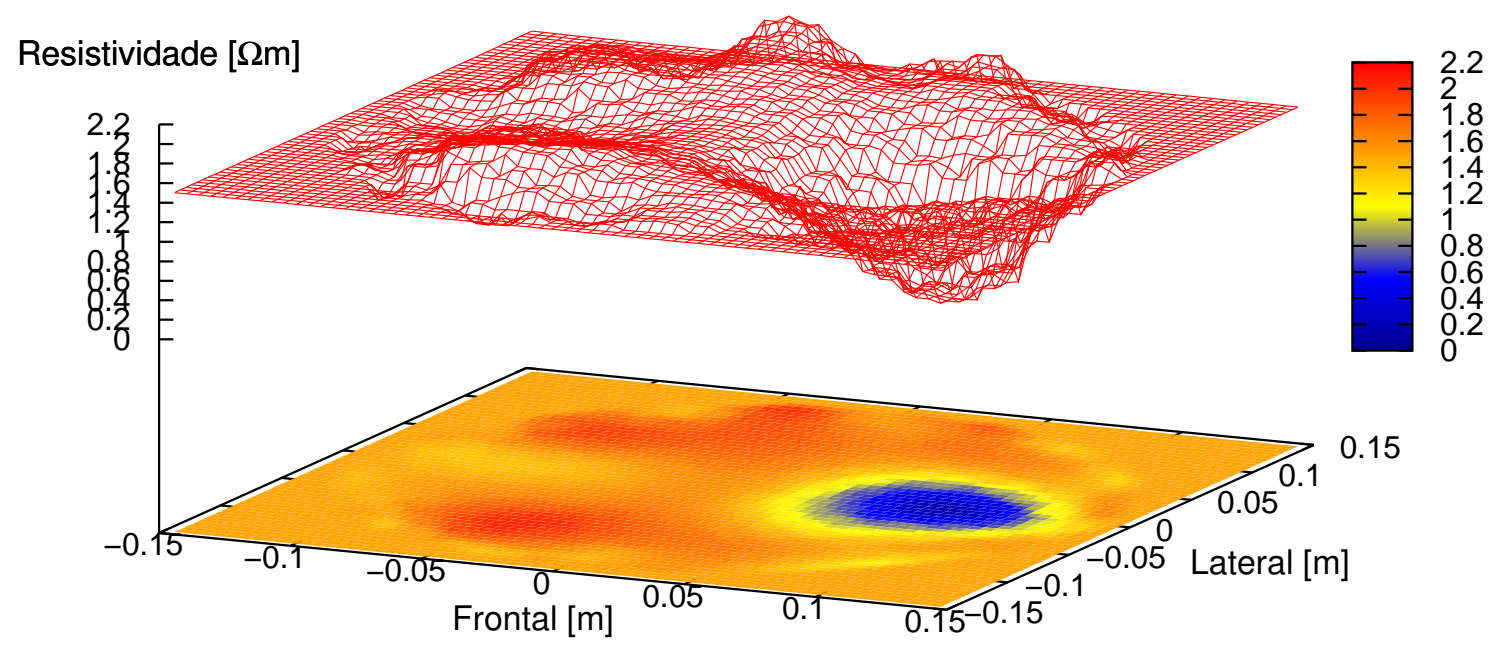

Figura 5.89: Obtenção de resistividade absoluta com objetos de agar usando LU Decomposition para determinar o $\delta \rho$

\subsubsection{Dados do Tórax Humano}

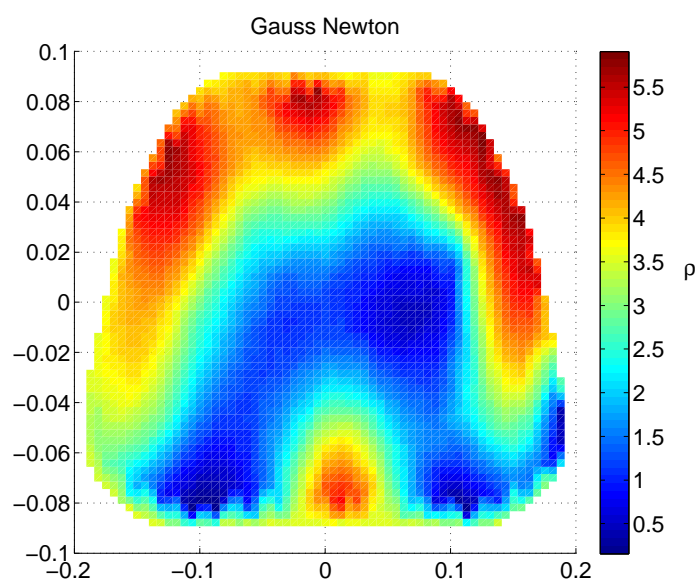

(a)

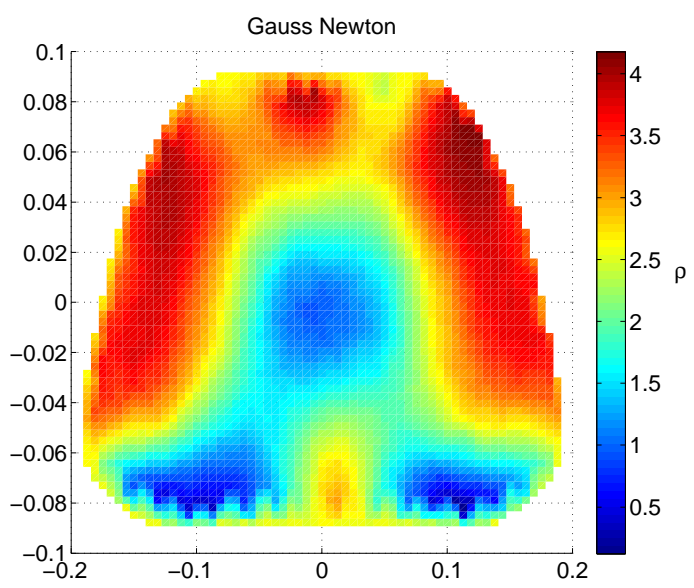

(b)

Figura 5.90: Obtenção de resistividade absoluta no tórax humano (a) com PLS e (b) LU Decomposition 


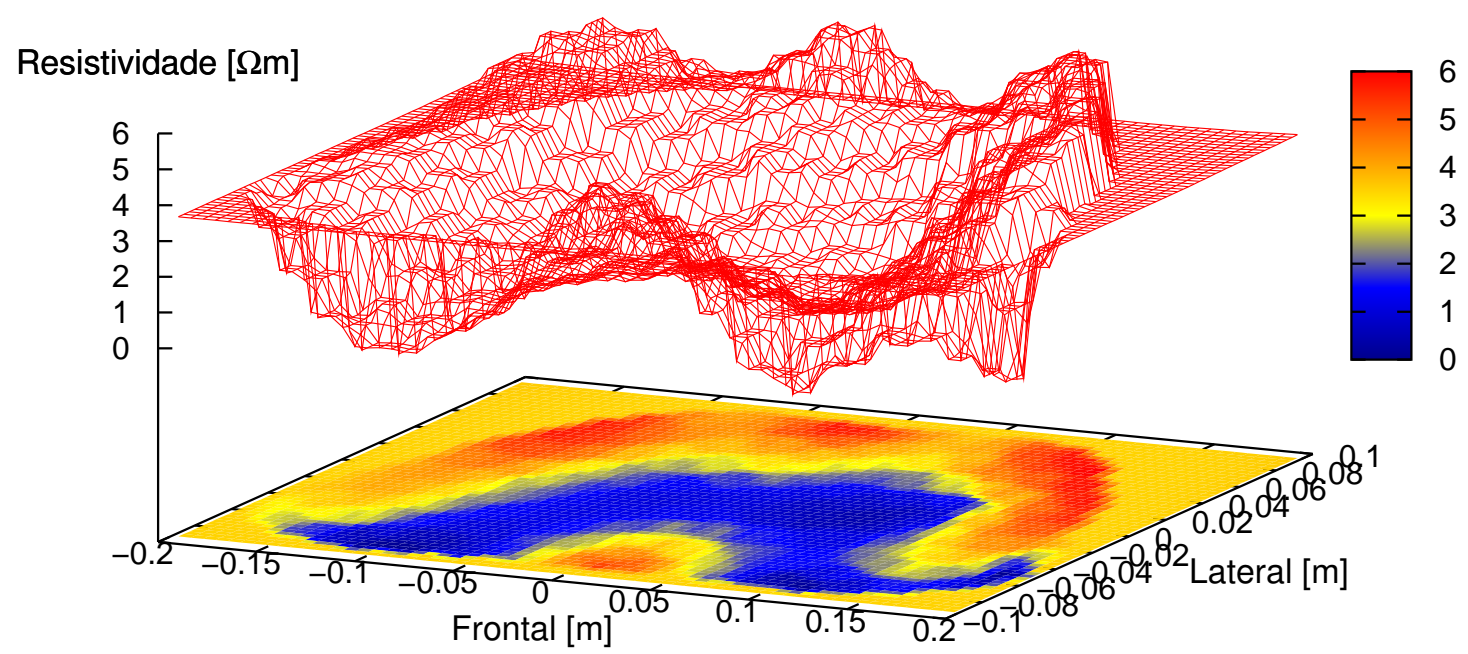

Figura 5.91: Obtenção de resistividade absoluta no tórax humano usando PLS e RIM para determinar o $\delta \rho$

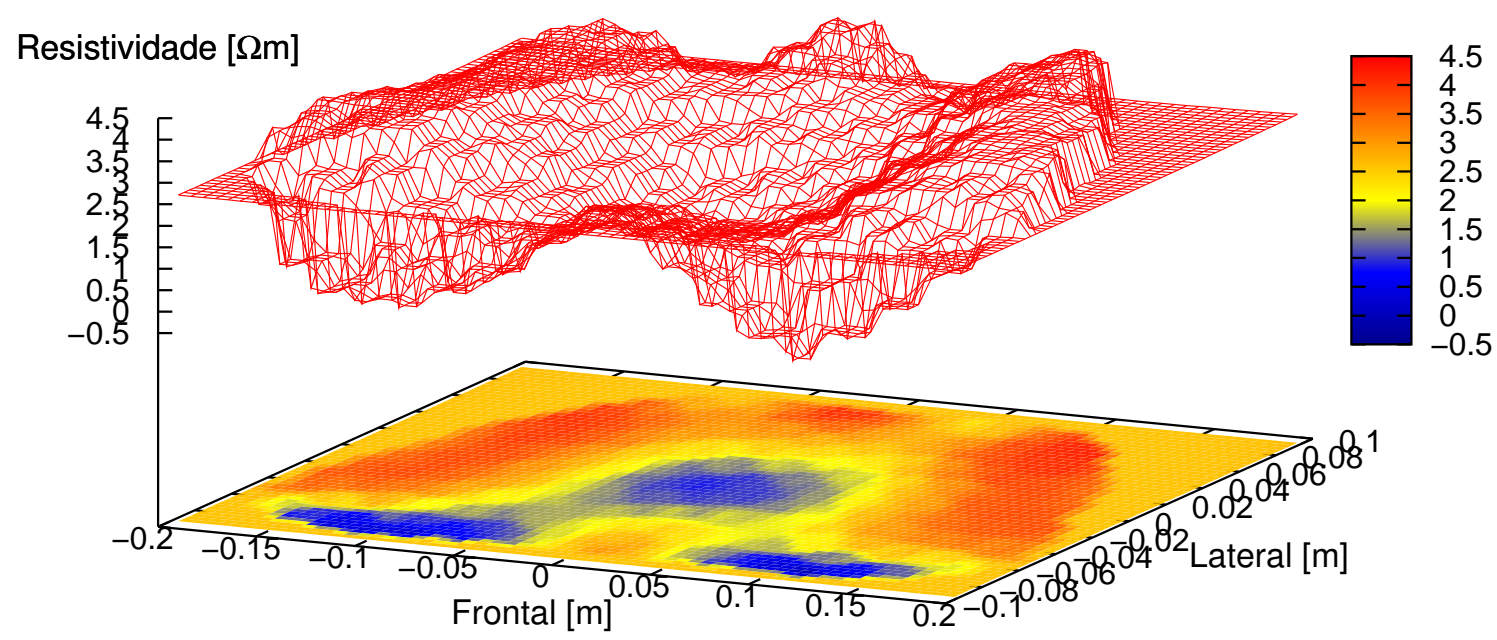

Figura 5.92: Obtenção de resistividade absoluta no tórax humano usando LU Decomposition para determinar o $\delta \rho$ 


\section{$6 \quad$ Análise dos Resultados}

\subsection{PL na Diminuição do Erro Numérico}

Para avaliar a propagação do erro numérico foi utilizado um índice, $\left.\| \mathbf{A A}^{-1}-I\right) \|_{2}$. Foi utilizada a inversa da matriz A obtida com o método LU Decomposition para inicializar a PL e para poder impor restrições nas variáveis, diminuindo o tempo de busca da PL.

Os resultados obtidos na solução do índice quadrático são apresentados nas tabelas 5.1 e 5.2, para diferentes valores dos LS e LI, igualmente para diferentes valores do parâmetro de regularização $\alpha$. Observando os valores ressaltados em cinza, para valores de $\alpha=1,0 e^{-6}$ e $\alpha=1,0 e^{-7}$ o erro na solução do índice quadrático com PL é $18 \%$ menor que a solução obtida com LU Decomposition.

Pode-se destacar que quando o fator de regularização vai diminuindo e as matrizes vão se tornando mal condicionadas, a PL consegue gerar soluções com erro numérico um pouco menor que LU Decomposition. Este resultado é importante uma vez que em TIE o fator de regularização deve ser o menor possível.

\subsection{Comparação dos Resultados Obtidos com PL e LU Decomposition no Problema de Ima- gens de Variação de $\rho$}

\subsubsection{Dados de Simulação Numérica}

Estudando as imagens de dados simulados no problema de obtenção de imagens de variação, observa-se que o método de obtenção de imagens utilizando PL como método de busca mostrou ser mais preciso quando comparado com LU Decomposition em termos de localização do elemento (da malha de elementos finitos) ou do objeto, em termos do tamanho e e em termos do valor da resistividade encontrada. Nestas condições ideais, na qual o ruído nas medidas é nulo, PL permitiu encontrar imagens nas quais a distribuição de resistividade é suave. Observa-se 
que quando o parâmetro de regularização $\alpha$ vai diminuindo tornando mal posta a matriz de sensibilidade, PL conseguiu encontrar alterações de resistividade onde o elemento ou o objeto foi colocado. Nas soluções via LU Decomposition não é possível distinguir alterações de resistividade em nenhuma região do contorno quando alpha vai diminuindo. Isto ilustra a vantagem da PL sobre o LU quanto à propagação de erro numérico.

\subsubsection{Dados da Bancada Experimental}

Observa-se que a localização do objeto nas imagens geradas com PL e LU Decomposition ocorrem no lugar certo. Entretanto a geometria do objeto é mais correta nas imagens via PL. As imagens obtidas via PL também são mais suaves quando comparadas com as imagens obtidas via LU Decomposition. Os valores de resistividade encontrados com PL são maiores quase $50 \%$ do que os encontrados com LU Decomposition. Como no caso anterior, quando o parâmetro de regularização $\alpha$ vai diminuido, as imagens via de PL continuam apresentando distribuições de resistividade acima da resistividade basal em lugares onde o objeto se encontra.

\subsection{Comparação dos Resultados Obtidos com LU Decomposition e PLS sem RIM}

\subsubsection{Dados de Simulação Numérica}

As diferenças de precisão em termos de localização do objeto, tamanho do objeto e precisão na resistividade encontradas são pequenas. Analizando a convergência do método Gauss-Newton, observa-se a convergência do método PLS é mais rápida. Em alguns testes, a convergência do método PLS ocorreu entre a décima e a vigésima iteração, antes que o método LU Decomposition desse sinais de convergência. Neste caso de estudo onde o ruído nas medidas não está presente, observa-se que para um determinado parâmetro de regularização alpha, PLS detecta variação de resistividade na região do objeto sem que o mesmo ocorra no método LU Decomposition. 


\subsection{Comparação dos Resultados Obtidos com LU Decomposition e PLS e RIM}

\subsubsection{Dados de Simulação Numérica}

As imagens obtidas com PLS e RIM são melhores que as imagens obtidas com PLS sem RIM. A convergência do método PLS e RIM mais lenta quando comparada com a convergência do PLS sem RIM. Isto é esperado pois houve um aumento no número de restrições do PLS.

\subsubsection{Dados da Bancada Experimental}

Quando o parâmetro de regularização $\alpha$ diminue há um valor no qual não é possível gerar imagem com LU Decomposition e com PLS é ainda possível encontrar uma imagem não muito boa mas onde é possível observar variação de resistividade na região onde está localizado o objeto.

As imagens obtidas a respeito da bancada experimental com objeto de acrílico identificaram o objeto com valores de resistividade bem subestimados em relação a aqueles esperados pela resistividade do acrílico, $12 \Omega \mathrm{m}$. As imagens obtidas via PLS identificaram valores levemente superiores aos valores identificados via LU Decomposition e muito inferiores ao reportados nos trabalhos sobre otimização topológica, filtros de Kalman e "simulated annealing"aplicados ao problema de TIE. As malhas empregadas aqui são pouco refinadas comparadas às malhas utilizadas nos trabalhos de otimização topológica e isto pode explicar parcialmente esta discrepância.

Entre os dados de bancada experimental, foram adquiridos dados com elementos de agar como mostrado na seção 4.1. O objetivo deste tipo de ensaio, foi avaliar o algoritmo utilizando PLS quando os valores de resistividade são conhecidos a priori. Com a informação da resistividade dos objetos, é possível restringir o espaço solução da PLS com valores mais próximos do modelo físico. A imagem obtida apresentou boa localização dos objetos, pulmões e coração. O tamanho do objeto que representa o pulmão foi próximo do correto mas, o tamanho do objeto que representa o coração foi um pouco superior ao esperado. A faixa de resistividades na imagem encontra-se dentro da faixa medida durante a preparação dos objetos, objetos representando os pulmões com cerca de $3,8 \Omega \mathrm{m}$ e o objeto representando o coração com resistividade assima de $0,8 \Omega \mathrm{m}$. Comparando as imagens obtidas com PLS e com as imagens obtidas via LU Decomposition observa-se que 
a imagem obtida via PLS define melhor a localização dos objetos, os valores de resistividade estão mais próximos dos valores esperados.

\subsubsection{Dados do Tórax Humano}

Este tipo de ensaio é um pouco mais exigente. A imagem obtida decorre de dados provenientes de um tórax humano, tendo ocorrido em condições próximas daquelas que se espera no ambiente clínico. Os dados adquiridos no tórax estão sujeitos a diferentes variáveis entre as quais tem-se o ruído nas medições, a localização dos eletrodos ao redor do tórax e a impedância de contato entre o eletrodo e a pele, as quais podem afetar a imagem obtida pelo algoritmo. Os resultados obtidos com PLS revelam uma imagem em seção transversal onde é possível localizar os pulmões e uma parte da coluna vertebral do individuo, os órgãos observados nas imagens tem uma localização anatômicamente plausível. Os valores de resistividade encontrados estão dentro da faixa dos valores esperados ${ }^{1}$ (ZHANG; PATTERSON, 2006).

O custo computacional utilizando o método Gauss-Newton (com PLS e RIM) mostrou-se elevado para dados com objeto de acrílico, em torno de quase 2 horas para estimar a imagem. Para dados de agar o tempo computacional foi de aproximadamente 7 minutos, diminuindo significativamente mas sendo elevado quando comparado com o tempo que demora resolver o mesmo problema utilizando LU Decomposition que não ultrapassa 40 segundos. No teste com dados do tórax humano, o tempo para gerar imagens utilizando PLS foi de aproximadamente 50 segundos sendo o dobro do tempo que demora LU Decomposition. Cada teste utilizou uma malha de elementos finitos diferente, o que afeta diretamente o tempo computacional de cada simulação. As simulações foram feitas num computador Dell com porcessador intel pentium 4 de $2,8 \mathrm{GHz}$.

\footnotetext{
${ }^{1}$ http://niremf.ifac.cnr.it/tissprop/htmlclie/htmlclie.htm
} 


\section{Comentários Finais}

Foi desenvolvido um algoritmo de Tomografia por Impedância Elétrica baseado em Programação Linear como método de busca da imagem. O algoritmo desenvolvido herda uma das grandes vantagens da Programação Linear que é a diminuição da propagação de erro numérico e a facilidade de restringir o espaço solução diretamente através de restrições de programação linear.

O espaço solução do problema inverso mal-condicionado foi restrito de três maneiras distintas. Inicialmente utilizou-se uma restrição tipo caixa. Para penalizar altas freqüências espaciais na imagem foi implementada uma regularização generalizada de Tikhonov com um filtro espacial passa-alta Gaussiano. E finalmente, implementou-se uma restrição na inclinação máxima no gráfico de resistividade. Através destes exemplos de restrições e regularizações fica claro que o método admite a maioria das regularizações normalmente utilizadas em TIE que tem a forma de regularizações generalizadas de Tikhonov.

Os resultados mostram que implementar PL e PLS na solução de sistemas lineares diminui a propagação de erro numérico, melhora a resolução na resistividade, melhora a resolução espacial e é possível diminuir o parâmetro de regularização quando comparado ao emprego de LU Decomposition na solução de sistemas lineares. Estes fatos são observados tanto nas imagens de diferenças de resistividade quanto nas imagens absolutas.

Com dados de tórax humano o algoritmo de busca de imagem baseado em PLS encontrou os pulmões e a coluna vertebral do indivíduo em estudo com valores de resistividade muito próximos do valor encontrado na literatura, apresentando estes orgãos deslocados para a periferia da seção transversal. A imagem obtida via LU Decomposition também apresenta valores próximos aos encontrados na literatura porém valores mais baixos que os obtidos via PLS. Os orgãos da imagem obtida via LU Decomposition também tem os orgãos deslocados para a periferia da seção transversal. Estes deslocamentos sugerem que a sensibilidade não está uniforme dentro do domínio. 
Recomenda-se para trabalhos futuros implementar uma regularização baseada em um Atlas Anatômico, utilizar modelo 3D do domínio, posicionar os eletrodos fora de um único plano e uma comparação mais detalhada do PLS com RIM com o algoritmo baseado na Otimização Topológica. 


\section{Referências}

ARUCA, D. Algoritmo de tomografia por impedância elétrica baseado em Newton-Raphson. Dissertação (Mestrado) — Dep. de Eng. Mecánica, Escola Politécnica. Universidade De São Paulo., 2002.

ASFAW, Y. W. Automatic detection of detached and erroneous electrodes in Electrical Impedance Tomography. Dissertação (Mestrado) - University of Ottawa, 2005.

BARBER, D.; BROWN, B. Applied potential tomography. Journal of Physics E:Scientific Instruments, v. 17, p. 723-733, 1984.

BELEGUNDU, A. D.; ARORA, J. S. A study of mathematical programming methods for structural optimization. International Journal of Numerical Methods in Engineering, 1985.

BORSIC, A.; MCLEOD, C.; LIONHEART, W.; KERROUCHE, N. Relastic 2d human thorax modelling for eit. Physiol. Meas., 2000.

BREGAlDA, F. P.; OliveirA, A. F.; BORNSTEIN, T. C. Introdução a Programação Linear. Ltda, editora campus. [S.l.]: Ltda, Editora Campus, 1983.

BRYSON, A. E.; HO, Y. C. Applied Optimal Control. Hemisphere publishing corporation. [S.1.]: Hemisphere Publishing Corporation, 1966.

CARDOSO, E. L. Controle de complexidade na otimização topológica de estruturas contínuas. Dissertação (Mestrado) - Escola de Engenharia da Universidade Federal do Rio Grande do Sul, 2000.

CHARNES, A.; COOPER, W. W.; MELLON, B. Blending aviation gasolines a study in programming interdependent activities in an integrated oil company. Econometrica, 1952.

CHENEY, M.; ISAACSON, D.; NEWELL, J. C. Electrical impedance tomography. Society for Industrial and Applied Mathematics, 1999.

CHURCH, P. M.; WORTY, P. M.; GAGNON, S.; MCFEE, J. Performance assessment of an electrical impedance tomography detector for mine-like objects. Defence Research Establishment Suffield DRES.

CITRON, S. J. Elements of Optimal Control. Holt, rinehart and winston. [S.l.]: Holt, Rinehart and Winston, 1969.

DOBSON, D. Convergence of a reconstruction method for the inverse conductivity problem. SIAM Journal on Applied Mathematics, v. 52(2), p. 442-458, 1992. 
EGGLESTON, M.; SCHWABE, R.; ISAACSON, D.; COFFIN, L. The application of electric current computed tomography to defect imaging in metals. [S.l.: s.n.], 1989.

FABIAN; TIBOR. Process type analysis of the iron and steel industry, part iv-programming open hearth steel production. Management Sciences Research Project, University of California, 1954.

FEHMERS, G. Volumetric flow rates from impedance tomography in oil/gas flows. 3rd World Congress on Industrial Process Tomography, 2003.

GEORGE, D. L.; TORCZYNSKI, J. R.; SHOLLENBERGER, K. A.; OHERN, T. J.; CECCIO, S. L. Validation of electrical-impedance tomography for measurements of material distribution in two-phase flows. International Journal of Multiphase Flow, 2000.

GILL, P. E.; MURRAY, W.; WRIGHT, M. H. Practical Optimization. Academic press. [S.l.]: Elsevier Science, 1986.

GOnZAlez, R. C.; WOODS, R. E. Digital Image Processing. Prentice-hall. [S.1.]: Tom Robinns, 2002.

HENDERSON, A.; SCHLAIFER, R. Mathematical programming. Harvard Business, 1954.

HUA, J. W. P.; WEBSTER, J.; TOMPKINS, W. Finite element modeling of electrode-skin contact impedance in electrical impedance tomography. IEEE Transactions on Biomedical Engineering, v. 40, n. 4, p. 335-343, 1993.

KAIPIO, J.; KOLEHMAINEN, V.; SOMERSALO, E.; VAUHKONEN, M. Statistical inversion and monte carlo sampling methods in electrical impedance tomography. Inverse Problems, v. 16, n. 5, p. 1487-1522, 2000.

LELLEP, J.; MAJAK, J. Optimal material orientation of nonlinear orthotropic materials. Mechanics of Composite Materials, 1999.

LIMA, C.; SILVA, E.; LIMA, R. Topology optimization method applied to obtain images from electrical impedance tomography technique in: 17th international conference of mechanical engineering, 2003, s?o paulo. Proceedings of the 17th International Conference of Mechanical Engineering, p. 535-538, 2003.

LIMA, C. R. de. Estudo da Obtenção de Imagens de tomografia de Impedância Elétrica do Pulmão pelo Método de Otimização Topológica. Tese (Doutorado) Escola Politécnica da Universidade de São Paulo, 2006.

LOGAN, D. L. A First Course in the Finite Element Method. Bil stenquist. [S.1.]: Thomson Brooks/Cole, 2002.

MAKHORIN, A. GNU Linear Programming Kit. GNU Project, 2004. Disponível em: <http://www.gnu.org/>.

MANGASARIAN, O. L.; STREET, W. N.; WOLBERG, W. H. Breast cancer diagnosis and prognosis via linear programming. Operations Research, 1995. 
MELLO, L. A. M. Estudo da Obtenção de imagens tridimensionais de Tomografia por Impedância Elétrica pelo Método de Otimização Topológica. Dissertação (Mestrado) — Escola Politécnica da Universidad de São Paulo, 2005 .

MURPHY, D.; BURTON, P.; COOMBS, R.; TARASSENKO, L.; ROLFE, P. Impedance imaging in the newborn. Clin. Phys. Physiol. Meas, 1987.

MYER, D.; CONSTABLE, S.; KEY, K. Electrical Impedance Tomography of a Seafloor Volcano. [S.l.].

POURAZADY, M.; FU, Z. An integrated approach to structural shape optimization. Computers \& Structures, 1996.

PRADO, D. Programação Linear. [S.l.]: DG, 1999.

ROSELL, J.; COLOMINAS, J.; RIO, P.; PALLAS-ARENY, R.; WEBSTER, J. Skin impedance from $1 \mathrm{hz}$ to $1 \mathrm{mhz}$. IEEE Transactions on Biomedical Engineering, BME-35, p. 649-651, 1998.

SADIKU, N. Numerical Techniques in Electromagnetics. [S.l.]: Boca Raton, 1992.

SANTOSA, F.; VOGELIUS, M. Backprojection algorithm for electrical impedance imaging. SIAM Journal on Applied Mathematics, v. 50, p. 216-243, 1990 .

STRATTON, J. A. Electromagnetic Theory. Mcgraw-hill. [S.l.]: McGraw-Hill, 1940 .

TIDSWELL, T.; GIBSON, A.; BAYFORD, R. H.; HOLDER, D. S. Threedimensional electrical impedance tomography of human brain activity. Neuroimage, 2001.

TRIGO, F. Filtro estendido de Kalman aplicado à tomografia por impedância elétrica. Dissertação (Mestrado) — Dep. de Eng. Mecânica, Escola Politécnica. Universidade De São Paulo., 2001.

TRIGO, F. C. Estimação não Linear de Parâmetros através dos Filtros de Kalman na Tomografia por Impedância Elétrica. Tese (Doutorado) - Escola Politécnica da Universidade de São Paulo, 2005.

VANEGAS, N. Propagação de erro numérico no Filtro estendido de Kalman aplicado à tomografia por impedância elétrica. Dissertação (Mestrado) - Dep. de Eng. Mecânica, Escola Politécnica. Universidade De São Paulo., 2002.

VAUHKONEN, M.; KARJALAINEN, P.; KAIPIO, J. Kalman filter approach to track fast impedance changes in electrical impedance tomography. IEEE Transactions on Biomedical Engineering, v. 45, n. 4, p. 486-493, 1998.

VAUHKONEN, M.; KARJALAINEN, P. A.; KAIPIO, J. P. A kalman filter approach to traxk fast impedance changes in electrical impedance tomography. IEEE Transactions on Biomedical Engineering, 1998.

WATERFALL, R. C.; HE, R.; WhitE, N. B.; BECK, C. M. Combustion imaging from electrical impedance measurements. Meas. Sci. Technol, 1996. 
WILliAMS, R. A.; DICKIN, F. J.; GUTIERREZ, J. A.; DYAKOWSKI, T.; BECK, M. S. Using electrical impedance tomography for controlling hydrocyclone underflow discharge. 1997.

WOO, E. J.; HUA, P.; WEBSTER, J. G.; TOMPKINS, W. J. Measuring lung resistivity using electrical impedance tomography. IEEE TRANSACTIONS ON BIOMEDICAL ENGINEERING, 1992.

YORKEY, T.; WEBSTER, J.; TOMPKINS, W. Comparing reconstruction algorithms for electrical impedance tomography. Transactions on Biomedical Engineering, v. 34, n. 11, p. 843-852, 1987.

ZHANG, J.; PATTERSON, R. P. Eit images of ventilation: what contributes to the resistivity changes ? Institute of Physics Publishing, 2006. 